\title{
Neoanalisi e intertestualità dell'Inno omerico ad Apollo: modelli, immagini, stemma
}

Eleonora Colangelo

(Paris Diderot University, Centre ANHIMA, University of Pisa)

\section{Neoanalysis and Intertextuality in the Homeric Hymn to Apollo: Models, Motifs, and Stemma}

\begin{abstract}
The aim of this paper is to illustrate a first neoanalytical frame of the Homeric Hymn to Apollo $(H H A)$ intertextuality in function of a new critical edition with commentary. After Currie 2011's reflections about neoanalysis for the archaic hymns to Demeter, and the hermeneutical models of Olson 2012 and Vergados 2013 for the Homeric Hymns to Aphrodite and Hermes, I will show and discuss a provisional stemma of the genetic relationships between the HHA and a limited sample of honorific-dedicatory, cathartic-programmatic, and performative-hymnodic texts. The whole scheme of relationships has been established with regard to the productivity and recurrence of seven re-semantizated topoi. The stemma will enable us to define the domain of HHA prehistory, survivals and reminiscences in literary and paraliterary production before and after the terminus ante quem of $478 \mathrm{BC}$.
\end{abstract}

\section{Keywords}

intertextuality; neoanalysis; Homeric hymn to Apollo; intertextual stemma; papyri; Delphic hymns 


\section{Gli Inni omerici e i transferred motifs della neoanalisi: un'introduzione}

La nozione di literary history ha conosciuto un'applicazione alquanto controversa all'ambito della poesia esametrica arcaica. Un contributo fondamentale sulla questione della cronologia relativa, strettamente connessa a quella della literary history, è stato dato da B. Currie tramite l'analisi in chiave neoanalitica delle relazioni letterarie tra vari inni arcaici a Demetra. ${ }^{1}$ Mirando ad una riflessione più generale sulla possibile esistenza di discrete works, o fixed texts, nella tradizione d'origine orale, Currie muove dalla seguente assunzione: se un testo $x$ può dimostrarsi alludere ad un testo $y, y$ è di più alta datazione rispetto ad $x$. Solo negli ultimi anni la sfida di determinare l'esistenza, in un dato testo, di echeggianti allusioni ad un altro è stata ampiamente accolta dagli studiosi. ${ }^{2}$ Ciò è stato possibile dando diverse interpretazioni del concetto stesso di intertestualità rispetto a quello di narratologia, e proponendo diversi adattamenti dell'indagine intertestuale secondo i criteri della mythological intertextuality (possibile grazie alla conoscenza condivisa di uno stesso repertorio tradizionale di miti da cui attingere $)^{3}$ e della traditional referentiality (conseguenza, questa, del gioco operato dal poeta su motivi e formule tradizionali). ${ }^{4}$

Ciò premesso, l'obiettivo di questo articolo sarà fornire un primo profilo dell'identità letteraria dell'Inno omerico ad Apollo ( $h A p$. d'ora in avanti), testo che concentra in piccolo elementi e costanti da sempre cari alla critica omerica. ${ }^{5}$ Nel presente contributo si considererà un insieme di testi trasmessi per via prevalentemente papiracea ed epigrafica. Di ogni autore verranno quindi tralasciati testi conosciuti per il tramite della tradizione diretta o indiretta, i quali porrebbero di fatto ulteriori questioni di ordine metodologico. Gli stessi dovranno pertanto essere oggetto in futuro di un'indagine specifica. È inoltre opportuno precisare che i testi selezionati non rappresentano che singoli punti di una più complessa trasmissione testuale, e che dunque i dati che emergerrano dalla loro lettura necessiteranno di un'analisi circostanziale. Dividendo il contributo in due sezioni, dedicate la prima alla tradizione papiracea, la seconda alla tradizione epigrafica, si cercherà di capire se un'inter-testualità possa essere presupposta e ricostruita per l'hAp. e in generale l'inno inteso come super-genere letterario. ${ }^{6}$ Nel dettaglio, si procederà al reperimento delle sopravvivenze dell' $h A p$. nella produzione anteriore, coeva e successiva al 478 a.C. (terminus ante quem che ho altrove proposto e disucsso in merito alla cronologia

1 Currie (2011). Per il problema della cronologia relativa della poesia epica omerica, restano ad oggi imprescindibili le note di West (1995).

2 Dopo Kullmann (1984), si vedano Clark (1986), Bonanno (1990), Currie (2011), Montanari \& Rengakos \& Tsagalis (2012), con Martin (2013). Si veda inoltre Burgess (2006: pp. 152-162), per un confronto tra la neoanalisi e l'indagine tipologica. Più di recente, Fantuzzi \& Tsagalis (2015). Per l'approccio narratologico, Faulkner \& Hodkinson (2015).

3 Burgess (2006: p. 173).

4 Foley (1991), Graziosi \& Haubold (2005).

5 Così per Burkert (1979: p. 53).

6 Nagy (2011: p. 333) che propone la definizione di “super-genre” attingendola da Martin (2005) per l'epica. 
relativa dell' $h A p.) .{ }^{7}$ Di queste sopravvivenze, si proporrà una ricostruzione nello stemma provvisorio posto a chiusura del contributo.

1. L'hAp. nei papiri: P. Herc. 1088, P. Lit.Goodspeed 2, PMGF 222b, P. Oxy. 841, P. Oxy. $2240+2442$, P. Oxy. 1792

A differenza dell'hDem. e dell'hDion. (I), la trasmissione dell'hAp. ci è garantita da soli manoscritti. ${ }^{8}$ Pertanto, ciò che si proporrà qui di indagare non è tanto l'apporto dei papiri rispetto alla forma attuale del testo, quanto il grado di allusione, adattamento e persistenza dell' $h A p$. nella tradizione (prevalentemente) letteraria su papiro. ${ }^{9} \mathrm{~A}$ tal fine, integrerò alla lista di esemplari più noti, come P.Oxy. 2440, altri sinora omessi per ragioni legate non da ultimo al loro stato di conservazione. Le differenti versioni del mito saranno valutate in base al mutare delle forme discorsive imposte dal contesto di esecuzione. Variando nella sua veste enunciativa, ogni singolo fotogramma attinto dall' $h A p$. non può infatti non dirsi carico, nelle sue continuità o fratture, di significati metaforici intellegibili se non in relazione allo spazio cultuale condiviso al momento della performance. ${ }^{10}$ Una parte importante del contributo verterà dunque sul trasferimento di immagini in contesti enunciativi pragmaticamente differenti da quelli della supposta $\pi \alpha v \eta ́ \gamma v$ ๖ı ionica che, per prima, avrebbe accolto la performance dell' $h A p .{ }^{11}$ Nella sezione che segue si terrà parimenti conto della distribuzione non uniforme dell'epiteto nell' $h A p$. nonché della sua fortuna nei papiri letterari. ${ }^{12}$

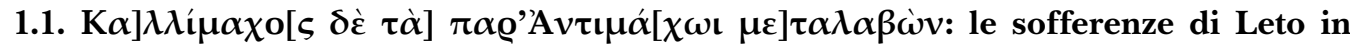 P.Herc. 1088 IIb + 433 II ( = p. 93 Schober $=$ fr. $78 S H=94$ Matthews $)$}

Partire dal P.Herc. 1088 permette di introdurre la fortuna dell' $h A p$. in epoca ellenistica senza passare per la già abbondamente esplorata questione del debito callimacheo. Il papiro in questione trasmette un passaggio attribuibile alla seconda parte del De pietate di Filodemo. Incentrato sulle fatiche degli dèi, questo trattato testimonia in nuce la trasformazione del parto di Leto in uno dei paradigmi rappresentativi della sofferenza divina. ${ }^{13}$

7 Colangelo (2016).

8 Per l'hDem. si veda P.Berl. 13044 (Berliner Klassikertexte V I, 1907, n. 44) e P.Oxy. 2379; per l'hDion., P.Gen. inv. 432 (118 e 121), per la cui edizione si rinvia a Schubert (1996: pp. 15-20).

9 Si pensi alla maggior fortuna dell'hAphr. nei papiri, per cui Barbantani (2005).

10 Calame (2015: pp. 15-20). Si veda ancor prima Vernant (1974: pp. 217-250). Tra i contributi sul mythos (cfr. Vernant 1962: pp. 107 e ss.) che hanno tentato di ridurre la mitologia non alla semplice nozione moderna di mito, si ricordano qui Gantz (2004) e Bierl \& Lämmle \& Wesselmann (2007). Per una riflessione precedente a questi, rinvio a Detienne (1981: pp. 225-242) e Calame (1998: pp. 127-149). Per un'attualizzazione dell'asse mito-rituale-enunciazione, Calame \& Ellinger (2016).

11 hAp. 147-152.

12 Si veda la messa a punto sull'epiteto in Parker (2003: pp. 173-183), con dei precedenti in Detienne (1988-1989: pp. 267-270).

13 Su Filodemo e le fonti d'erudizione, Arrighetti (2003: pp. 13-30). Contra Tarán (1981: p. 450). Un analogo tema da approfondire nella prosa filodemea rispetto alla tradizione innica sarebbe a mio avviso quello della nascita e morte di Dioniso in Euforione di Calcide alla luce di P.Herc. 247 III ( = Orph. fr. 59, I, PEG II). Cfr. [Orph.] frr. 89, 280-283, PEG II; [Orph.] fr. 301-317, PEG II; [Orph.] frr. 321-322, 327, PEG II. Sulla presenza dei poeti ellenistici in P.Herc. 1088, Ciampa (2006). 
Fautore di questo riuso, Antimaco, citato da Filodemo, avrebbe pertanto diversificato in fase pre-alessandrina il tema del travagliato parto di Leto.

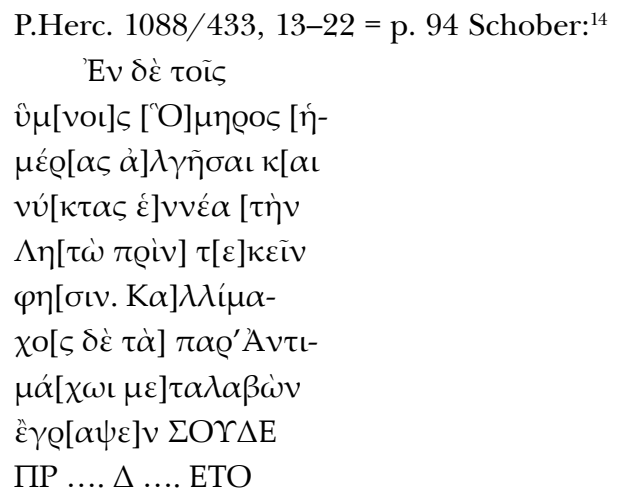

"Negli inni Omero dice che per nove giorni e nove notti Leto soffrì, prima di partorire. Callimaco, riprendendo le notizie fornite da Antimaco, ha scritto che...”. In questo frammento, inizialmente attribuito a Callimaco nell'editio princeps di Gomperz del 1866 (basatasi a sua volta sul solo P.Herc. 433), e poi incluso negli adespota nell'edizione callimachea di Pfeiffer, non saremmo di fronte ad una citazione diretta, come suggerirebbe d'altronde il $\mu \varepsilon] \tau \alpha \lambda \alpha \beta \omega \dot{\omega}$ di Schober. ${ }^{15}$ La restituzione del testo grazie alla ricostruzione di Boserup dei margini delle colonne ha rappresentato in tal senso un decisivo progresso. ${ }^{16}$ Grazie ad essa, infatti, possiamo credere ad oggi che Antimaco sia stato la fonte mitografica - o quantomeno una delle principali - di Callimaco. Non è escluso che Antimaco abbia inoltre elaborato il modello principale dell' $h A p$. per fornirne una versione personale, e che la trasformazione della sofferenza di Leto in paradigma mitico abbia coinciso con la valorizzazione del ruolo di Era nella vicenda del parto. ${ }^{17}$ La presenza di Era nel testo è attualmente leggibile grazie alla felice restituzione di Henrichs nella

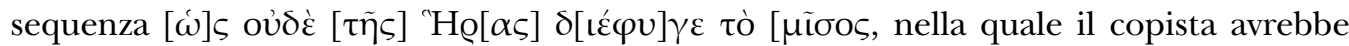
erroneamente trascritto, in $\Pi$ P .... $\Delta$.... ETO (1.31), $\Pi$ al posto di $\mathrm{H}$. In questa versione del mito, non attribuibile nel dato innovativo a Callimaco, l'ira di Era sarebbe dunque diventata la principale fonte di sofferenza di Leto. ${ }^{18}$ D'altra parte, resta ad oggi impossibile stabilire a quale opera di Antimaco Filodemo alludesse, se alla Lyde, in cui l'autore

14 Henrichs (1972: pp. 73-75).

15 Cfr. fr. 92 Matthews = 102 Wyss, in cui leggono Antimaco sia Bernabé (fr. 27 II $P E G$, p. 185) sia Davies (fr. 22 B $E G F$, p. 126). Cfr. Reed (1996: pp. 361-386).

16 Boserup (1971: p. 109).

17 Mineur (1984: p. 9). Per Era nell'hAp., cfr. Forderer (1971: p. 187), in particolare sull'opposizione della dea come "eine olympische Familienintrige"; e ancora, Strauss Clay (1989: p. 49); West (1975: pp. 169-170); Aloni (2004). Si segnala qui la più recente, fine, analisi dell'Era di Zeus in Pironti \& Pirenne Delforge (2016), e in particolare p. 77 n. 217 per Callimaco, "développant le thème de la colère d'Héra (...)".

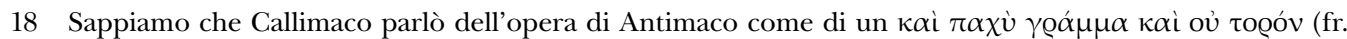
398 Pfeiffer $=715$ A Matthews $=19$ Wyss). Sul rapporto tra Antimaco e Callimaco, si veda Krevans (1993: pp. 150-153). Per Callimaco nel De Pietate, si consideri inoltre anche P.Herc. 243 (p. 101 Schober). 
avrebbe per l'appunto narrato di sofferenze divine dovute al continuo peregrinare,$^{19} \mathrm{o}$ se all'Artemis. ${ }^{20} \mathrm{Il}$ frammento si interrompe laddove esso sembrerebbe spiegare i termini esatti in cui la versione di Antimaco e Callimaco si sarebbe differenziata dal modello principale. Ed è proprio lì che per noi è opportuno fermarsi.

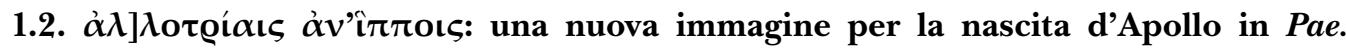 VIIa-b (P. Oxy. 2240 + P. Oxy. 2442)}

Ancor prima di Antimaco, Pindaro rielabora la nascita di Apollo in $h A p$. ponendo l'accento non tanto sulle figure femminili coinvolte nel parto, quanto su quella maschile di Zeus e Delo. ${ }^{21}$ Tre sono i frammenti in cui Pindaro narra della nascita di Apollo a Delo: (a) Pa. VIIb, formato da due sezioni superstiti trasmesse da P.Oxy. 2440 fr. 1 e P.Oxy. 2442 fr. 14, a cui si aggiunge P.Oxy. 841 fr. 17, in cui si menzionano Omero ed una tradizione da cui prendere le distanze cavalcando altre strade, ${ }^{22}$ (b) Pa. XII 33c-d, contenente un'invocazione a Delo all'interno di un più ampio inno (forse) a Zeus. Concepito per un'esecuzione in contesto delio, come suggerito dalla seconda parte del titolo, [AI $\Sigma$ EI $\Sigma$ $\Delta \mathrm{H} \Lambda \mathrm{O}] \mathrm{N}$, il settimo peana consta di due sezioni, l'una programmatica l'altra propriamente narrativa. ${ }^{23}$ Sin dai primi versi Pindaro prende le distanze da una tradizione pre-

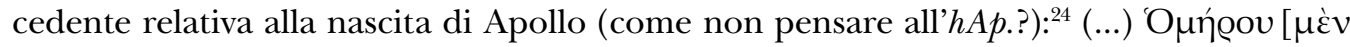

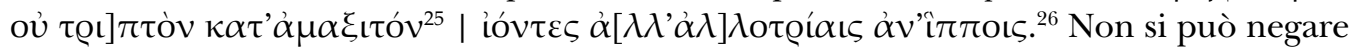
che diversi elementi suggeriscono la volontà di Pindaro di porsi come sistematizzatore

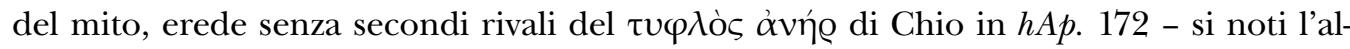
lusivo $\tau] v \varphi \lambda \alpha[\grave{i} \gamma] \dot{\alpha} \varrho \alpha \dot{\alpha} \delta \varrho \tilde{\omega} v$ $\varphi \varrho \varepsilon ́ v \varepsilon \varsigma$ al v. $17 .{ }^{27}$ In un altro frammento, $140 \mathrm{SM}$, restituitoci da P.Oxy. 403 e appartenente forse ad un peana, Pindaro introduce allo stesso

19 Serrao (1979: pp. 91-98).

20 Dubbi sull'esistenza di un'Artemis antimachea sono in Carrara (1986: pp. 213-216).

21 Si veda Faulkner (2015) per un'analisi interessante del "silenzio di Zeus" nello sviluppo della narrazione negli Inni omerici.

22 Per lo stesso proposito poetico, cfr. Sim. PMG 519 fr. 35.

23 Rutherford (2001: pp. 243-253). Si veda D’Alessio (1995) per un confronto con Parm., frr. 1+6 DK.

24 D'Alessio (1992: p. 174, n. 60) teorizza l'uso di éкós nel senso di una presa di distanza in materia di critica

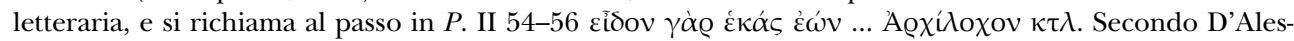

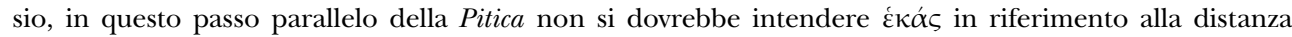
temporale, nel senso di "pur essendo lontano (nel tempo)": "una precisazione - egli osserva - a dir poco

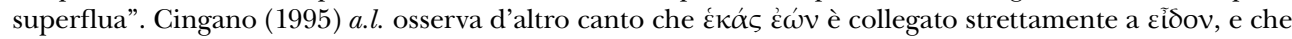
l'enfasi della formulazione annulli dunque la distanza cronologica tra i due poeti.

25 Per $\dot{\alpha} \mu \alpha \xi \iota \tau o ́ \varsigma$, si pensi a Parm. DK 28B 1.21, per cui Cerri (1999: pp. 170, 180).

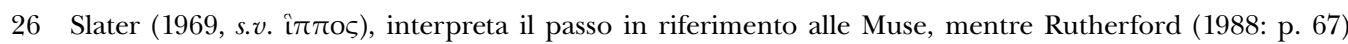
come appartenente ad un altro poeta, forse a Pindaro stesso. Cfr. a riguardo Lefkowitz (1991: pp. 155 e ss.) e Richardson (1985: p. 128). Altri luoghi in Pindaro di presa di posizione rispetto ad Omero: $N$. IV 69, P. XI 38, O. IX 80. Cfr. Call. Ep. XXVII 1 rispetto allo Høı́óov ó tøótos. Altri passi in cui Omero

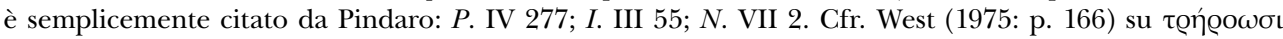

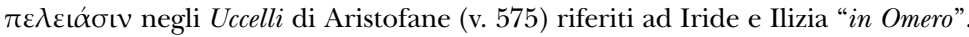

27 L'inno ad Apollo di Alceo (che conosciamo per via indiretta grazie ad Him. Or. 14.10 ss.) non doveva costituire, così come quello di Oleno, un modello antagonista a quello pindarico. Cfr. inoltre Sourvinou-Inwood (1979: pp. 231 e ss.) sulla costruzione dei quattro templi a Delfi in Pae. VIII come estensione dei vv. 274-279 nell'hAp. 
modo la narrazione dell'uccisione di Cicno su ordine di Apollo (b21-b33) opponendo il suo canto all'armonia di un anonimo rivale locrese (ő $\chi \mu \alpha \lambda \iota \gamma[\dot{v}$, v. 8), e assimilando

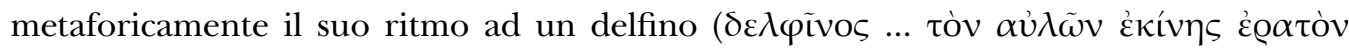
$\mu \varepsilon ́ \lambda o \varsigma, 15-17) .{ }^{28}$ Ciò detto, occorre ora seguire dall'interno l'andamento con cui Pindaro riplasma l'episodio della nascita a Delo. Tralasciando la parte programmatica del peana, si consideri ora quella finale dell'epodo, v. 42 e ss.

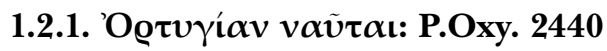

L'oggetto del canto diventano, nell'epodo di P.Oxy. 2440, Asteria e il suo trasformarsi in isola. ${ }^{29}$ Dai versi conclusivi sulle parole di Asteria in fuga da Zeus (42-44), Pindaro

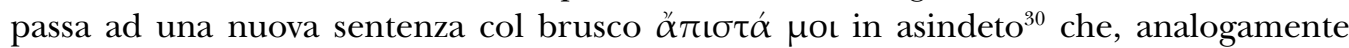

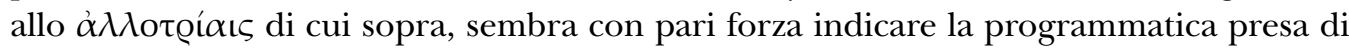
distanza da un'antecedente versione del mito. Arrivando Asteria alla cospicua roccia (v.

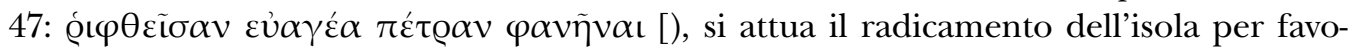

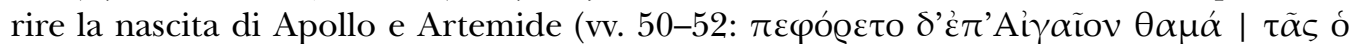

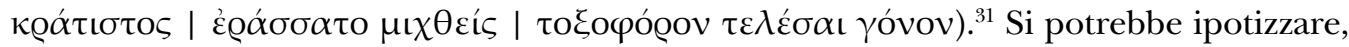
seguendo la coronide a chiusura dell'epodo, che Pindaro abbia voluto a questo punto chiudere il peana. Se così fosse, sarebbe legittimo chiedersi perché egli abbia voluto chiudere la triade senza completare la narrazione della nascita di Apollo, tema che motiverebbe per primo il distanziamento dagli ò $\pi \iota \sigma \tau \alpha$ citati. $^{32}$

Rispetto alle due soluzioni prospettate da Rutherford, ${ }^{33}$ ciò che mi sembra probabile è che la coronide stia qui ad indicare la fine di una sezione, ${ }^{34}$ incompleta e volta ad illustrare, dell'intero mito, i due momenti della fuga di Asteria e del suo radicamento di Delo consequenziali da un punto di vista narrativo. Due ulteriori indizi suggerirebbero che Pindaro stia qui progredendo nel proporre la propria versione del mito: il fatto che Delo non sia coperta d'oro e che il suo nome sia Ortigia, ${ }^{35}$ scelta che l'interpretazione tarda

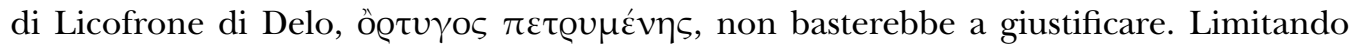
la nostra riflessione là dove la coronide chiude il movimento, si può per ora ipotizzare che Pindaro abbia innovato i fotogrammi dell' $h A p$. sostituendo Delo con Asteria, isola vagante che senza ricoprirsi d'oro si radica in mare.

28 Per lo scoliasta a $O$. X 17k e 18b si tratterebbe del cieco Xenocrito, capo della gilda spartana attivo nella

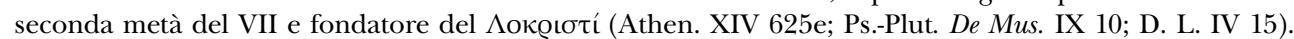

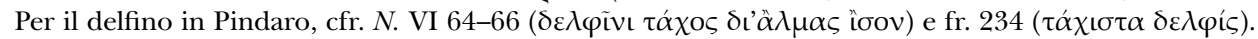

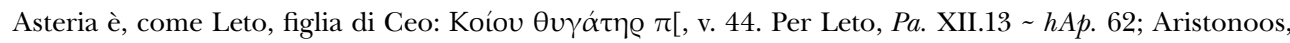
Pa. 5-6 in Powell (1925: p. 162); Call. hDel 150; A.R. II 710.

30 Cfr. Pind. O. I 52, N. IX 33. Sull'asindeto come indizio di lacuna, Führer (1967: pp. 72-75).

31 Cfr. Call. hDel. 53 e ss.; Aristot. fr. 48 R; Plin. NH 4.12.66. Sul $\tau \tilde{\alpha} \varsigma$ del v. 50, Schmidt (1975: p. 39).

32 Non vi sono indizi ulteriori che provino che la coronide voglia qui indicare la fine di tutto il componimento piuttosto che del solo epodo. La chiusura del passo mancherebbe in tal caso di un'opportuna formula clausolare, sempre presente negli altri peani: e.g. Pa. I 5, II 102, V 43, VI 178, XIV 35.

Rutherford (1988: p. 71).

34 Come anche in P.Lit.Lond. 46, P.Oxy. 659.

35 Càssola (1975: pp. 486-487); Sbardella (2012); Giuseppetti (2013: pp. 46-47). 


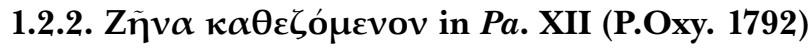

$P a$. XII a-e si struttura su particolari del mito assenti ma presupposti in $h A p$., primo fra tutti il ruolo di Zeus nei momenti precedenti e successivi alla nascita di Apollo. Menzionato nell' $h A p$. durante il primo accesso di Apollo sull'Olimpo (vv. 5-10), assente nel momento in cui Era tenta di osteggiare il parto di Leto, Zeus è in $P a$. XII colui che al contrario vigila dall'alto del Cinto lo svolgersi degli eventi (Z̃̃v $\alpha \kappa \alpha \theta \varepsilon \zeta$ ó $\mu \varepsilon v o v, 10)$. Tale cambiamento non può non ricordare Il. XI 182-183 ( VIII 51-52), in cui Zeus monitora dall'Ida lo sviluppo della battaglia. ${ }^{36}$ La nascita in simultanea di Apollo e Artemide è un ulteriore dettaglio da considerare, essendo la visione dei due come gemelli ( $\alpha \gamma \lambda \alpha$ òv $\dot{\varsigma} \varsigma$

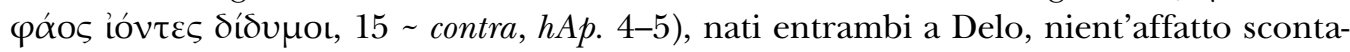
ta. ${ }^{37}$ Pindaro varia inoltre nella descrizione dell'atmosfera che circonda Leto partoriente: non più il corteo di Dione, Rea, Themis, Anfitrite, bensì le sole Ilizia e Lachesi assistono

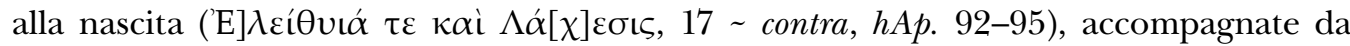

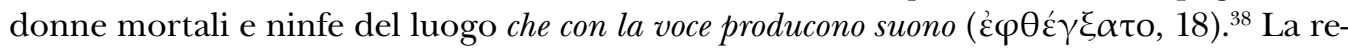
azione di Leto stessa cambia. Nell' $h A p$. il rifiuto delle isole e il ritardo di Ilizia, trattenuta da Era e condotta finalmente a Delo da Iris, provocano per nove giorni e nove notti il

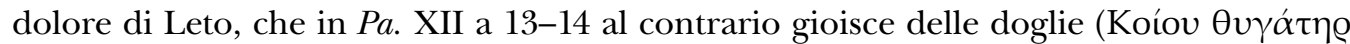

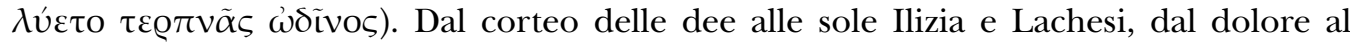
piacere di Leto generato dal parto di Apollo ed Artemide, dal silenzio di Zeus al suo patrocinare dall'alto: così Pindaro propone di affrancarsi dalle autorevoli versioni precedenti. Il $\pi[$ [Q]ovoí[ $\alpha$ del v. 11, restituito a fatica da Lobel 1961 benché privo di paralleli in Pindaro, ${ }^{39}$ ha poi portato alla sovrainterpretazione del ruolo di Zeus nella scena della nascita: che non vi sia forse al v. 11 - ci si è chiesto - un riferimento al patrocinio di Zeus e Atena Pronoia, concorrente quest'ultima alla Atena Pronaia di Delfi? ${ }^{40}$ Lasciando da parte, e irrimediabilmente aperta, la domanda, propongo ora di considerare l'invocazione a Delo dei frammenti 33c-d. In essa non si fa alcuna allusione alla supervisione di Zeus, e molti sono i richiami a $P a$. VIIb, come il $\tau \eta \lambda \varepsilon ́ \varphi \alpha v \tau o v$ in 33 c6 e il radicamento

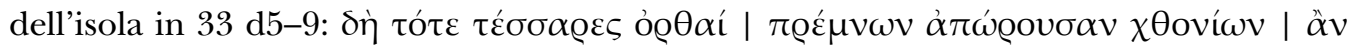

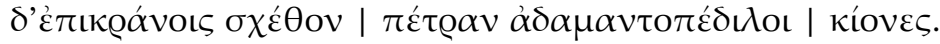

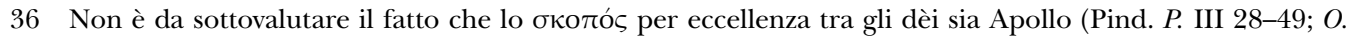

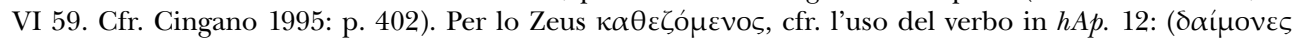

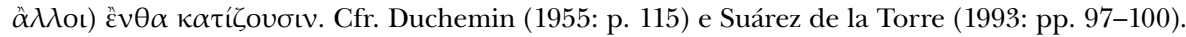

37 Si pensi a Pind. O. III 35; N. IX 4. Cfr. E. IT 1235 e ss.; schol. a E. Hec. 458. Sulla nascita di Artemide il giorno prima di quella d'Apollo, Apollod. 1.4.1; Serv. ad Virg. Ecl., 4.10. Cfr. Càssola (1975: pp. 581-582) per la versione nell'hArt. (XXVII). Cfr. anche $h H e r m$. 57-59 e hPan. (XIX) 27-47.

38 Cfr. $\pi \alpha \iota \alpha \nu \iota \kappa o ̀ v ~ \varepsilon ̇ \pi i ́ \varphi \theta \varepsilon \gamma \mu \alpha$ in Ath. 15.696. Per quanto riguarda Ilizia, altri autori, prima e dopo Pindaro, presentano quasi scontatamente la presenza di Ilizia al parto: Isyll. 52 e ss. (Powell 1925: p. 134); peana di Oleno in Paus. 8.21.3, 9.27.2; Call. $h$ Del. 257. Sulla statua di Ilizia a Delo, Hdt. 4.33.5 per cui Pingiatoglu (1981: pp. 33-36).

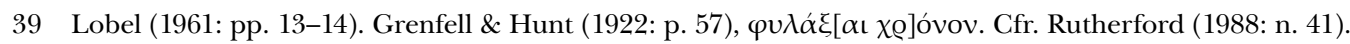

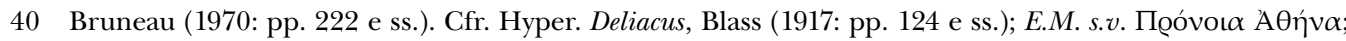
Macr. Sat. 1.17.55. 


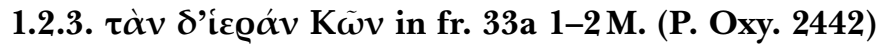

Parte secondo Snell di un prosodion cantato dalle Muse per Apollo all'interno di una nar-

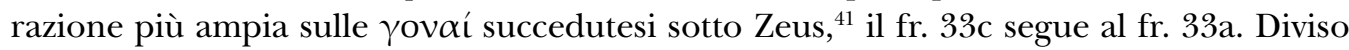
dal primo da un vacuum, vi si canta di Eracle a Cos. ${ }^{42}$ Supponendo una totale indipendenza del secondo frammento dal primo, si potrebbe immaginare che Pindaro vi abbia voluto narrare di Apollo dopo aver concluso un precedente intervento su Eracle. Come si vedrà anche più avanti, accenni dalla tradizione precedente e successiva ai frammenti suggeriscono tuttavia che la presenza ravvicinata di Apollo e Eracle non sia qui casuale. Oltre a ciò, i frr. 33a, 33c, 33d permettono di riconsiderare Cos, la sola località di quelle citate in $h A p$. 30-45 a non avere ricevuto la giusta attenzione, ${ }^{43}$ come luogo interessato da forme cultuali apollinee. ${ }^{44}$

La sequenza Cos-Delo ricorre a varie riprese, trattata diversamente secondo le esigenze del canto dai singoli autori. Nel catalogo delle isole toccate da Leto prima del parto, Cos è citata in $h A p$. 42 dopo Mileto e prima di Cnido, restia come tutte le altre ad accogliere la dea. Nell'Inno a Delo di Callimaco, Cos non è più l'isola che rifiuta, bensì quella che Leto stessa evita su profezia del figlio, che le parla dal ventre. Proprio lì un altro dio sarebbe infatti nato: Tolomeo Filadelfo. Callimaco non menziona Eracle, ma

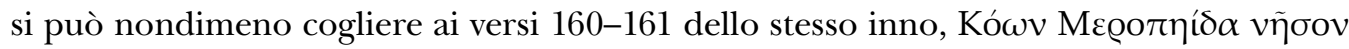

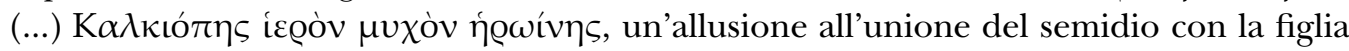
del re locale. ${ }^{45}$ Non si dimentichi inoltre che Elio Aristide (Or. 38 11-12 K, 147-148) paragona la metamorfosi di Cos dopo l'arrivo degli Asclepiadi a quella di Delo dopo la nascita di Apollo.

Quanto ad Eracle ed Apollo, un luogo parallelo significativo in Pindaro è il fr. 140a, ove Eracle attacca gli abitanti di Paro su ispirazione di Apollo: ${ }^{46} \xi \varepsilon v o \delta \alpha[i] \tau \alpha \beta \alpha \sigma \lambda \tilde{\eta} о \varsigma$

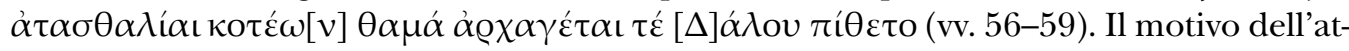
tacco fungerebbe verosimilmente da aition per l'istituzione del culto insulare di Apollo Dalios, invocato non a caso poco più avanti ai vv. 60-65. ${ }^{47}$ Come nel fr. 140a, anche in 33a Pindaro starebbe pertanto narrando la fondazione di un culto di Apollo inaugurato da un atto di giustizia di Eracle, all'interno di una strofe di impianto forse teogonico.

41 Per l'interpretazione dei frammenti come parti di un inno a Zeus, Furley \& Bremer (2001: vol. I, pp. 191-197; vol. II, pp. 133-139).

42 Per un caso simile, si veda infra 1.4.4.

43 Si pensi per l'appunto a Càssola (1975: pp. 490-491), che omette Cos.

44 Sappiamo ad esempio che nel 282 a.C. gli abitanti di Cos inviarono una theoria a Delo, prima attestazione di "a notably constant stream of Coan representatives within the following decades" per Sherwin-White (1978: pp. 91-92). Per un elenco delle theoriai inviate a Delo da Cos, Sherwin-White (1978: p. 91, n. 50). Cfr. Rutherford (2009). Per la cỉotó́ $\omega v \eta$, composta dal ramo d'olivo coperto di lana, cfr. Lycurg. fr. 82 Blass; Plut. Thes. 18 e 22; E. IT 1098-1110.

45 Cfr. Theocr. 17, in cui Cos prende tra le sue braccia Tolomeo Filadelfo appena nato (v. 58), pregandolo di onorarlo eternamente come Apollo ha fatto con Delo. Per la presenza di Eracle nella saga locale della Meropide, Bernabé (1987: pp. 133-135) per P. Köln inv. 5604 = MP3 96 (editio princeps Koenen \& Merkelbach 1976: pp. 3-26). Cfr. Sbardella (2000: pp. 33-34, 39-41) et Bernardini (2012).

46 Sherwin-White (1978: pp. 319-320).

47 Cfr. Bacch. Dithyr. 17.122-123. Calame (2009: p. 173). 
Lo stesso frammento testimonia parimenti la scelta di Pindaro di rendere Cos l'isola gemella di Delo, scelta che sarebbe ancora più emblematica se si accettasse di leggere con D'Alessio nei frr. 33a-d un inno ad Apollo piuttosto che a Zeus. ${ }^{48}$ Qualora si preferisse l'ipotesi snelliana di un un inno a Zeus, rimarrebbe comunque significativo il fatto che nel primo dei suoi inni (stando alla numerazione alessandrina) Pindaro abbia privilegiato l'aition di Cos rispetto alla nascita di Apollo a Delo. L'accostamente delle due isole, Cos e Delo, nello stesso papiro, ammissibile da un punto di vista tanto meccanico quanto logico, mostra così come Pindaro, recependo e sviluppando la citazione dell'isola dei Meropi nel primo catalogo geografico dell' $h A p$. (30-45), abbia operato una variazione allusiva rispetto alla geografia reale o fittizia del modello. ${ }^{49}$

\subsection{Ricostruire la Pythochtonia dell'hAp.: P.Oxy. 841}

Nei paragrafi che seguiranno si proporrà un confronto tra le sezioni eziologiche dell' $h A p$. e $P a$. VI, sì da chiarire alcuni particolari del culto delfico che ancora oggi coincidono con zone d'ombra dell' $h A p$. La scelta del Pa. VI, a noi trasmesso da P.Oxy 841, non è d'altronde casuale, essendo esso il più rappresentativo dei peani, oltre che uno dei meglio conservati. ${ }^{50}$

Vari aspetti legati alla trasmissione di $\mathrm{Pa}$. VI hanno attirato l'attenzione degli studiosi, tra cui l'invocazione d'Egina all'inizio della terza triade, poco familiare al tema della

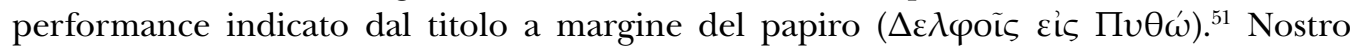
intento non sarà tuttavia chiarire il perché della presenza "intrusiva" degli Egineti o del riferimento all'eroe Aiakos. ${ }^{52} \mathrm{Al}$ contrario, si cercherà di capire come, partendo dalle tre immagini in $h A p .363 \sim 516-519 \sim 543-544$, Pindaro abbia elaborato un'eziologia alternativa della Pythochtonia sfruttando a tal fine il potenziale mimetico dell'epiphthegma posto solitamente a chiusura della triade. Di seguito, ci si concentrerà sulla seconda

48 D'Alessio (2009). Contra, Snell (19754: p. 87). Diversi sono infatti gli elementi a favore di Apollo nella titolatura dell'inno: un primo riferimento ad Ismeno e Melia (cfr. Schachter 1981: vol. I, pp. 15, 80-82); il canto delle Muse seguito dalla digressione teogonica su Apollo ed Eracle; il fatto che in diverse raccolte alessandrine l'inno ad apertura sia il più delle volte indirizzato ad Apollo, non a Zeus (con un'eccezione in Callimaco; si pensi all'inno ad Apollo d'Alceo, Heph. Poem. 3,6). Cfr. Pardini (1991).

49 Un altro caso in Pindaro di interpretazione del simbolismo geografico dell' $h A p$. è in P. Louvre E 7734 (+7733), in cui si narra di un "Apollo tra gli dèi come Echecrate tra gli uomini". La doppia celebrazione di Echecrate e Apollo, segnata da un coronamento nel corso di un banchetto sacrificale ad Orcomeno, si presta ad un'analisi incrociata con la cosiddetta sezione tebana, o itinerario beotico, dell'hAp. 225-245. Per la ricostruzione del testo e datazione su base paleografica di P. Louvre E 7734 (+7733), D'Alessio (2000). Per i problemi interpretativi di $h A p$. 229-238 in riferimento al rito del carro, si veda Teffeteller (2001). Per un'ipotetica tendenza anti-tebana dell'hAp., Burkert (1984: pp. 163-175) e Strauss Clay (1989: pp. 95-151).

50 Sul peana come forma poetica appartenente al genere della Kultlyrik, Herington (1985: pp. 3 e ss.); Käppel (1992: pp. 34 e ss.). Sui contatti con l'innografia egizia, Rutherford (2001: p. 11). Per l'etimologia di peana (in $\pi \alpha \imath \omega \dot{v}$ in hAp. $517 \sim$ PMG 922; cfr. ió $\beta \alpha \gamma \chi 0 \varsigma$ in Procl. Chrest. 320 b 31 e ss.), Gérard \& Rousseau (1968: pp. 164-165); Schwyzer \& Debrunner (1950: vol. II, p. 239); Rutherford (2001: p. 13, nn. 14, 16, 17); Calame (2009). Sui Theoxenia del sesto peana, si vedano Parker (2011: pp. 142-144), Jameson (1994: pp. 35-57 su SEG 50.168), Veyne (2000).

51 Rutherford (1997: pp. 1-21).

52 A riguardo, Radt (1958: pp. 163-168); Bona (1988: pp. 135-137); Cingano (1995: p. 193, n. 69). 
triade e, in particolare, sui vv. 121-122, che chiudono del peana l'episodio dell'uccisione di Neottolemo.

\subsubsection{Le tre immagini della Pythochtonia nell'hAp.}

Le prime due immagini della Pythochtonia nell'hAp. ritraggono la soppressione della dracèna, poi di Telfusa, e il corteo dei Cretesi al seguito di Apollo. Entrambe le immagini sono evidentemente legate alla fondazione del santuario a Delfi. La terza, che più ambiguamente preconizza il sopraggiungere dall'esterno di sconosciuti padroni (543-544), rinvia piuttosto all'amministrazione futura di Delfi da parte dei Cretesi, già sacerdoti. La successione dal $\pi v ́ \theta \varepsilon v$ del v. 363 (prima immagine) al $\pi \alpha \iota \eta o ́ v \varepsilon \varsigma / i \eta \pi \alpha ı \mid j o v ~ \alpha ̀ \varepsilon เ \delta o v$ dei vv. 516-519 (seconda immagine) non è tuttavia chiara: quale anello di congiunzione spiegherebbe, sul piano eziologico, il passaggio da Apollo Pytheios, poi Delphinios, ${ }^{53}$ all'Apollo corego del peana, donde la definizione dei Cretesi come $\pi \alpha \iota \eta o ́ v \varepsilon \varsigma ? 54$ L'iconografia pare non confortare l'ipotesi di derivazione della nuova epiclesi di Apollo Paion dal tradizionale î́ $\pi \alpha \tilde{\imath}$ ióv, gridato secondo la tradizione dagli astanti per incoraggiare lo slancio di Apollo contro la dracena. L'unico esemplare a noi pervenuto raffigura infatti il dio nell'atto di lanciarsi dalle braccia della madre in assenza del mostro. ${ }^{55}$ Attenendoci

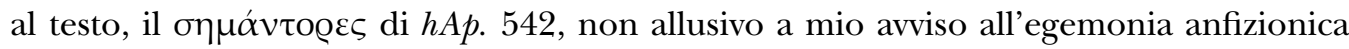
su Crisa, ${ }^{56}$ ci indica d'altro canto una possibile pista da seguire. Parola chiave del terzo

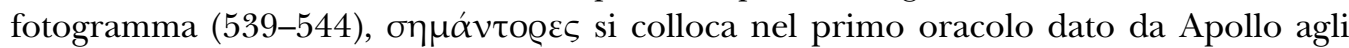
uomini, ${ }^{57}$ che evoca nell'immagine del buon sacrificio il coronamento della Pythochtonia e dell'installazione del culto apollineo a Delfi. Questo termine permette in parte di interpretare la sequenza uccisione-epifania-selezione dei sacerdoti-peana alla luce della versione di Pindaro in P.Oxy. 841.

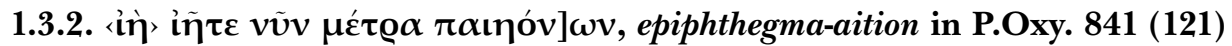

La narrazione di Pindaro in P.Oxy. 841 è costruita anch'essa su tre diversi momenti: l'uccisione di Achille da parte di Apollo (77-91), la presa di Troia da parte di Neottolemo per volere di Zeus (92-98), il giuramento di Apollo contro Neottolemo, reo di avere ucciso Priamo sull'altare di Zeus Herkeios (105-117). ${ }^{58}$ Da questi antefatti consegue

53 Herda (2008: pp. 14-18). Per Apollo Delphinios: Plut. Thes. 14, 1; LSCG 18; IG II² 4851.

54 Huxley (1975: pp. 119-124); Janko (1982: pp. 132, 200). Sul katakeleusmos di Sacada d'Argo (test. 10 Gent.Pr. = Athen. 13 p.610c), anticipatore delle forme del peana in età coeva all'hAp.: Str. 9.3.10; Ar. Ran. 207, Av. 1273; E. IT 1406. Cfr. Bowie (2010: pp. 52-53). Per Apollo Paion nel culto, IG I'3 383 e LSCG 18.

55 LIMC s.v. Apollon 993 = Roscher ML 3, 3407 fig. 4. Cfr. Fontenrose (1959: pp. 16-17, 550). Per l'etimolo-

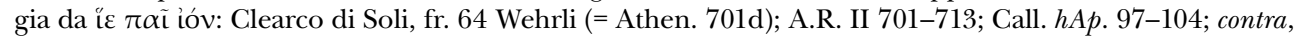
Ephor. FGrHist 70F31. Cfr. Williams (1978: p. 85).

56 Ipotesi di datazione in Guillon (1963: pp. 87, 95 e ss.), con bibliografia precedente; Froliková (1966: pp. 4-6); Janko (1986: pp. 119-121). Sulla datazione del 582, si vedano Allen \& Halliday \& Sikes (1936: p. 185);

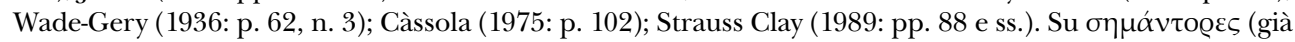
in Il. IV 431; VIII 127; XV 325; hDem. 131) nell'accezione di 'segnalatori', si veda Chantraine (1968) s. $v$.

57 Per gli oracoli d'Apollo, Fontenrose (1978: pp. 11-57); Parke \& Wormell (1956: vol. I, pp. 320-327). Per il legame tra l' $h A p$. 539-544 e la produzione delfica del terzo periodo, Colangelo (2016: pp. 10-12). 
l'uccisione da parte di Apollo di Neottolemo, arrivato a Delfi con smanie di conquista (117-120). La narrazione della morte dell'eroe è chiusa da un'appendice che, sigillando l'intero movimento della triade, si distingue formalmente dagli altri epiphthegmata, quasi a rappresentare essa stessa l'enunciato-aition da cui il lamento ritualizzato ì i ì $\Pi \alpha ı \alpha ́ \alpha$, ińıৎ sarebbe derivato. ${ }^{59}$

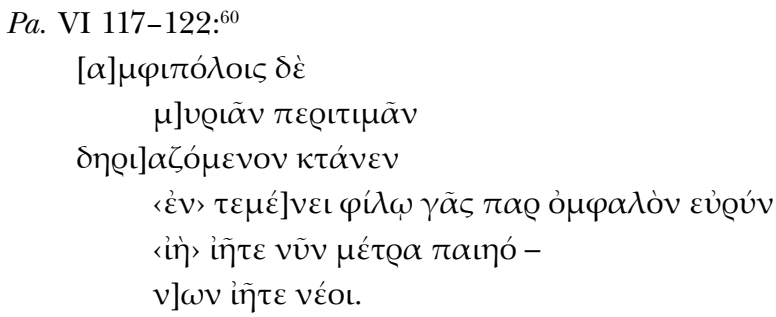

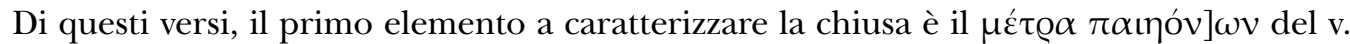

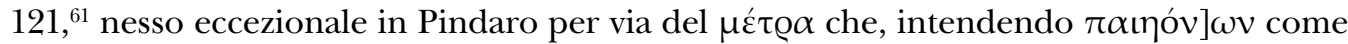
un genitivo oggettivo, si riferirebbe alla giusta misura del lamento trionfale dei véo prima che un nuovo movimento inizi. ${ }^{62}$ L'effetto transizionale sortito dal $\mu$ ćt@ $\left.\alpha \pi \alpha ı \eta o ́ v\right] \omega v$ farebbe dei versi 117-122 un epiphthegma a metà, ovvero un ritornello-lamento destinato non solo alla mera scansione del peana in stanze. Si potrebbe pensare che si tratti di un $\pi \varrho о к \eta ́ \varrho v \gamma \mu \alpha$, invocazione ai giovani spesso collocata ad inizio o a fine strofe per dare avvio all'elogio effettivo dei vincitori. ${ }^{63}$ Qui, tuttavia, oltre a non esserci vincitori da elogiare, l'io del poeta (o del corodidascalo) sembra piuttosto voler sollecitare i destinatari a perpetuare nel presente, $v \tilde{v} v$, ad ordine impartito, il momento evocativo, aition per l'appunto, del lamento. ${ }^{64}$ Oltre al $\mu \varepsilon ́ \tau \varrho \alpha$, anche lo ĩ $\tau \varepsilon$ soprende: grammaticalmente inspiegabile, per quanto l'ipotesi di interiezione al plurale sulla scia di $\tau \tilde{\eta} / \tau \tilde{\eta} \tau \varepsilon$ proposta da

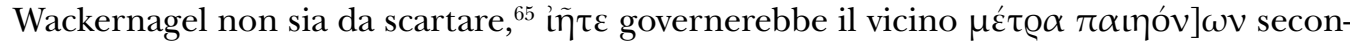
do un uso non frequente ma comunque attestato in altri componimenti cultuali. ${ }^{66}$ Tutti

59 Per la connessione tra peana e cretico, Blumenthal (1942: pp. 2361-2362).

60 Per la ricostruzione della preghiera finale di Pa. VI, D’Alessio \& Ferrari (1988).

61 Cf. Bacch. 16.8-9: $\pi \alpha ı \eta o ́ v \omega v ~ \alpha ̊ v \theta \varepsilon \alpha$.

62 Bundy (1962: p. 473). Per $\mu$ ćtœov in Pindaro, I. 1.61-64 (metron è la lunghezza che rende il componimento troppo breve per rievocare tutti i trionfi di Erodoto). Probabile che il modello qui sia Hes. Op. 694, con una sfumatura anche etica del termine.

63 E.g. Pind. I. 8.1; Bacch. 13.190.

64 Sul problema dell'identità della persona loquens nel melos, rimando alla ricca, ancora attuale, bibliografia in D'Alessio (1994).

65 Wackernagel (1943: p. 184). Cfr. Schroeder (1923: p. 538). Contra, Wilamowitz (1908: p. 348, n. 2) come forma paragrammaticale di ì $\mu \mathrm{t}$.

66 Pind. Pa. XII (P.Oxy. 2442, fr. 32 col. 2), vv. 3-4, 11-12, 19-20; Peana ad Asclepio in PMG 934. Per lo iñ $\tau \varepsilon$, il circonflesso è in P.Oxy. 841. Non c'è motivo di accettare lo î̀ $\tau \varepsilon$ di Schroeder, accolto da Turyn, Radt

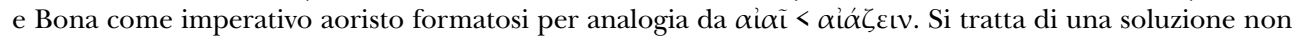
del tutto da escludere, ma troppo artificiosa e poco prevedibile a mio parere in un'ottica di efficacia performativa. 
questi elementi, che super-connotano l'epiphthegma di P.Oxy. 841 come enunciato-aition della stessa ingiunzione peanica, assumono ancora più importanza se si interpreta la

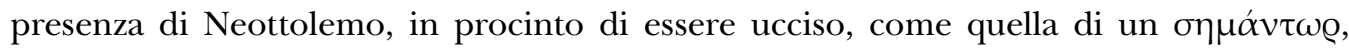

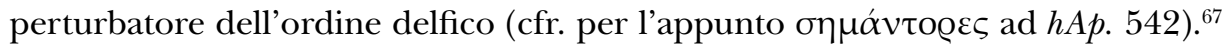

Le varianti del mito sull'arrivo di Neottolemo a Delfi confluiscono tutte verso una scena di sacrificio nei pressi dell'omphalos. Questo sacrificio si svolge a danno dell'usurpatore esterno (Neottolemo per l'appunto), che diventa a seguito della sua uccisione un eroe locale e themiskopos efebo. ${ }^{6}$ In suo onore, Pausania ci informa, si tenevano dei Theoxenia con enagismata. ${ }^{69}$ P.Oxy 841 narrerebbe dunque l'uccisione di un usurpatore esterno come momento coincidente con l'origine di una fondazione cultuale. La versione di Pin-

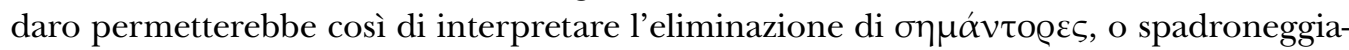
tori stranieri, come il motore principale di episodi di fondazione, oppure di riafferma-

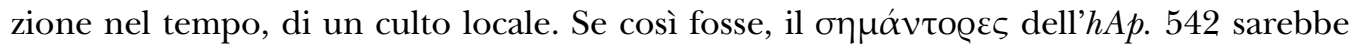
da leggere in senso meta-narrativo piuttosto che storico. Tramite questo raro vocabolo, Apollo alluderebbe nella sua profezia a sterminatori non autoctoni perturbatori dell'ordine delfico, la cui eliminazione avrebbe potuto apportare dei cambiamenti nella sfera

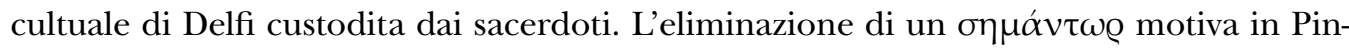

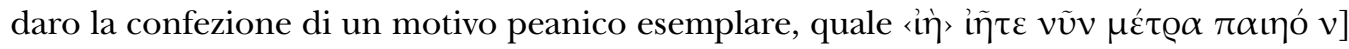
$\omega \nu$ iñ $\tau \varepsilon$ véot. In tal modo, proponendo una versione del mito incardinata sul motivo del perturbatore straniero, Neottolemo o seconda dracena da estirpare, ${ }^{70}$ Pindaro avrebbe recepito e modificato il coefficiente narrativo della sequenza "uccisione della dracena (poi soppressione di Telfusa)" - "manifestazione di potenza di Apollo" - "primo peana della Pythochtonia" nell' $h A p$.

\subsection{Ritorno all'epiteto di Apollo nell' $h A p$. Stesicoro, Pindaro, P.Lit.Goodspeed $2^{71}$}

I riusi dell'epiteto di Apollo nei papiri di epoca pretolemaica e tolemaica possono dividersi in due classi: nella prima rientrano i riusi dell'epiteto nelle trasposizioni letterarie

67 Si pensi anche al fr.140a, vv. 55-57, del P.Yale 18 (inv. 44), in cui Eracle uccide i Parii su ordine di Apollo,

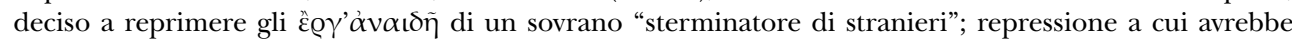
fatto seguito la fondazione del culto di Apollo Dalios.

68 Apollod. Epit. 6.14; E. Or. 1655-1657; Str. IX 3.9; schol. vet. a Pind. N. 7. 40 (5b); E. Andr. 1147-1149, 1161-1165; Paus. I.13.9. Cfr. Suárez de la Torre (2007: pp. 154-155). Per l'uccisione nei pressi dell'omphalos, Detienne \& Vernant (1979: pp. 215-237). Quanto al themiskopos efebo, Pavese (1993: pp. 469-479). Sulla Ephebenfest di Delfi, Burkert (1975: pp. 1-21). Su Apollo Lykeios protettore dell'efebia, Graf (1985: pp. 220-226). Si ha conoscenza del culto delfico ad Apollo Theorios (Plut. E Delph. 394a; Pind. N. III 70 con sch. a.l. = III, p. 59 Drachmann). Sull'eroizzazione dell'eroe cantato in Pindaro, Currie (2005: pp. 120-157).

69 Paus. X 24.6. Cf. Pind. N. VII 34-38; schol. Pind. N. VII 62a, 68a; Pherekydes FGrH 3 F 64 J. (schol E. Or. 1654f); E. Andr. 1264. Cfr. Fontenrose (1960). Sugli enagismata, Parker (1996: p. 39). Cfr. Paus. IX 10, 4, e Procl., Chrest. ap. Phot. Bibl. 239 (p. 321 a-b Bekker) per il rito analogo della daphnephoria.

70 Altri luoghi sembrano confermare l'assimilazione, debitrici tutte da Pindaro, tra cui i due peani delfici: Powell (1925: pp. 141, 149). Si veda e.g. Lycophr. Alex. 327 e Virg. Aen. II 471-515.

71 Si considererà nelle pagine successive il solo caso dell'epiteto apollineo, lasciando da parte quello altrettanto delicato dell'epiclesi. Ci si limita qui a rinviare, per un nuovo approccio sull'epiclesi come lente d'ingrandimento sul politeismo antico, a Brulé (2005: p. 333). Contra, Parker (2005: p. 338). Cfr. anche Wallensten (2008). 
delle vicende del dio; nella seconda, le epiclesi ad Apollo in preghiere e invocazioni differenti, nei moduli enunciativi, dal modello proemiale. La classe a costituire l'oggetto della nostra riflessione sarà la prima, essendo la seconda, legata alle diverse percezioni di Apollo in epoca post-classica, difficile da trattare nello spazio e nei limiti di una ricerca dedicata in primo luogo a problemi di intertestualità. ${ }^{72}$ Lo Stesicoro di Lille sarà il

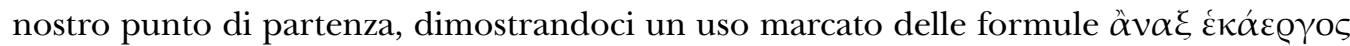

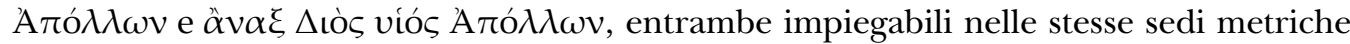
senza essere per questo intercambiabili in termini di significato. ${ }^{73}$

\subsubsection{La distribuzione dell'epiteto di Apollo nell'hAp.}

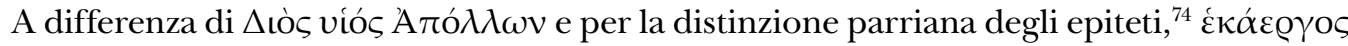
- attributo che qualifica uno dei possibili modi di agire della divinità, derivante per al-

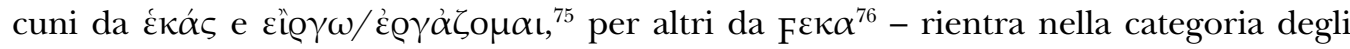
epiteti portatori di senso da un punto di vista sia formale (metrico) sia concettuale

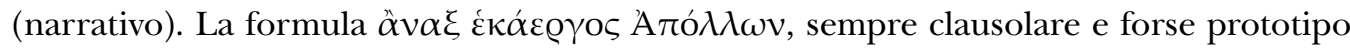

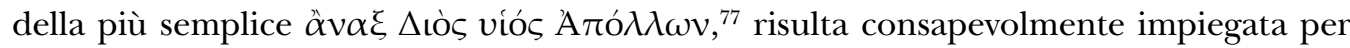

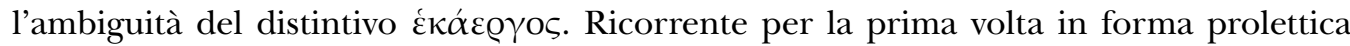

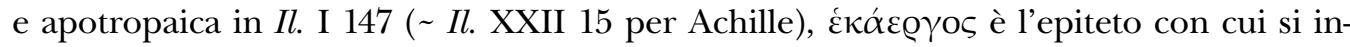
voca Apollo nel peana degli Achei (474), che ricambia, Fek $\alpha$, l'offerta del canto acheo

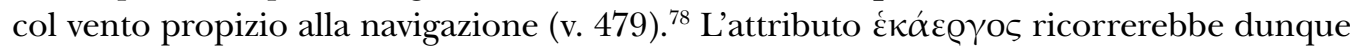

72 Per una stratigrafia del cambiamento religioso di Apollo riflesso in arte, si veda Micheli (1995: p. 5). Cfr. Monbrun (2007: p. 185). Per la henosis tra le figure di Apollo, Dioniso e Pan, rimando a Giordano (2011), con i riferimenti a Massa (2006-2007) e Maas \& MacIntosh Snyder (1989: p. 69). Ci si limiterà qui a menzionare, tra i papiri della seconda classe, $P M G$ III, trasmesso da P. Louvre 2396, e PMG VI (Par. Bibl. Nat. Suppl. gr. no. 574). Il secondo riporta un inno ad Apollo-Helios e Selene per l'ottenimento di una rivelazione in sogno: $P M G$ VI 6-7. In questo stesso inno, il contatto col dio è ricercato attraverso un'invocazione in forma espansa in cui l'epiclesi, in un contesto di pratica di magia simpatetica (oneiromanzia), è piegata retoricamente all'ottenimento del favore del dio. Si pensi all'analogo caso di $P M G$ IV 2714, inno magico a Selene, Ecate e Artemide, per cui si rinvia a Faraone (1997). Sull'invocazione espansa, quasi erratica, di Apollo, si pensi a PMG I 310 e Oracl. Sibyl. III 1. Per Apollo come il più invocato nei papiri come divinità mantica, Suárez de la Torre (2009: p. 21).

73 Si veda a riguardo Cantilena (1982: p. 205). Per l'uso esteso della formula omerica nello Stesicoro di Lille come indizio di dipendenza di Stesicoro da Omero, per primo Gallavotti (1980-1981: pp. 26-27, 413-433). Cf. Lazzeri (1999) e Gentili (1977: n. 52).

74 Parry (1928: pp. 153-165), per la struttura nome-epiteto più in generale si vedano pp. 119-124. Per una visione organica degli studi parriani, Di Donato (1969). Sul thematic heritage dell'epiteto ornamentale, cfr. Nagy (1981: pp. 2-3), e Hainsworth (1978: pp. 45-50) sui vari stadi di maturità della formula contenente un epiteto del secondo specimen.

$75 \quad E M$ 319. 51-52; Eust. 72. 15. 138. 23.

76 Chantraine (1968: vol. I, pp. 327-328); Boisacq (1916: pp. 232-237); Schwyzer (1939: vol. I, pp. 439-440, n. 8).

77 Parry (1928: pp. 177-178); Hainsworth (1968: pp. 5-8, 31).

78 Cfr. Il. V 34-35, o Il. IX 559-560 con l'episodio di Marpessa. Ancora più illuminante è $I l$. V 439, in cui

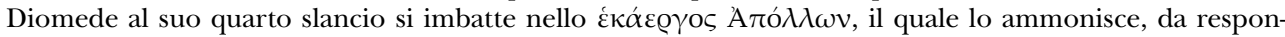
sabile sia della vittoria che della possibile disfatta dell'eroe, sui limiti dei mortali; Diomede obbedisce ed

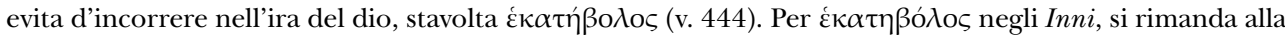
breve trattazione in Tsitsibakou-Vasalos (1985: pp. 126-128). Cfr. Nagy (1979: pp. 143-144, 293-294) su 
per definire il ruolo contraddittorio di Apollo, preservatore e distruttore insieme (con

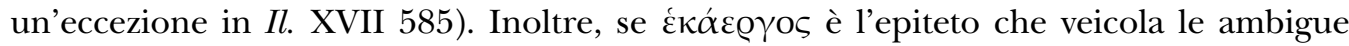

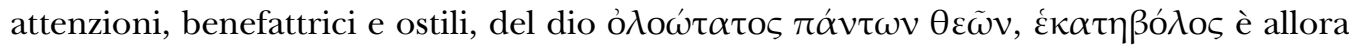
il complementare usato per il materializzarsi del suo potenziale nemico.

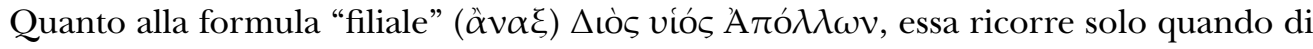
Apollo si vuole dare l'immagine di supremo esecutore della volontà paterna: così è, ad esempio, in $I l$. XVI 719, quando Apollo, facendo mostra dei suoi doveri filiali, incoraggia Ettore a combattere. Di questa stessa autorità Apollo è investito in Il. XVII 326, quando incita al combattimento Enea per evitare che Troia cada v́tę̀ $\Delta$ iò $\alpha$ Ĩo $\alpha v$ nelle mani

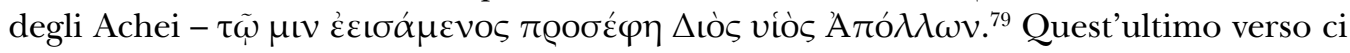
mette di fronte ad una costante da tenere a mio avviso in considerazione, ovvero l'appa-

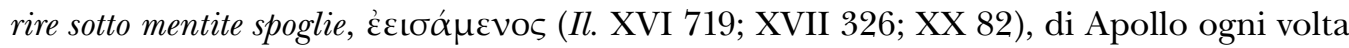
che il suo intervento si qualifica in funzione del suo essere figlio di Zeus.

Considerando ora il nostro $h A p$., la logica distributiva degli epiteti è alquanto oscura. Altrettanto difficile è capire quale associazione il rapsodo volesse evocare nel definire Apollo in un modo piuttosto che in un altro. L'impiego degli epiteti e delle formule appena viste è discontinuo a seconda delle sezioni, e:

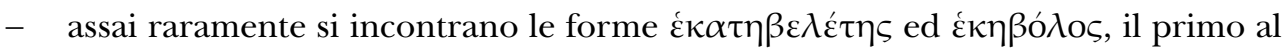
v. 157, il secondo ai versi 45 e 177;

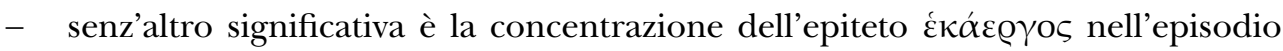

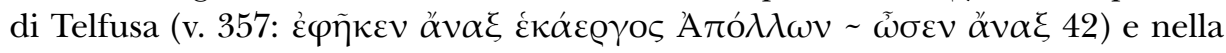

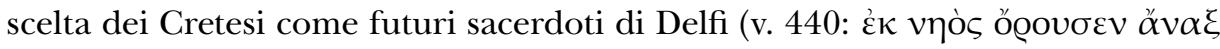

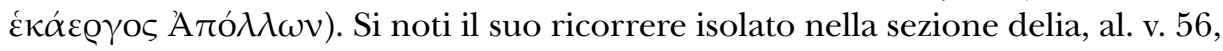
proprio quando Delo si rivolge ad Apollo già cresciuto e pronto a fondare un

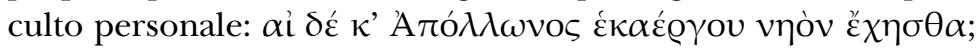

- la qualifica di Apollo come $\Delta$ iòs viós è significativamente assente nella cosiddetta sezione delia, costantemente evocata invece, con i verbi del proferire e del co-

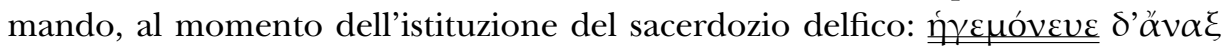

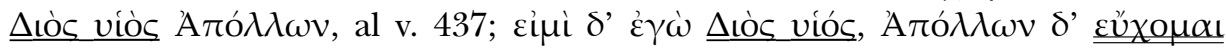

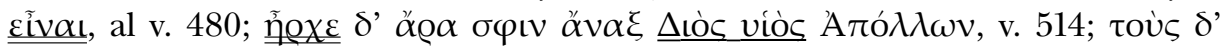

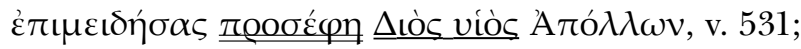

- distintivo della parte centrale dell'hAp., dal dialogo tra l'aedo e le Deliadi sino

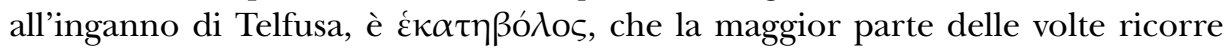
ad apertura di frase nel catalogo relativo al viaggio continentale di Apollo. Qui l'epiteto si combina a verbi indicanti la ricerca, il superamento di un confine e il

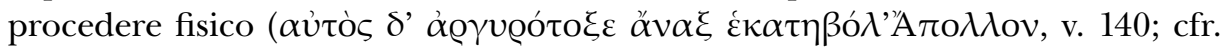
vv. 215, 222, 229, 239).

Ciò detto, sembra che la definizione per epiteti di Apollo si costruisca nell' $h A p$. su tre diversi livelli: l'essere figlio di Zeus, l'intervenire da lontano, il materializzare la sua forza d'azione a distanza. Questi tre livelli sono a loro volta in grado di circoscrivere tre diver-

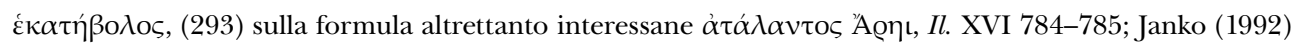
ad Il. XVI 702-706. 
se sfere di narrazione dell' $h A p$., che riflettono i tre modi d'azione del dio, il vero oggetto

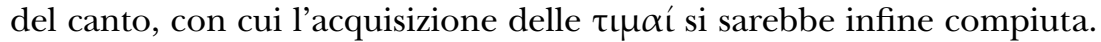

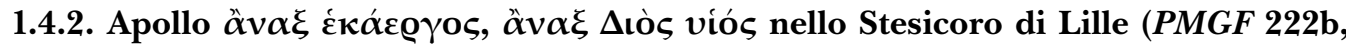 204-210) ${ }^{80}$}

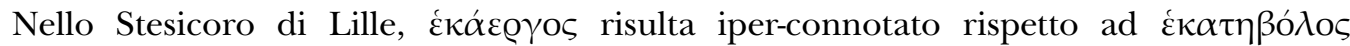

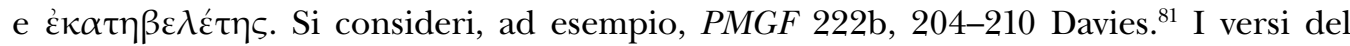
passo appartengono ad una sezione che segue all'intervento della regina madre, la quale chiede ad un "tu" (certamente al Tiresia del v. 234) di non aggiungere $\chi \alpha \lambda \varepsilon \pi \alpha i$ $\mu \varepsilon \varrho i ́ \mu v \alpha \iota$ agli $\ddot{\alpha} \lambda \gamma \varepsilon \alpha$ del passato, così come di non predire $\varepsilon \dot{\lambda} \lambda \tau^{\prime} \delta \varepsilon \varsigma \beta \alpha \varrho \varepsilon \tilde{L} \alpha$ เ. La richiesta così si chiude, con il lessico tipico della cosmologia empedoclea: ${ }^{82}$

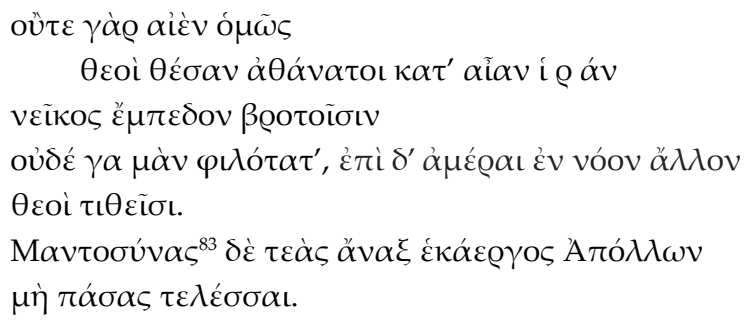

Nel quadro di instabilità cosmica, segnata in questi versi dal sottostare di philia e neikos al volere divino, la madre dello Stesicoro di Lille interviene rivolgendosi direttamen-

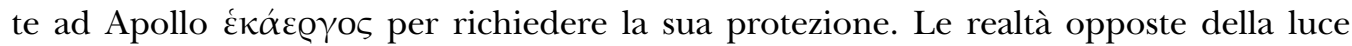
(204-208) e dell'ombra (211-217) dell'oikos coincidono con le situazioni critiche in cui Apollo dall'arco che canta acuto interverrebbe, benefattore trionfante come distruttore. ${ }^{84}$ L'illusorietà della condizione umana è un dato che risulta enfatizzato dal $\theta \varepsilon o i ̀ ~ \theta \varepsilon ́ \sigma \alpha v$ del v. 205, formula che indica in Omero una situazione di chiara avversità divina per i mortali. ${ }^{85}$ I vv. 204-217 del frammento mostrano dunque un'interessante stratificazione dei poteri divini per il comando su philotes e neikos, alla quale corrisponde l'impiego della

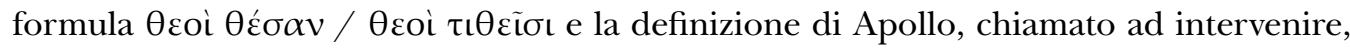

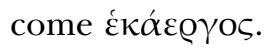

80 A ragione si può vedere nello Stesicoro di Lille uno dei possibili antecedenti dell' $h A p$., insieme all'inno ad Apollo di Alceo. Per maggiori dettagli sul rapporto tra Alceo e l'hAp., si vedano Page (1955: pp. 249-250) e Suárez de la Torre (2002: pp. 155-178).

81 Si consultino anche l'editio princeps di Ancher \& Boyaval \& Meillier (1976), con gli additamenta di Ancher (1978); Bollack \& Judet de la Combe \& Wismann (1977); Parsons (1977); Gentili (1977) e Hutchinson (2001).

82 Cfr. Solmsen (1980) per i richiami cosmici nell'inno empedocleo ad Apollo (B 134 DK), su cui si veda infra, 15. Cfr. Farrell (2014: pp. 2-3) per una nuova visione della fortuna dell'Apollo di Empedocle.

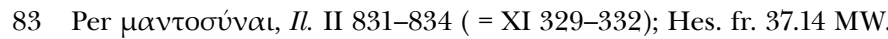

84 Giordano (2010: pp. 133-134), sul semantema dell'arco di Apollo che canta acuto in Il. I 46-149.

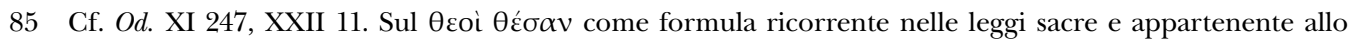

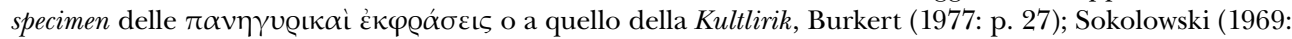
pp. 21-22, 26). 
La localizzazione dell'epiteto d'Apollo in PMGF 222b induce a riflettere sulla concentrazione degli attributi del dio nei tre movimenti dell'hAp. Sorprende, innanzitutto, che Apollo non si qualifichi come figlio di Zeus proprio nella sezione dedicata alla sua

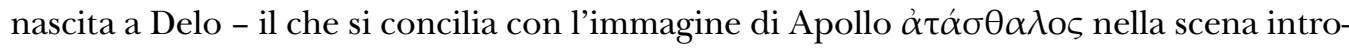
duttiva, $h A p$. 1-5. La fondazione del sacerdozio a Delfi è, al contrario, il momento in cui il legame filiale con Zeus diventa elemento identificativo, come se nella gestione del proprio culto Apollo si confermi il supremo esecutore dell' $\alpha$ ĩ $\sigma \alpha$ paterna. Il primo epiteto con cui si qualifica Apollo dopo la sua nascita è inoltre $\varepsilon$ k $\alpha \tau \eta \beta o ́ \lambda o s$, il complementare,

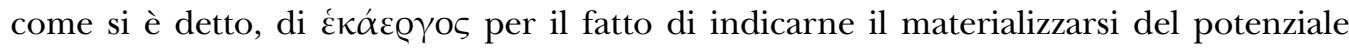
nemico. Lo stesso $\varepsilon \kappa \alpha \tau \eta \beta o ́ \lambda o \varsigma$ ricorre regolarmente ogni volta che il procedere fisico del dio, prima di una sua manifestazione di potenza, è al centro della narrazione. $\dot{\alpha} \tau \alpha-$ $\sigma \theta \alpha \lambda i ́ \alpha$, vendicatività, terribilità: questo è il tratto messo in maggiore evidenza quando si narra di uno spostamento fisico del dio; vendicatività che si spegne nel momento in cui Apollo entra in dialogo con Telfusa. Nell'episodio che vede il dio reprimere la sua

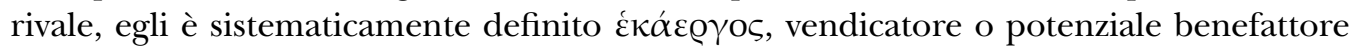
nei confronti di un interlocutore ingannevole.

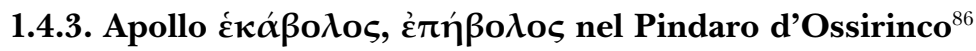

Se per una parte della critica l'Apollo degli epinici di Pindaro è il musico protettore della divinazione - l'Apollo, insomma, degli ultimi versi dell'hAp. -, per un'altra egli sarebbe il vendicatore per eccellenza che, confondendosi nella turba dei combattenti, ne esce vittorioso - l'Apollo, dunque, che sopprime Telfusa. Nei Peani l'immagine dell'arciere vendicatore è dunque preponderante, ricorrendo a quattro riprese $\varepsilon \kappa \alpha \beta o ́ \lambda o \varsigma ~(P a$. VI 80, 11;

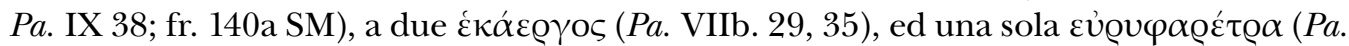

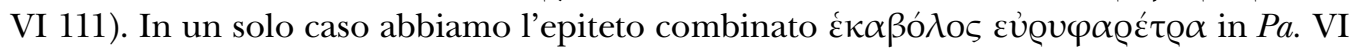
3 , dato dall'unione di due attributi indicanti la violenza del dio, adatti a rievocare l'uc-

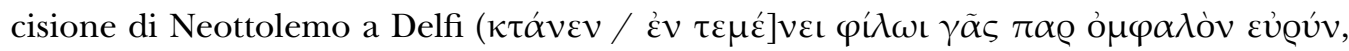
119-120). ${ }^{87}$ Abbiamo, al contrario, solo due esempi di epiteti costruiti sul determinante

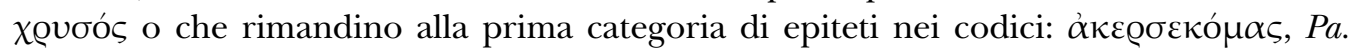

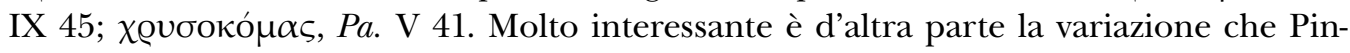

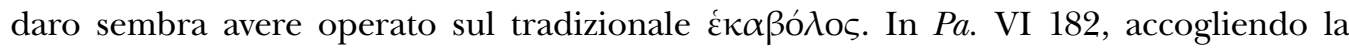
congettura di Ferrari $\dot{\varepsilon} \pi \alpha \beta o \lambda[\dot{\varepsilon}]$ ov $\tau(\alpha),{ }^{88}$ si avrebbe coniata la definizione per Apollo di

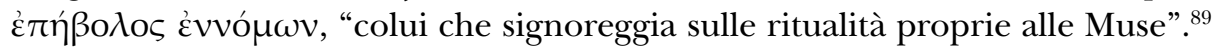

86 Un primo esempio di studio sull'epiteto d'Apollo in Pindaro è in Stefos (1975: pp. 221-251); cfr. Kyriazopoulos (1988: pp. 365-368). Per l'Apollo musico, si veda Duchemin (1955: pp. 106, 115). Per l'Apollo vendicatore, Ruck (1976: pp. 235-252); Méautis (1962); Defradas (1972²: pp. 149 e ss.).

87

Cfr. Il. XXIII 359; E. Andr. 1149-1152; Str. IX 421.

88 P.Oxy. 841 (fr. 15, col. 1), metà del II d.C., in Grenfell \& Hunt, V, 1908 + PSI 147 (frr. III, XI, XII, V) in Vitelli (1913: pp. 73-79). Sui calcoli delle lacune dei due papiri, Radt (1958: pp. 32-33); D'Alessio - Ferrari (1988: p. 176).

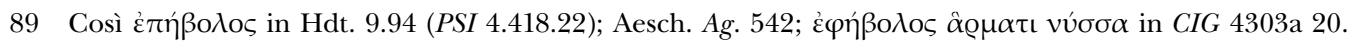
Cfr. D'Alessio - Ferrari (1988: p. 177) sui vv. 182-183. La funzione coregica d'Apollo è attribuita alle

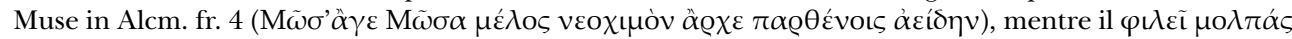

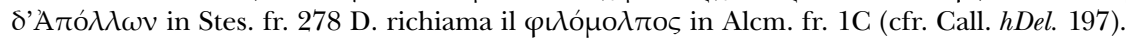




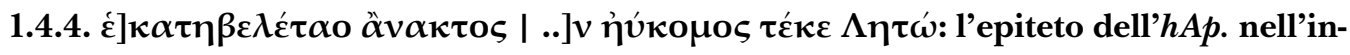 no lagide in P.Lit.Goodspeed 2}

Soffermandoci su una categoria specifica dei papiri della prima classe, contenenti cioè inni o antologie di inni, scopriremmo che Apollo come destinatario della preghiera vi è pressoché assente. Al contrario, si incontrano Afrodite (e.g. P.Köln VI 242; ${ }^{0}$ P.Berol. inv. 21160, in cui compare insieme ad Anubi), Cibele (e.g. PSI I, Bartoletto 1965: pp. 9-15), Hellos (e.g. O. Edfu III 326), Poseidone (e.g. P.Köln I.6, che doveva in realtà contenere un inno a tutti gli dèi), Ermes e Antinoo (P.Oxy. VI 3537). Nella raccolta di Powell, la presenza di Apollo sembra essere ugualemente evanescente, ricordata a margine del peana di Dioniso (Phil. Sc. 62). ${ }^{91}$ A ragione, pertanto, P.Lit.Goodspeed 2 può dirsi un unicum nei repertori antologici di inni (o inni-encomio?) su papiro. ${ }^{92}$ Oltre ad essere uno dei pochi esempi di raccolte innografiche, esso è infatti l'unico a restituire un inno dedicato con molta probabilità ad Apollo. ${ }^{93}$

Dei versi trascritti nel verso di questo rotolo vergato tra il II e il III d.C., ciò che a noi interessa è il carme teogonico che occupa la sesta e la settima delle dodici colonne. In esso si tramanda un inno ad Apollo e Zeus, ${ }^{94}$ composto molto probabilmente in epoca ellenistica o (pre)tolemaica a giudicare dalla tecnica di versificazione. ${ }^{95}$ Parte di un inno ad Apollo i cui vv. 1-5 sarebbero riferiti all'Egitto e i 9-13 alla ritirata dei Galati dall'invasione del santuario delfico - lettura che non trova conferma nel testo ma che continua ad essere oggi discussa ${ }^{96}$ - il testo di questa colonna ha susciato l'interesse dei filologi per le sue peculiarità ortografiche. ${ }^{97}$ La sesta colonna del papiro presenta altri elementi che meriterebbero pari attenzione, primo fra tutti il ritardo con cui il nome di Apollo compare rispetto alle gesta di Eracle. L'azione dell'eroe doveva, a quanto pare, essere più importante per la definizione di una cornice di riferimento per l'uditorio.

90 P.Köln VI 242, inv. 20270-4. LDAB 6860. Cfr. Maresch (1991: pp. 26-51).

91 Il nome d'Apollo compare in Powell (1925) in Sim. 2; Is. 19; Euph. 95, 102; Phoen. 2.2; Arist. 1.5, 2.7; Alex. Aet. 13; Pac. Sel. I, Phil. Sc., 62, fr. 4; Hymn. Dact. Id. 10.

92 GDRK I2; LIX 179-199; Preisendanz PMG 1-2 (Pack 1065, 1863), 7 (Pack 1965, 1967). Si ricordi anche il papiro di Parigi (Paris. Bibl. Nat., Suppl. gr. no. 574). Quanto all'inno-encomio per fondazioni o celebrazioni di città, si veda Hunter (2003: pp. 8-24).

93 Non si è considerato rilevante ai fini della nostra indagine P.Lond. III 970, che trasmette delle informazioni a mio parere non sufficientemente coerenti sulla figura di Apollo. Testimone di un componimento che a stento definirei inno consultandone l'edizione Kenyon (1907), in esso mi pare di leggere il nome di Apollo una sola volta insieme a quello di Galatea, ragione per cui si è preferito interpretarlo come un epyllion all'interno di un inno perduto. Cfr. Körte (1932: p. 30): forse un epitalamio?

94 Nelle colonne I-V, forse anche nell'XI, è conservato un inno a Afrodite, con riferimento ad Arsinoe

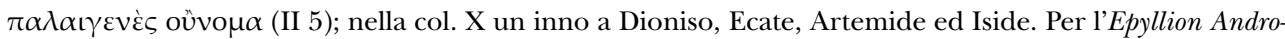
medae (col. VIII-IX), si veda Powell (1918: p. 127).

95 Meliadò (2004). Cfr. Powell (1918: pp. 82-89); Cameron (1995: p. 271, n. 47). Per la storia controversa del ritrovamento, Barbantani (2008: pp. 1-2).

96 Meliadò (2004: p. 111); cfr. Powell (1918: p. 126).

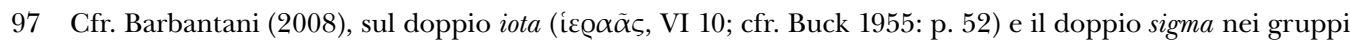

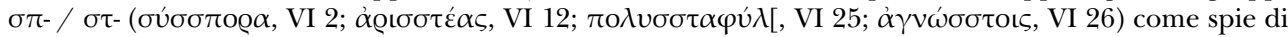
una revisione finalizzata all'esecuzione pubblica del testo. L'inserzione di consonante prima di un attacco con aspirata, $\kappa \chi\left(\dot{\varepsilon} \pi i{ }^{\prime} \kappa \chi \emptyset o v i ́\right.$, VI 16) viene spiegata come tentativo, a fini soprattutto prosodici e performativi, di creare lo stacco nella sillabazione e renderlo più marcato all'orecchio di chi ascoltava. 
Prima di incontrare Apollo, si dovrà dunque passare per i versi 9-14, in cui si menziona

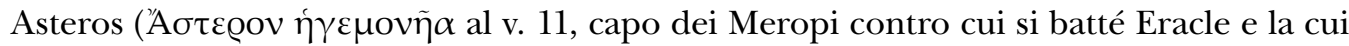

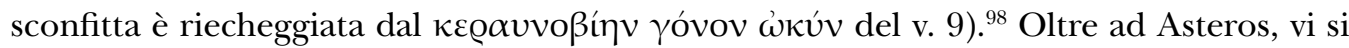
ricorda la vittoria di Eracle contro i Giganti e i Meropi - se si accetta l'integrazione al v.

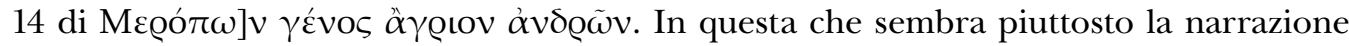
di una spedizione civilizzatrice di Eracle, da cui la definizione di "carme cosmogonico"

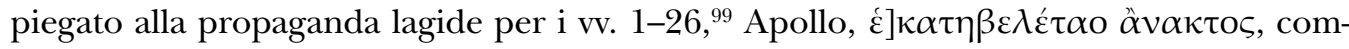
pare con relativo ritardo al v. 17. Due versi più avanti se ne evoca il luogo di nascita (19:

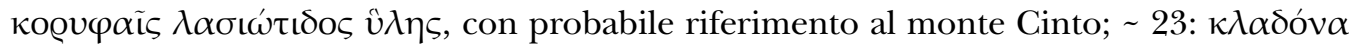

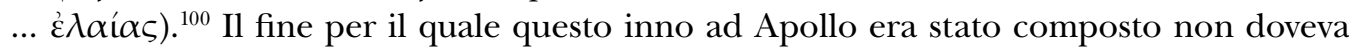
essere diverso da quello dell'inno ad Afrodite-Arsinoe. Questo, composto per l'anti-

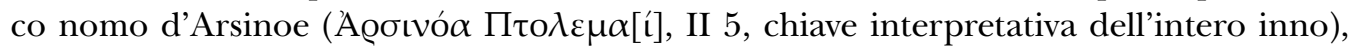
fu concepito come chiaro strumento di propaganda per assicurare e pubblicizzare la continuità dinastica entro l'orbe tolemaico. Allo stesso modo, l'episodio di Eracle rimanderebbe alla sfera civilizzatrice e di ordinamento cosmogonico. ${ }^{101}$ Questo inno sembra pertanto innestarsi nella produzione lagide attraverso un sottile gioco di richiami espressivi tra la sesta colonna del papiro e l'hAp. - e, più in generale, gli Inni omerici: il

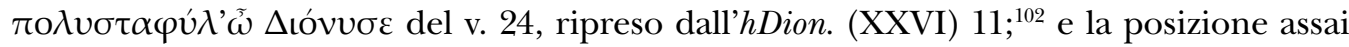

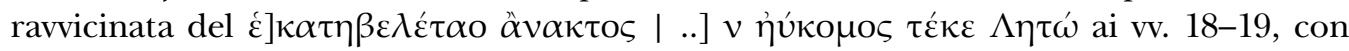
la stilizzazione dell'epiteto di Apollo che troviamo con la stessa vicinanza e nella stessa

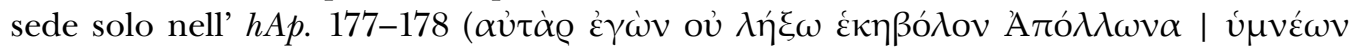

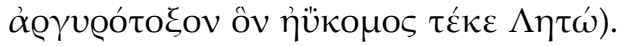

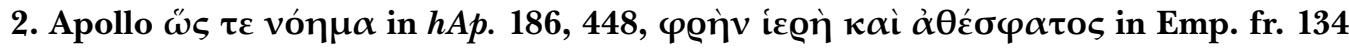 DK ${ }^{103}$}

La natura degli dèi nelle dottrine presocratiche offre ancora oggi materia di dibattito: si pensi allo Zeus del fr. 6 di Empedocle, ora fuoco ora etere nelle esegesi moderne da Knatz a Kingsley, ${ }^{104}$ ma anche al fr. 134, che ci pone di fronte ad un tipo di invocazione

98 Così è anche nella Meropis, di cui P.Köln III 126, attribuibile al Peri Theôn di Apollodoro (ora SH 903 A). Si veda Führer (1977: p. 42); Lloyd-Jones (1984); Breglia Pulci Doria (1996: pp. 385-398). Testi chiave per la vittoria di Eracle sono Od. XI 617-626; Hes. frr. 25. 24-33, 33. 24 MW; E. Herc. fur. 1250; Call. Aet. fr. 23 19-20 Pf.; Nonn. D. 25. 197. Per quanto riguarda i Meropi, non è da escludere la sovrapposizione con i Giganti (cf. Philostr. Her. 8.14), la cui sconfitta ad opera di Eracle è parafrasata in Quint. Smyrn. 8.6.71; Str. 7, fr. 58 .

99 Cf. Virgilio (1993: pp. 43-49). Si confronti il P.Oxy 2442 fr. 1 per una profezia di Apollo elogiativa della politica del Filadelfo, $\sigma \omega \tau \tilde{\eta} \mathrm{\varrho}[\alpha]$ in Milet. I 3. 139 (cca. 262 a.C.). Per l'inno ad Afrodite-Arsinoe, si veda Barbantani (2005b: pp. 143-147). Per l'aspetto nuziale dell'inno, Weber (1993: pp. 260-261).

100 Cf. Lycurg. fr. 82 Blass; Plut. Thes. 18, 22; E. IT 1098-1100. Cfr. Bernardini (2009); Hardie (2000).

101 Sul carattere civilizzatore e fondatore delle imprese di Eracle, cfr. Brelich (1958: pp. 193-196), e Jourdain-Annequin (1988-1989).

102 Càssola (1975) a.l. manca di note in merito.

103 Sul frammento si veda la più recente riflessione di Picot (2012).

104 Il dibattito è stato iniziato da Knatz (1891), che vedeva in Zeus la personificazione dell'aria e dell'etere, mentre in Aidoneus la personificazione del fuoco. Il dibattito è stato ripreso e continuato di recente da 
in cui Apollo - riconosciuto come tale nella parafrasi ammoniana del frammento ${ }^{105}$ - è ritratto con tratti solari che sarebbero poi stati dominanti nell'Inno orfico al dio (34). Potrebbero questi tratti essere già presupposti nel formulare "Apollo rapido come il pensiero" in $h A p .186,448$ ? Veloce come il vó $\mu \alpha$ è, infatti, l'Apollo che arriva sull'Olimpo in $h A p$. 186, o che incombe sulla nave dei Cretesi in $h A p$. 448; il verbo corrispettivo è inoltre usato al momento della scelta dei futuri sacerdoti, $h A p$. 391: ó@ $\mu \alpha i ́ v \omega v$

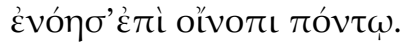

Il fr. 134 DK si inserisce all'interno di una sequenza di frammenti, inaugurata dall'invo-

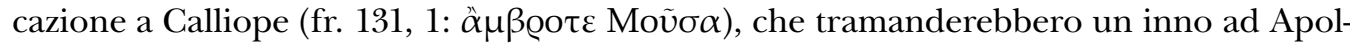
lo. Del dio vi si mostra la natura solare definita per linea paterna rispetto allo Zeus-fuoco del fr. 6 DK. E' stata avanzata, alla luce della natura e della collocazione dei frammenti nelle fonti tarde, l'ipotesi di smembramento dell'inno operato negli ambienti devoti al bios di Pitagora ed Empedocle. ${ }^{106}$ L'impiego di un vocabolario simile rafforza l'ipotesi di

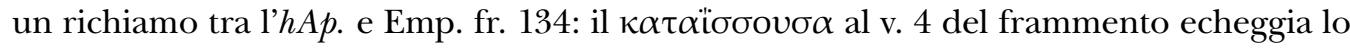

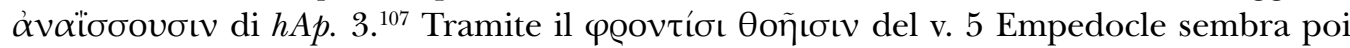
sottolineare strumentalmente la velocità di Apollo, $\varphi \varrho \eta ๋ v$ da vó $\mu \alpha$ che era in $h A p .:^{108}$ oủ

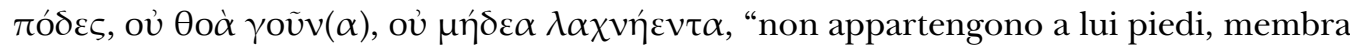

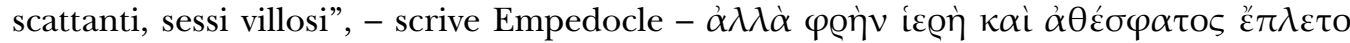

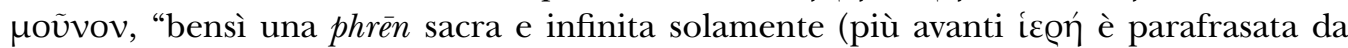
Ammonio come v́tè $\mathrm{Q}$ voũv, qualcosa che trascende l'intelletto), che in rapidi pensieri

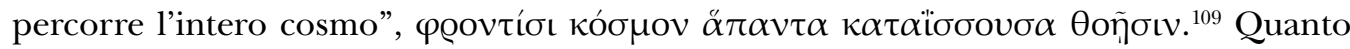
a $\varphi \varrho \eta े v$, nell' $h A p$. si ha un'elevata ricorrenza della radice $\varphi \varrho \eta v-/ \varphi \varrho o v-: ~ \varepsilon ̇ u ̈ \varphi \varrho o v \varepsilon \varsigma$ sono le Horai al v. 194, e $\varphi \varrho o v \varepsilon ́ \omega$ ricorre variamente nelle frasi rivelatrici di Apollo $(h A p .248$ :

Kingsley (1995). Per una panoramica generale, Picot (2000: pp. 25-86). Cfr. Kingsley (1995: pp. 13-35) per l'etere da lui inteso come equivalente dell'aria, senza la commistione di aria e fuoco.

105 Amm. in Aristot. De interpret. 249.1. In diversi punti il fr. 134 differisce tuttavia dalla versione di Ammonio:

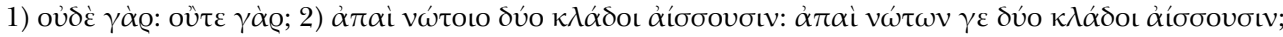

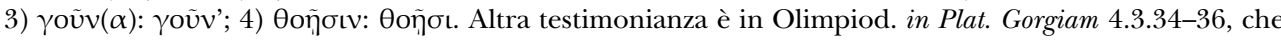
cita però solo il primo verso del frammento. L'altra testimonianza, costituita da uno scolio del Marc. Gr. 196 di Olimpiodoro, non è da prendere in considerazione: esso riporta il v. 2 tra cruces e trascrive mino-

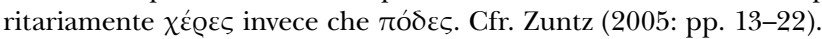

106 Solmsen (1980: pp. 219-227). Tzetz. Chiliad. XIII 79-84 K. è l'unico a restituire del fr. 134 la contestualizzazione originaria nell'opera di Empedocle. Per la storia travagliata di attribuzione del frammento, si rimanda a Primavesi (2011: pp. 446-447, 562-563).

107 Il verbo $\kappa \alpha \tau \alpha \ddot{~} \sigma \sigma \omega$ si ritrova anche nel fr. 100.7 d'Empedocle, nell'immagine della respirazione e della

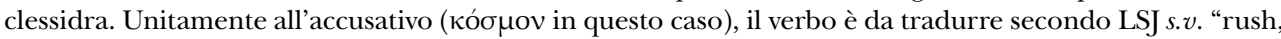
dart through".

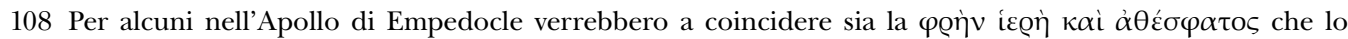
Sphairos, di cui la prima sarebbe una definizione per esteso, del fr. 29 DK. Cf. Vitek (2010); Finkelberg (1998: pp. 112-113). Si consultino anche Primavesi (2007: pp. 67-68); Palmer (2009: pp. 327-338); Drozdek (2007), che parla di un dio ma mai di Apollo.

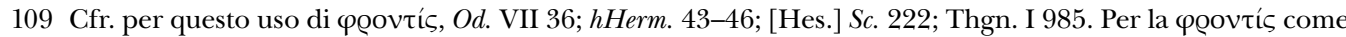
"a word with strong emotional undertones", Rangos (2012: pp. 315-328) in riferimento a Thgn. I 729, Simon. 8.9 Campbell, e Pind. O. I 19 (fonti a cui si potrebbe aggiungere anche Xen. Mem. I 4.14.7-15.1). 


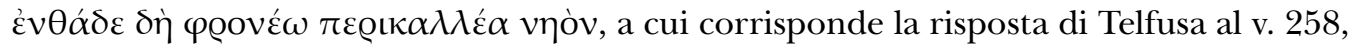

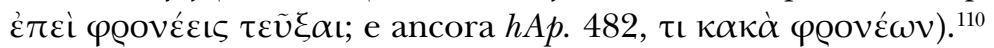

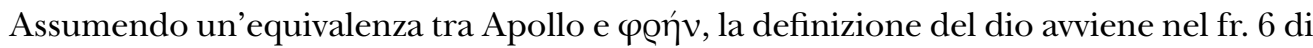
Empedocle in funzione della natura del padre nel fr. 6: dipendenza che nell' $h A p$., come si è potuto vedere, è secondaria rispetto alla personale ascesa di Apollo, e che al contrario avrà piena risonanza in Pindaro. Stabilire un legame tra Apollo e Zeus in Empedocle necessita tuttavia di un'analogia tra il secondo e l'elemento igneo primordiale. Nel fr. 6,

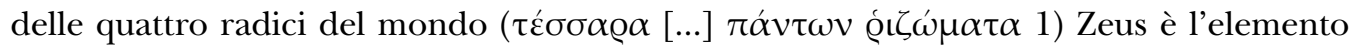

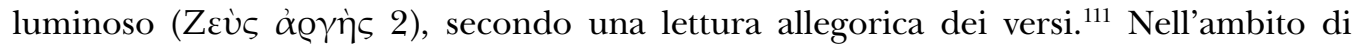
una narrazione probabilmente cosmogonica sulla spartizione divina degli spazi e degli attributi concorrente alle versioni esiodea e rapsodica, Empedocle attribuisce a Zeus, fondamento igneo del suo sistema fenomenico, un epiteto esclusivo: ג忤. Secondo Plutarco tale attributo ricorrerebbe in Empedocle per definire la pregnanza di stile tanto quanto la sostanza folgorante di una potenza divina. ${ }^{112}$ L'epiteto sottolinea, soprattutto, il passaggio dallo strumento qualificante al soggetto che manipola lo strumento stesso. Da

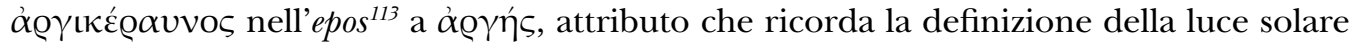
del fr. 21 ( $\dot{\varrho} \gamma \hat{\varepsilon} \tau \iota ~[\ldots] \alpha \hat{\gamma} \tilde{\eta} \iota 4)$ in un verso in cui il sole è presentato come una delle

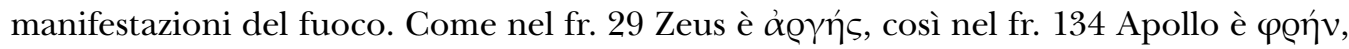
partecipe giocoforza, in quanto figlio di Zeus, dell'elemento igneo, padre di tutte le manifestazioni di luminosità e dalla natura solare. Empedocle avrebbe d'altronde operato un accostamento tra Apollo e il sole già nel fr. 47, in cui scrive che per la luna, soggetto probabilmente di $\dot{\alpha} \theta \varrho \varepsilon \tilde{\mathbf{L}}$, il sole sarebbe stato il brillante cerchio dello $\ddot{\alpha} v \alpha \xi$ ( $\alpha \hat{v} \alpha \kappa \tau o \varsigma$

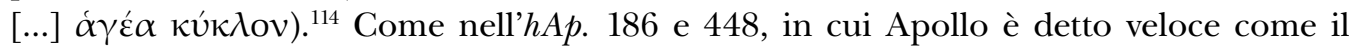

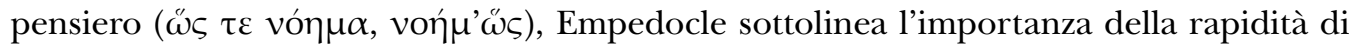

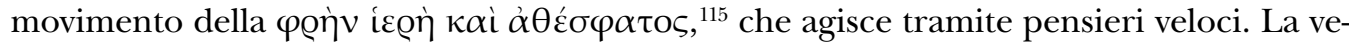
locità dei pensieri, trasposta al sole, è il movimento rapido dei raggi, che ricorda il fuoco

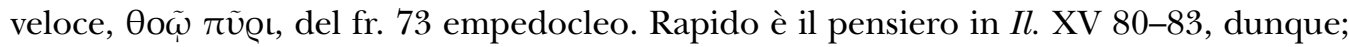

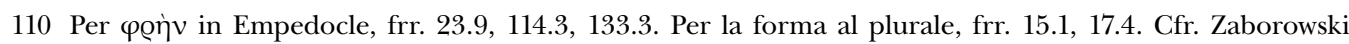
(2008: pp. 81-86). Per una sintesi di phrēn sino al V sec. a.C., Darcus Sullivan (1999: pp. 11-41). Sulle $\pi \varrho \alpha \pi i ́ \delta \varepsilon \varsigma$, Darcus Sullivan (1987: pp. 182-183). L'immagine dinamica della mente senza corpo in Empedocle ha goduto di una certa fortuna in tragedia: Aesch. Sept. 661; Soph. Ai. 447-448 con variatio di $\kappa \alpha \tau \alpha \ddot{i} \sigma \sigma \omega$ in $\alpha \pi \alpha \ddot{i} \sigma \sigma \omega$, verbo impiegato per il salto di Achille in $I l$. XXI 234. Cfr. anche Pind. O. II 57, in cui le phrenes sono come delle psychai private di corpo nell'Ade ma animate da un movimento rapido.

111 Un elemento di discordia tra gli interpreti, da cui tre diverse letture del fr. $6 \mathrm{DK}$, è stata la natura di Aidoneus, chiarita tramite tre diverse soluzioni. Per la trasmissione del motivo sino ai commentatori della tardo-antichità, si veda Journée (2012).

112 Plut. Symp. V 8.683E. Cfr. Il. VIII 133; Od. V 128; VII 249; XII 387.

113 Il. XIX 121; XX 16; XXII 178. Cfr. Arist. GC 330b 20-21; Met. 935b 1-2. Cfr. lo Zeus Keraunos di Mantinea, Cook (1928: pp. 12-13, 807).

114 Immagine presente anche Aesch. fr. 23 R., per cui Seaford (2005: pp. 602-606); Aesch. Suppl. 212-214 e Sept. 859. Per altri luoghi, si rimanda a Laurenti (1995: p. 110).

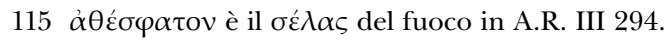


rapido come il pensiero è Apollo nell'hAp. 186, e rapide in Empedocle le phrontides di

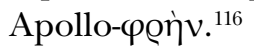

\section{L'hAp. e OrphH. 34}

L'OrphH. 34 è curiosamente diviso in due sezioni che ricalcano la struttura tendenzialmente bipartita dell'inno omerico: ${ }^{177}$ la prima incentrata sull'aspetto solare di Apollo, onorato come Menfita (1-15); la seconda dedicata alla sublimazione cosmica della solarità del dio attraverso l'argomento musicale, poi parafrasato da Claudio Tolomeo (16-25). ${ }^{118}$ I primi sette versi dell' $O r p h H .34$ non sono altro che una lista di attributi riferiti ad Apollo, di varia attinenza rispetto alle varianti del mito, ${ }^{119} \mathrm{e}$ chiusi da una participia-

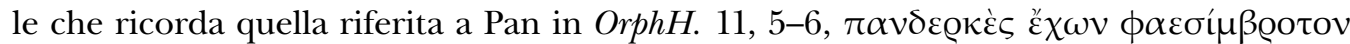
${ }_{0} \mu \mu \alpha$. L'organizzazione sintagmatica dell'epiteto negli Inni orfici è rivelatrice del funzionamento della preghiera orfica, la cui efficacia è delegata alla tecnica stessa di giustapposizione degli attributi. ${ }^{120}$ Se alcuni di questi sono di discendenza omerica e esiodea (e.g.

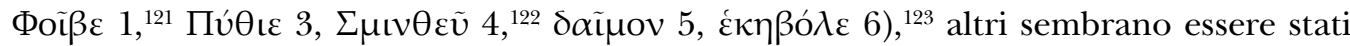

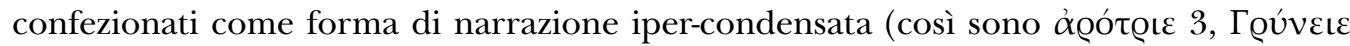

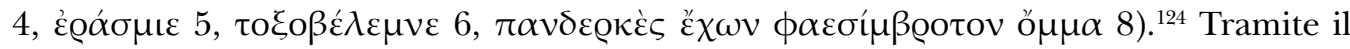
ПuӨoктóve 4 si vuole così rievocare, in un solo momento e con una sola parola, la sequenza della Pythochtonia sviluppata in extenso nell'hAp. Alcuni di questi epiteti rinviano a dei dettagli del mito assenti nell'hAp.: così il Tıtóv al v. 3, con chiaro riferimento alla sepoltura compiuta da Apollo nel Parnaso di Dioniso Zagreus straziato dai Titani. ${ }^{125} \mathrm{La}$ seconda parte dell'OrphH., dal contenuto tecnico e in cui sono adattate al contesto della

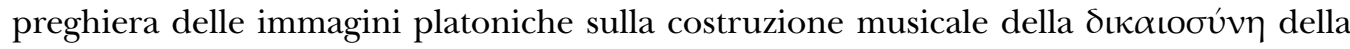

116 Com'è anche rapido il sole in Mimn. fr. 11a 1-2 ( fr. 14.11).

117 Già in Athanassakis (1977: pp. 135-136). Cfr. Fayant (2015) a.l. Quanto alla struttura dell'inno orfico,

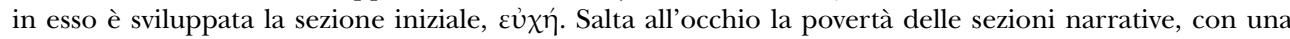
riduzione al minimo della pars epica (sic in Ausfeld 1903: pp. 505-537; argument in Bremmer 1981: n. 196) e una ricchezza eccezionale di aggettivi, participi e proposizioni relative.

118 Harm. 3.12. Sulla parafrasi del passo in Claudio Tolomeo, Solomon (2000: pp. 160-161); Barker (1989: pp. 386-387); Van der Berg (2001: pp. 168-169). Il tema dell'armonia cosmica ritorna in OrphH. 8.9, 11.6. Per il tonos dorico, Barker (1989: pp. 419-420); West (1992b: pp. 179-180).

119 Così è per il Tıтvoктóve al v. 1, che rinvia alla versione del mito per cui Leto sarebbe stata rapita da Tityos subito dopo avere partorito Apollo e Artemide (Od. II 576-581; [Apollod.] I.4.1-2).

120 Hopman-Govers (2001) ha parlato a tal riguardo di frontières de l'épithète. Sugli effetti eufonici dell'organizzazione dell'epiteto negli Inni orfici, Morand (2005). La stessa tecnica agglomerante è presente in A.P. IX 524525 per Dioniso e Apollo.

121 Anche in OrphH. 35.4, 67.6, 79.6.

122 Già in $\mathrm{Il}$. I 39.

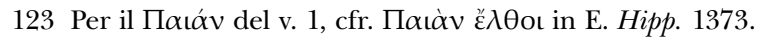

124 Cfr. Rudhardt (1991: vol. I, p. 267) per la definizione dell'epiteto orfico come di una "syntaxe (...) latente sous la parataxe".

125 Clem. Protr. II 18.1.2 = fr. 35 Kern. L'epiteto si trova nella produzione tragica riferito a Prometeo: E. Phoen. 1122; Soph. $O C 56$. Sul B $(\varrho) \alpha ́ \gamma \chi \iota \varepsilon$ del v. 7 la tradizione manoscritta non è concorde: rinvio per questo a Nisetich (2001: pp. 105, 127-129) che discute Call. frr. 194, 24-31, e 229. 


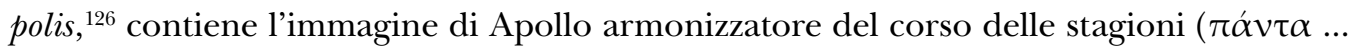

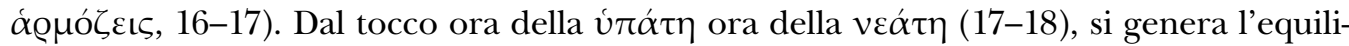

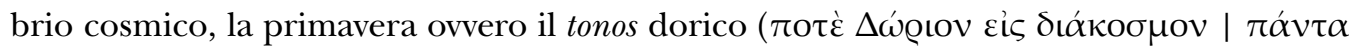

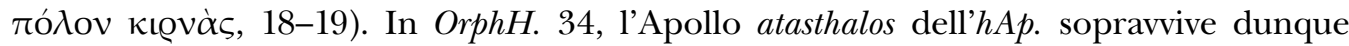
negli epiteti iniziali, che rinviano in forma condensata a episodi di uccisione dalla funzione cultuale. A queste tracce di terribilità evocata, non più narrata, si sommano aspetti demiurgici e solari, ${ }^{127}$ legati alla tecnicizzazione dell'attributo musicale del dio e recepiti dalla teoria armonica imperiale.

\section{L'hAp. e gli inni epigrafici}

Delle tre categorie di epigrafi che si prestano allo studio dell'intertestualità dell' $h A p$. epigrafi onorifiche, norme sacrali metriche, inni e peani su stele - la terza è senz'altro la più produttiva. ${ }^{128}$ È possibile rintracciare delle unità narrative principali dell' $h A p$. nei seguenti inni epigrafici: i due inni delfici, l'inno al Kouros (o Zeus) dal monte Dikta, i due peani di Aristonoo, gli inni ad Apollo e Asclepio nelle quattro versioni superstiti, e il peana di Macedonico. ${ }^{129}$ I motivi corali dell' $h A p$. sembrano essere stati quelli maggiormente recepiti dagli inni epigrafici. Insieme a questi, particolarmente frequente è il

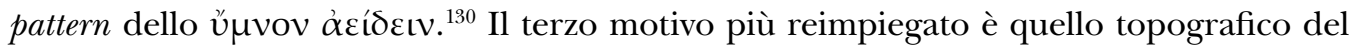
Parnaso. A seguire, l'uccisione della dracena, con un'insistenza sulla fonte Castalia, e il vaticinio dall'adyton con la sublimazione del tripode $\mu \alpha v \tau \varepsilon\llcorner\varepsilon \tilde{\imath} o v$. A differenza di quanto si è constatato nei papiri, la nascita di Apollo sembra essere stata trattata marginalmente negli inni epigrafici. Elementi isolati, ripensati appositamente per onorare Asclepio, sono invece l'unione di Apollo con Coronis, accennata in $h A p$. 208-209, e la presenza

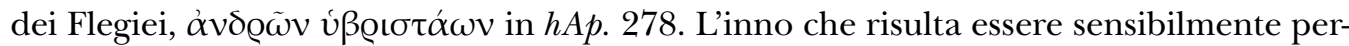
vaso di richiami all' $h A p$. è quello di Ateneo. Ad esso si dedicherà di seguito un'analisi approfondita, in cui si mostrerà come l'autore abbia costruito un coro ed un paesaggio dionisiaci attingendo direttamente a motivi dell' $h A p$. Allo stesso modo, ci si concentrerà su un paradigma performativo nuovo, quello del coro delle Delfiche, mobilitato da Ateneo per sugellare sul piano dell'evocazione poetica una nuova stagione di contatti diplomatici e religiosi tra Atene e Delfi.

126 Plat. Resp. 443d6. Sull'argomento Hagel \& Lynch (2015). Cfr. Arist. Met. 1057a 22; Probl. 919 b, 1 e $920,29$.

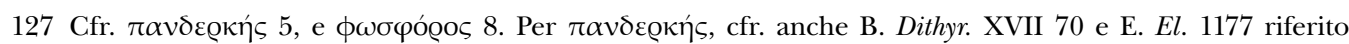

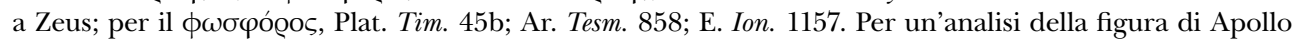
nelle comunità orfiche, Bravo (2011).

128 Per la seconda categoria delle norme sacrali e il loro legame con il retroterra prescrittivo della performance dell'hAp., si veda Colangelo (2017), con precedenti per l'Inno ad Apollo di Callimaco in Petrovic (2012) con relativa bibliografia. Riferimenti tradizionali all'esecuzione storica dell' $h A p$. sono in Aloni (1989: pp. 36-68) con un'analisi approfondita su Cineto da Chio, e West (1975). Si segnala Chappell (2011) per una sintesi aggiornata della querelle sull'unità dell'hAp., nonché Sbardella (2012).

$129 \mathrm{Ci}$ si propone di dedicare un contributo a parte alle analogie e ai riadattamenti dell' $h A p$. negli inni epigrafici, qui solo accennati in vista di una più approfondita indagine sull'inno-peana d'Ateneo.

130 Si pensi a questa formula ai vv. 7-10 dell'Inno a Zeus dal monte Dikta, in cui essa è diretta a sottolineare l'autoreferenzialità dell'inno stesso. Si veda a riguardo Furley \& Bremer (2001: vol. I, p. 60). Per l'inno del monte Dikta, cfr. Perlman (1995: pp. 161-167) e MacGillivray et al. (2000). 


\subsection{L'hAp. in Athen. Pa. 1-8}

Del frammentario inno - o per altri peana - di Ateneo, stampato per la prima volta da Weil nel 1893 e nello stesso anno commentato da Reinach prima dell'edizione di Powe1l, sono stati sino ad ora pubblicati contributi attenti al dato tecnico e ritmopoietico. ${ }^{131}$ Esso, così come l'inno di Limenio, è datato al 128/7, anno della celebrazione delle Pitiadi. Tale festività, di cui si hanno le prime notizie dal 326/5, pare si tenesse ad intervalli irregolari, con una grande ripresa nel II a.C. ${ }^{132}$ Il rilancio delle Pitiadi fu segnato da un'attiva partecipazione di Atene negli anni 138/7, 128/7, 106/5, 98/7 mediante l'invio di una nutrita theoria sacrificale composta da due exegetai, dall'intero corpo efebico, da archetheoroi e kanephoroi / pyrophoroi della Tetrapolis di Maratona, e dai technitai di Dioniso. ${ }^{133}$ Vari aspetti dell'inno di Ateneo paiono problematici se rapportati alla versione madre dell' $h A p$. - si pensi alla menzione del tripode prim'ancora dell'uccisione della

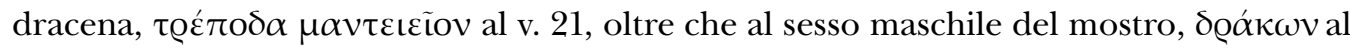
v. 22. Limitiamoci alla prima strofe dell'Inno, vv. 1-8:

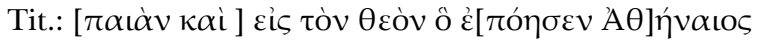

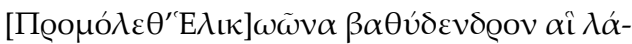

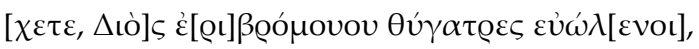

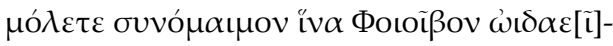

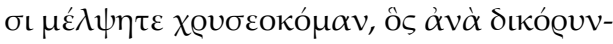

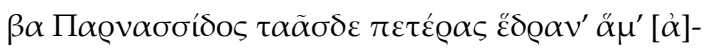

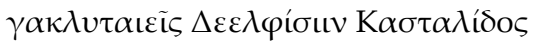

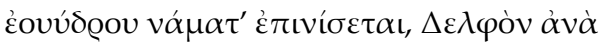

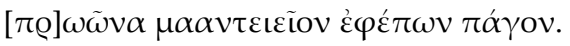

All'inizio della colonna si legge [- - $\Omega \Omega N A B A \Theta \Upsilon \Delta E N \Delta$ PNAI- $-\Lambda A[$, sequenza che introduce la prima delle molte difficoltà testuali dell'inno. ${ }^{134}$ A partire dall'impianto generale di questa prima parte di testo si può nondimeno apprezzare una suggestiva

131 Weil (1893: pp. 569-583, plate XXI 1.2); Reinach (1893: pp. 584-610); Powell (1925: pp. 141-148). Per lo studio dell'inno, Doutzaris (1934: pp. 315-340); Chailley (1979: pp. 154-158); West (1992: pp. 1-3, 16-27, 254-273); Hagel (2000: pp. 39-93). Cfr. Pöhlmann \& West (2001: p. 72) sul suo "archaic pentatonic effect".

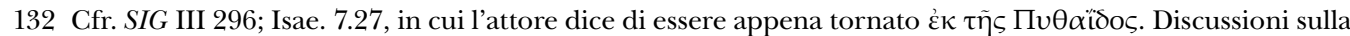
cronologia penteterica sono in Daux (1936: p. 532); Mikalson (1998: pp. 269-270); Scott (2014: p. 194). Per la cronologia degli inni, rimando a Colin (1913: pp. 529-532); Bélis (1992: p. 133); Schröder (1999: pp. 65-75); Pöhlmann \& West (2001: pp. 71-72); Furley \& Bremer (2001: vol. I, pp. 129-131).

133 Sugli esecutori di Dioniso, Le Guen (2001). Sulle sette categorie coinvolte nella theoria (otto nel caso del 128/7), Daux (1936: pp. 708-720).

134 Tutte le edizioni, anche quella più quotata di Bélis (1992), sono debitrici della prima congettura di Weil

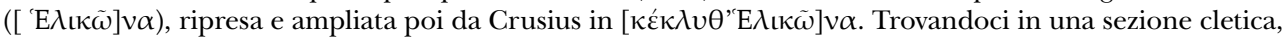
ci si aspetterebbe un $\kappa \lambda \tilde{v} \tau \varepsilon$ o un $\kappa \varepsilon ́ \kappa \lambda v \tau \varepsilon / \kappa \varepsilon ́ \kappa \lambda v \theta^{\prime}$, soluzione, quest'ultima, preferita da Crusius per

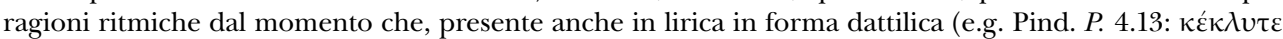

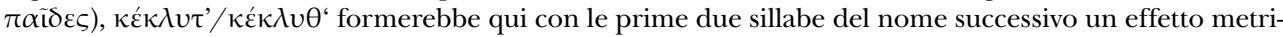
co di $5 / 8$, a conferma della vicina notazione musicale. La lezione qui riportata è tuttavia quella proposta da West nel 1992, poi abbandonata in Pöhlmann \& West con un ritorno alla lettura ottocentesca del $\kappa \varepsilon ́ \kappa \lambda v \theta^{\prime}$. Cfr. Pöhlmann \& West (2001: p. 63). 
interazione con l' $h A p$., interazione che passa dal particolare ruolo assegnato da Ateneo alle Muse, non più garanti dell'ispirazione divina ma attrici all'interno di una scena di movimento. ${ }^{135}$ Iniziando con un'invocazione alle Muse, il primo movimento dell'inno si chiude con l'arrivo del coro a Delfi e la performance dell'inno stesso, con un reimpiego da parte di Ateneo della scena olimpica delle Muse e del viaggio dei Cretesi verso Pito nell' $h A p$. Tramite questi motivi, Ateneo costruisce di Delfi l'immagine di una località di arrivo. ${ }^{136}$

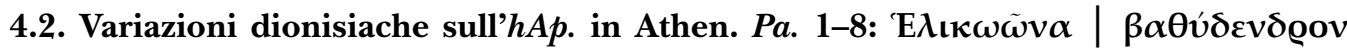 (1), ह̇[@ı]ß@ouovov (2)}

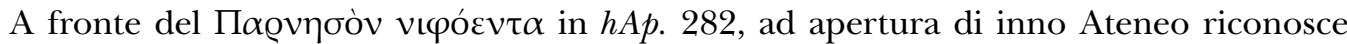
nell'Elicona il kosmos delle Muse, debitore probabilmente dell'assimilazione euripidea tra

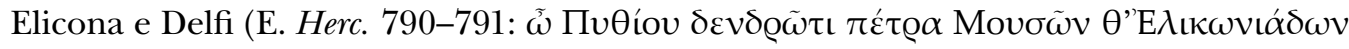

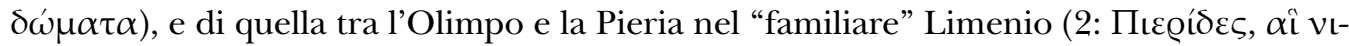

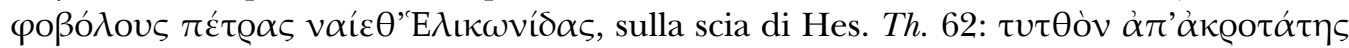

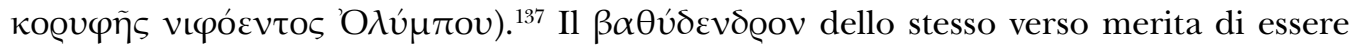
considerato. Aggettivo insolito per l'Elicona e forgiato a partire dal $\beta \alpha \theta$ v́o $\chi$ otvos usato in Il. IV 383 per l'Asopo, $\beta \alpha \theta$ v́ $\varepsilon v \delta \varrho o v$ ricorre varie volte prima di Ateneo, soprattutto

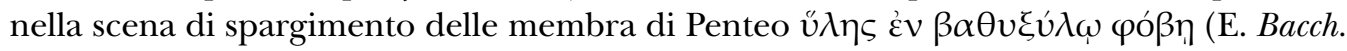
1138). Il $\beta \alpha \theta u ́ \delta \varepsilon v \delta \varrho o v$ come hapax per l'Elicona - ulteriore variazione di Ateneo rispetto all'hAp. -, se da un lato si adatta al temenos delle Muse secondo quanto Pausania dice dell'alsos dell'Elicona, ${ }^{138}$ dall'altro carica l'immagine di sfumature quasi stranianti, riprese poi da Nonno per il $\tau \varepsilon ́ \mu \varepsilon v o \varsigma ~ \beta \alpha \theta u ́ \delta \varepsilon v \delta \varrho o v$ dove le Bacchai si accampano. ${ }^{139}$ Sempre in Nonno, inoltre, l'aggettivo è associato all'uso bellico fatto da Dioniso degli alberi. ${ }^{140}$

Oltre al $\beta \alpha \theta u ́ \delta \varepsilon v \delta \varrho o v$ dell'Elicona, anche l'é[@] $\beta \varrho o \mu o v o v$ del v. 2 connota di dionisiaco la strofe. Parte di un verso gravemente compromesso nella prima parte, l'aggettivo è stato facilmente ripristinato grazie allo stato di conservazione della parte finale di

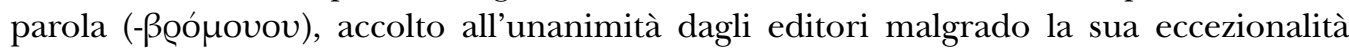
come epiteto di Zeus. ${ }^{141}$ Come già negli Inni omerici, غ̇@íßoouos ricorre per Dioniso

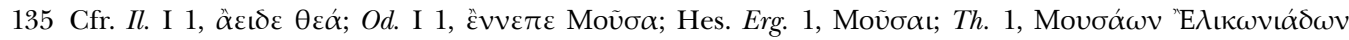

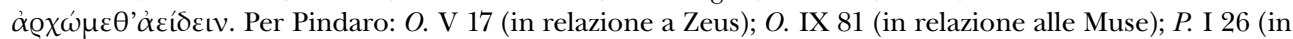
relazione a Zeus); P. IV 67 (in relazione alle Muse). Cfr. anche A.R. IV 984.

$136 \mathrm{Si}$ porti a confronto la lettura di Fontenrose su Delfi come punto di arrivo del tragitto di Dioniso: Fontenrose (1959: pp. 374-394). Per il Septerion e le feste pitiche, Fontenrose (1959: pp. 453-460). Cfr. Detienne (1977: pp. 82-83) per Dioniso mis à mort di Delfi.

137 Bélis (1992: p. 5); Furley \& Bremer (2001: vol. II, pp. 86-87). Cfr. Ibyc., fr. 17.24; Alex., fr. 9.5; Pind. I. II 34.

138 Paus. 9.29.5.

139 Nonn. D. XXVII 60. Cfr. D. XIII 184, 446; XLV 159-162. Si vedano Alcock \& Osborne (1994); Digna (2007) sull'uso dell'aggettivo per Maratona. Un caso isolato è quello in $P M G$ fr. 91, 1.1, in cui l'aggettivo è

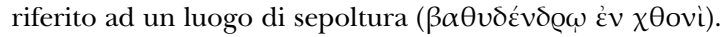

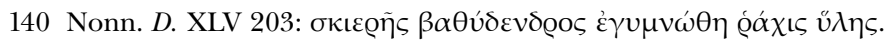

141 Weil (1893: p. 577); Fairbanks (1900: p. 122); Moens (1930: pp. 15-16). 


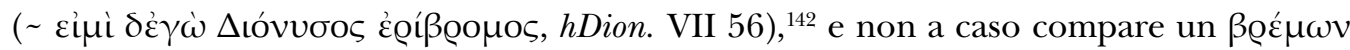
al v. 14 dell'Inno d'Ateneo riferito al $\lambda \omega \tau$ toò che sta metonimicamente per lo strumento

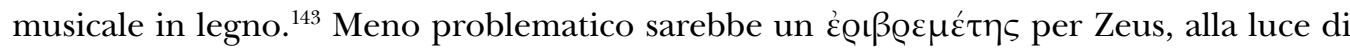

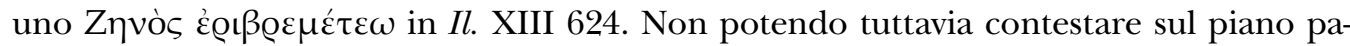

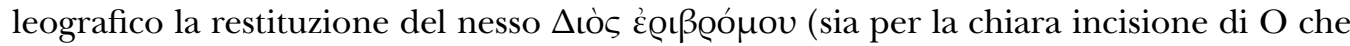
per la coerenza rispetto al $\theta \dot{\gamma} \gamma \alpha \tau \varrho \varepsilon \varsigma \varepsilon \dot{u} \omega \dot{\lambda}[\varepsilon v o l]$ del v. 3), ${ }^{144}$ una soluzione per interpretare l'innovazione d'Ateneo consisterebbe a mio avviso nel supporre una sovrappo-

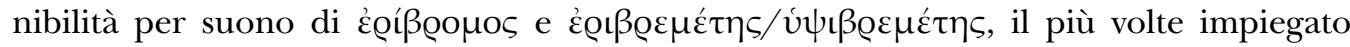
quest'ultimo per Zeus, con una variazione nello $\beta \alpha \varrho \mho \beta \varrho \varepsilon \mu \varepsilon ́ \tau \alpha$ di Soph. Antig. $1116 .{ }^{145}$

\subsection{Variazioni cletico-cinetiche sull'hAp. in Ath. Pa. 1-8: $\pi \varrho o \mu o ́ \lambda \varepsilon \theta^{\prime}$ (1), ív $\alpha$...

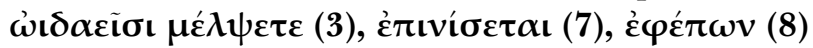

La connotazione dionisiaca di un paesaggio per tradizione apollineo non è il solo aspetto da considerare per questa prima strofe dell'inno di Ateneo. Ci si concentri sull'invocazione alle Muse in esso contenuta, ad esempio.

L'invocazione alle Muse non è un dato scontato nella tradizione innografica. Sui trentatré inni della collezione omerica, solo cinque ne contengono una: oltre all'hHerm. 1-2 et all'hAphr. 1-2, solo l'hHelios presenta un iniziale Moṽ $\alpha \alpha$ K $\alpha \lambda \lambda$ เó $\eta \eta$, e gli $h S e l$. e $h D i o-$ sc. un plurale Moṽ $\sigma \alpha \mathrm{t} .{ }^{146}$ Fatto ancora più rilevante, gli inni ad Apollo mancano dell'invocazione alle Muse come elemento normativo e formulare, ad eccezione dell'hHom. XXV

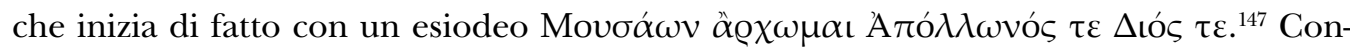
siderando l' $h A p$. come un insieme quadripartito, ${ }^{148}$ molteplici sarebbero stati i momenti per invocare le Muse. Esse compaiono al contrario a due riprese e con funzioni diverse da quelle cletiche: in $h A p$. 189, come coro divino che assiste al secondo accesso di Apollo nell'Olimpo; in $h A p$. 516-518, come modello dei Cretesi intonanti il peana.

142 Eccezionalmente per Efesto in Trifodoro, 232: 'H

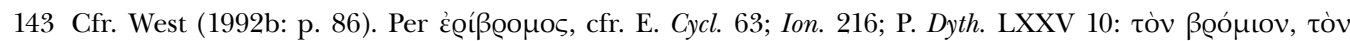

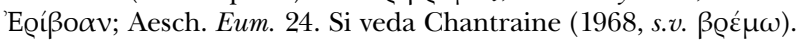

144 Anche in merito al $\theta \hat{\gamma} \gamma \alpha \tau \varrho \varepsilon \varsigma \varepsilon \dot{v} \omega \dot{ } \lambda[\varepsilon v o t]$ vorrei sottolineare l'eccezionalità del definire le Muse $\Delta$ tò $\varsigma .$.

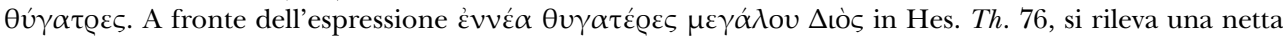

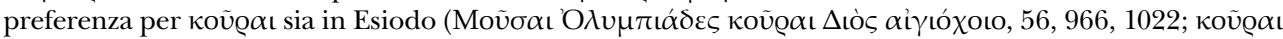

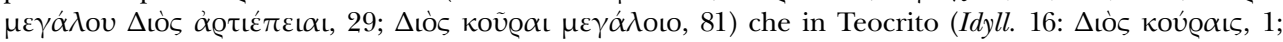

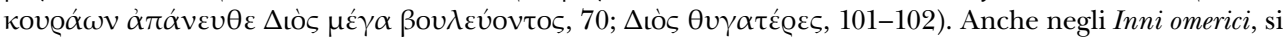

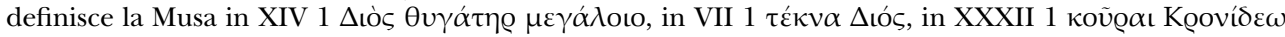
$\Delta$ ıós.

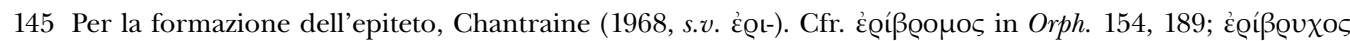

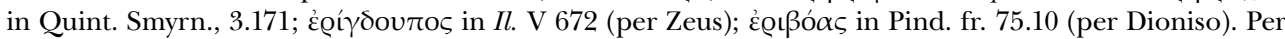

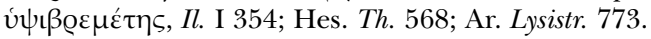

146 Per l'hHerm., Vergados (2013: p. 218). Per hAphr., Olson (2012: p. 129). Altre occorrenze dell'invocazione in Il. XI 218 = XIV 508 = XVI 112 = Il. Cyc. 1.1, 64 Bernabé; adesp. PMG 938 (c); Alcm. PMG 27.1; Hippon. fr. 128.1 Degani.

147 Per l'assenza di un'invocazione alle Muse nel Peana di Aristonico, Powell (1925: pp. 162-164), Käppel (1992: p. 384), West (1992b: p. 139).

148 I due prooimia sul parto a Delo, vv. 1-146, e la ricerca dell'oracolo, vv. 179-513; il peana narrativo o appendice pitica, vv. 514-546; il frame della performance delia, vv. 147-178. Cfr. il più recente modello tripartito in Richardson (2010: pp. 9-13), e quello in Esteban Santos (1981). 
Nel suo inno, Ateneo si rivolge alle Muse con un $\pi \varrho 0 \mu o ́ \lambda \varepsilon \theta$ ', sì da incitare il coro a muoversi verso Delfi. A fronte del $\kappa \varepsilon ́ \kappa \lambda v \theta$ ' congetturato da West (1992) e accolto da Hagel (2000), ${ }^{149}$ credo che la restituzione del $\pi \varrho 0 \mu o ́ \lambda \varepsilon \theta$ ' sia qui da preferire, dato il succedere del $\mu$ ó $\lambda \varepsilon \tau \varepsilon$ al v. $3,{ }^{150}$ con una ripetizione del comando volta a riattualizzare il motivo principale, Apollo / le Muse verso Delfi. Si consideri inoltre la particolarità del verbo, che indicherebbe molto più del semplice "muovere verso", espresso dal

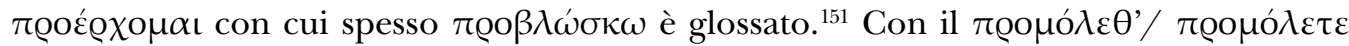
il poeta intenderebbe piuttosto enfatizzare il momento iniziale della presa di movimento. A ciò si aggiunge l'eccezionalità dell'impiego di $\pi \varrho o \mu o ́ \lambda \varepsilon \tau \varepsilon$ in riferimento ad una divinità in contesto cletico. ${ }^{152}$ L'intento del poeta sembra essere stato quello di marcare, anche in chiave cletica, lo statuto per Delfi di luogo di arrivo, variando in tal modo sul terzo movimento dell' $h A p$. incentrato sulla marcia di Apollo in direzione dell'oracolo. Tale doveva essere Delfi per i mortali della theoria ateniese del 127 a.C. e per il coro delle Muse, visitatrici dirette dell'oracolo. Pertanto ho ritenuto opportuno riportare nel testo la congettura $\delta \varepsilon \tilde{v} \varrho ' \imath \bullet$ all'imperativo. $\mathrm{E}$ in tale direzione si dovrà ugualmente interpretare l'c่ $\tau \iota v i ́ \sigma \varepsilon \tau \alpha \iota$ del v.

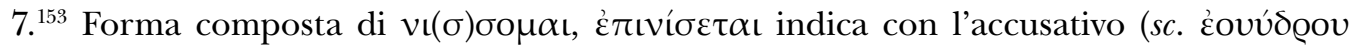
$v \alpha ́ \mu \alpha \tau^{\prime}$, le acqua di Castalia) il "viaggiare verso", con enfasi posta sul momento dell'arrivo o della visita. ${ }^{154}$ In tal modo si riaffermerebbe a fine della strofe il motivo dello spostamento verso Delfi, luogo dell'arrivo, della vittoria sulla dracena, della Pythochtonia, della performance celebrativa. Proprio a chiusura del movimento, troviamo, riferito all'arrivo di Apollo a Delfi, દ̇ $\varphi \dot{\tau} \tau \omega \nu$, anch'esso enfatico rispetto al telos del viaggio se si

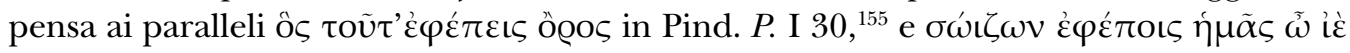
$\Pi \alpha i ́ \alpha v$ al v. 47 del peana di Aristonico, in cui il telos del viaggio viene significativamente a coincidere con l'epifania di Apollo.

La caratterizzazione delle Muse come coro dionisiaco a Delfi continua con lo iv $\alpha$... $\omega \iota \delta \alpha \varepsilon \tilde{\imath} \sigma \iota \mu \varepsilon \dot{\varepsilon} \psi \varepsilon \varepsilon \tau \varepsilon$ al v. 3: nesso che, oltre a riecheggiare $h A p$. 189, riprende l'hHer. 476

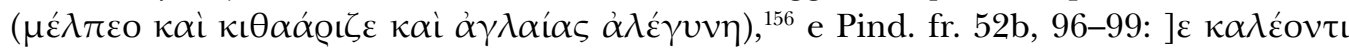

149 Contra Pöhlmann \& West (2001 a.l.). Cfr. Moens (1930: p. 14); Bélis (1992: p. 58); Furley \& Bremer (2001: vol. II, pp. 86-87).

150 Moens (1930: p. 16); Furley \& Bremer (2001: vol. II, p. 87); West (1990: p. 96).

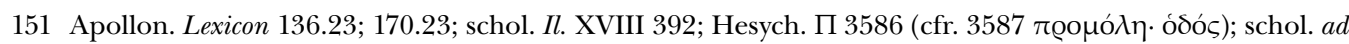
A.R. IV 523.

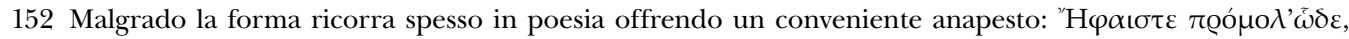

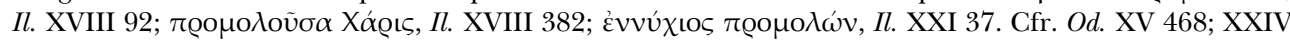

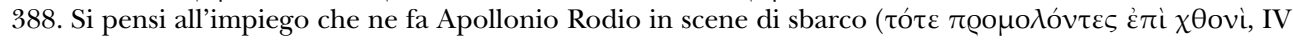
523) e a quello di Callimaco, nell'inno a Artemide, per indicare l'aggirarsi della dea tra i campi. Cfr. The-

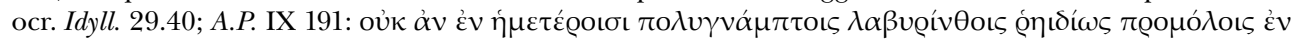

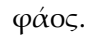

153 Furley \& Bremer (2001: vol. II, p. 88).

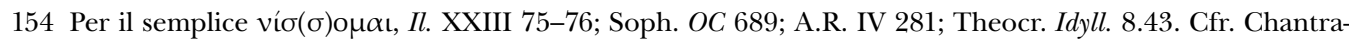

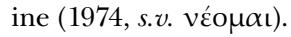

155 Verbo che indica l'imbattersi nella morte in $I l$. VII 52, XXI 100.

156 Cfr. Vergados (2013 a.l.); Moens (1930: pp. 16-17). Si pensi anche a Il. XVIII 603; Od. IV 17; E. Tr. 547. 


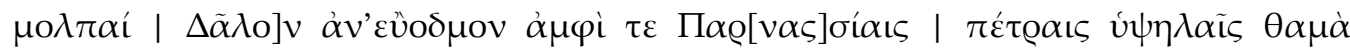

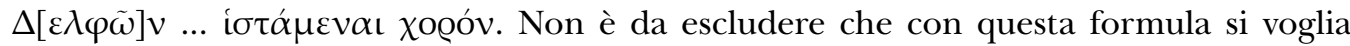
inoltre dionisizzare le Muse in memoria del culto di Dioniso Melpomenos nella casa di Polytion ad Atene, ${ }^{157}$ al quale era votato il coro dei technitai ateniesi. ${ }^{158}$

\subsection{Variazioni attributive dell'hAp. in Ath. Pa. 1-8: $\varepsilon \dot{v} \omega \dot{\lambda}$ [عvot] (2)}

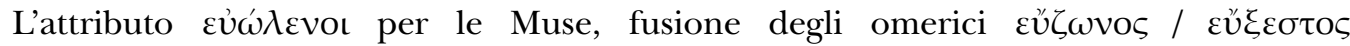

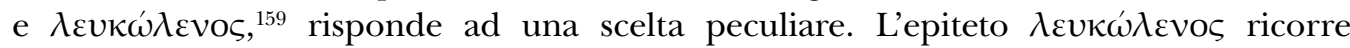
nell'hAp. 95 per Era, raramente altrove per le Muse ${ }^{160}$ salvo in Bacchilide per Calliope

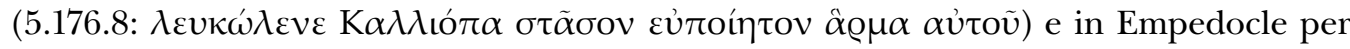

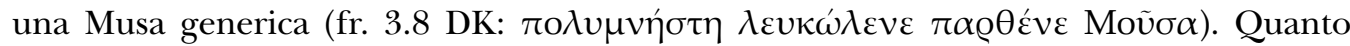

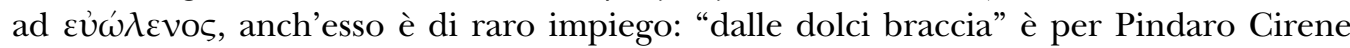

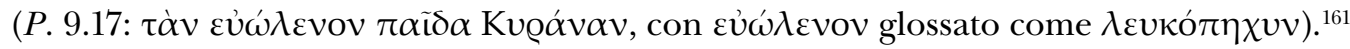

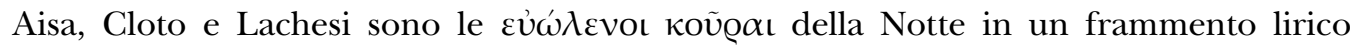
( $P M G$, fr. 100b 1.1), ed Euripide impiega l'aggettivo per descrivere il braccio di Ippolito (Hipp. 606: $\tau \tilde{\eta} \sigma \delta \varepsilon \delta \varepsilon \xi \tilde{\alpha} \varsigma \varepsilon \dot{u} \omega \lambda \varepsilon \dot{v} v o v$ ). In un frammento esiodeo si narra inoltre del

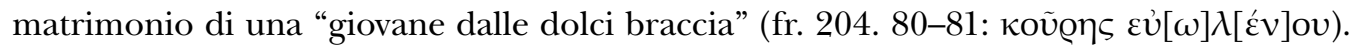
Alla luce di quanto detto, la qualificazione delle Muse in Ateneo sembra doppiamente

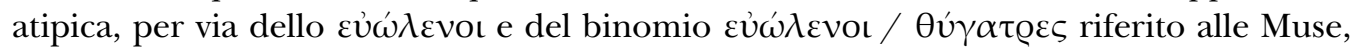
alle quali spettano solitamente epiteti geografici o qualificativi rispetto al canto. ${ }^{162}$ Così è d'altronde in $h A p$. 189-194, dove le Muse ò $\pi \grave{i} \kappa \alpha \lambda \tilde{n}$ cantano inni con le Charites

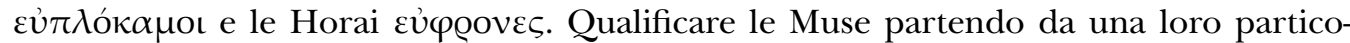
larità o attitudine fisica risponde in Ateneo alla volontà di accentuare, partendo dalla figurazione neutra del coro nel corteo peanico nell' $h A p$., la partecipazione orchestrale delle Muse allo spostamento verso Delfi. Le Muse sono questa volta, in Ateneo, attrici.

Configurando questo complesso divino, Ateneo persiste nel colorare di dionisiaco gli

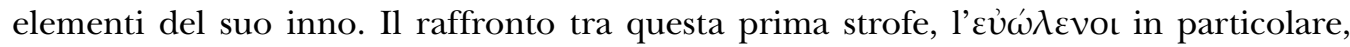
e E. Bacch. 1202-1207 è in tal senso illuminante. Nei versi della tragedia si invocano le

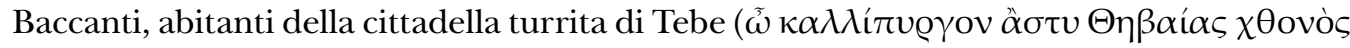

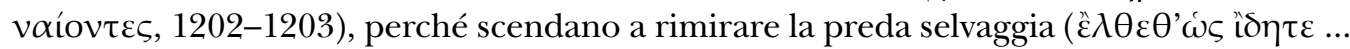

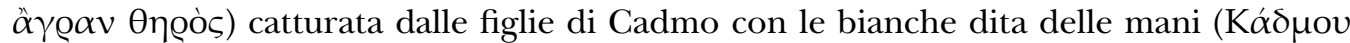

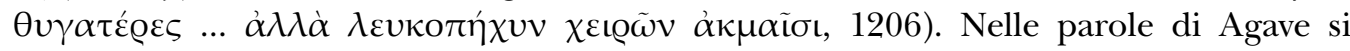

157 Paus. I.2.5.

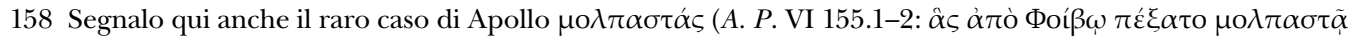

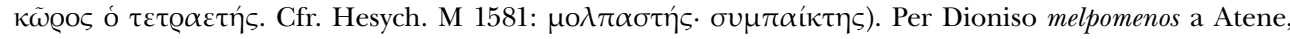
culto essenzialmente privato, Kerényi (1976: p. 161, n. 88). Si rinvia inoltre a Haspels (1936, pl. XXVIII 2) per il Dioniso Melpomenos onorato nel demo d'Acarni insieme a Dioniso Kissos. Cfr. da ultimo Stratiki (2013: p. 410).

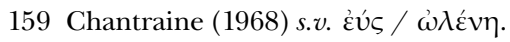

$160 \lambda \varepsilon v \kappa \omega ́ \lambda \varepsilon v o \varsigma$ anche in Il. I 55; VI 371 (per Andromaca); Od. VI 101 (per Nausicaa); VII 233 (per Arete).

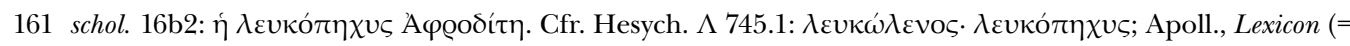

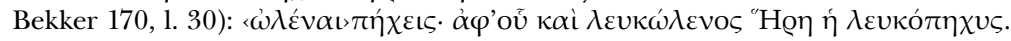

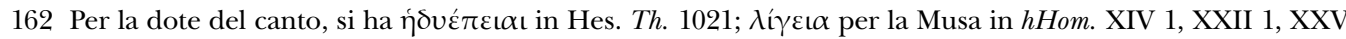

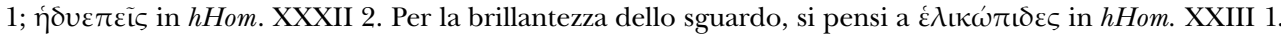


nascondo gli stessi tre elementi che strutturano la partecipazione delle Muse nella sezione cletica di Ateneo, che innova attraverso il coro euripideo delle Bacchai il modello neutro delle Muse dell'hAp.: ipotesi confortata dal fr. 752 di Euripide, in cui Dioniso è

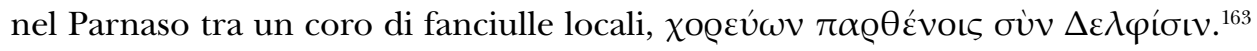

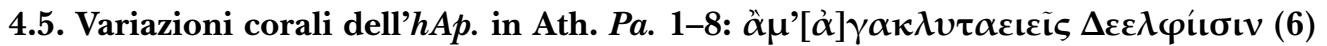
Come il coro delle Deliadi nell' $h A p$., così le donne di Delfi partecipano nell'inno di Ateneo alla performance e alla danza marciante, controbilanciando così l'azione delle Muse sinora descritta.

Il nesso $\ddot{\alpha} \mu$ '[ $\alpha] \gamma \alpha \kappa \lambda v \tau \alpha \varepsilon \iota \varepsilon \tilde{\iota} \varsigma \Delta \varepsilon \varepsilon \lambda \varphi$ í $\sigma \iota v$ del v. 6 ha variamente suscitato l'interesse degli editori, soprattutto per il $\Delta \varepsilon \varepsilon \lambda \varphi$ ú $\sigma \iota v .{ }^{164}$ Se per Crusius esso si riferisce ad un gruppo di sacerdotesse addette al sacrificio e agli oracoli di Apollo, ${ }^{165}$ per Moens e questa sarebbe l'ipotesi più credibile stando alle fonti - alluderebbe piuttosto ad un gruppo locale di donne. Questo, investito dello stesso ruolo delle Deliadi nell'hAp.,

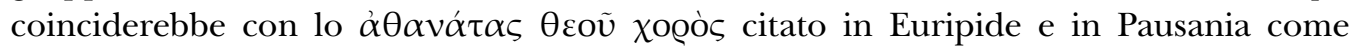
sacro ad Apollo e Dioniso. ${ }^{166}$ Due soluzioni si prospettano a mio avviso per spiegare

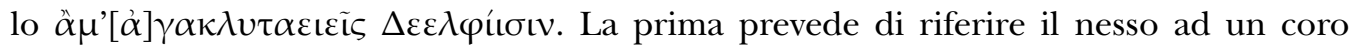
di mortali che, carico anch'esso di nuances dionisiache, impersonerebbe ad un diverso livello di performance le Muse della stessa strofe. La seconda permetterebbe di cogliere la volontà d'Ateneo di offrire tramite le Delfiche un parallelo al canto delle Deliadi,

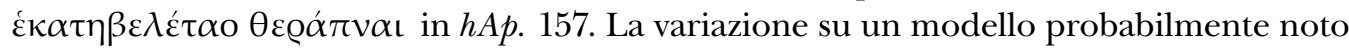
ai partecipanti alle Pitiadi si giustificherebbe come strategia poetica volta a coronare performativamente un momento di significativo contatto politico tra Atene e Delfi, proprio come la sphragis dell' $h A p$. 156-164, pensata per sugellare il nascente dominio di Atene su Delo sotto i Pisistratidi. ${ }^{167}$

163 Cfr. Nonn., D. XIII 129-131, in cui Apollo condivide il temenos di Delfi con Dioniso $\kappa \alpha \sigma \gamma \gamma v \dot{\tau} \tau$. Cfr.

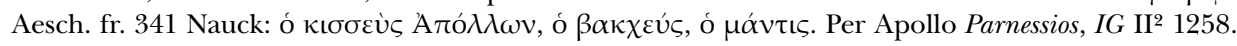

164 Weil (1893: p. 577); Crusius (1894: pp. 41-42); Fairbanks (1900: p. 122); Moens (1930: pp. 18-19); Furley \& Bremer (2001: p. 87). L'aggettivo [a்] $\gamma \alpha \kappa \lambda v \tau \alpha \varepsilon เ \varepsilon \tilde{\iota} \varsigma$, chiaramente connesso con la sfera del kleos, compare già per Efesto e Hygeia in Hes. Th. 945 e nel peana d'Eritre, v. 14. Se ne trova una spiegazione in Hesych., s.v. $\dot{\alpha} \gamma \alpha \kappa \lambda v \tau \alpha \dot{\alpha}$.

165 FD III, 2. no. 29, 30, 26.

166 E. Phoen. 234-238; Paus. 10 32.7. Sulle Thyades, Kerényi (1976: p. 218).

167 Questa nuova stagione di contatti tra Atene e Delfi sarebbe riflessa in diversi documenti tra il 170-90 a.C. Ne propongo qui una lista: (i) un decreto onorifico per Apollodoro d'Atene del 168/7 (Nachtergael [1977: p. 454] = inv. 730. Cfr. Colin [1905: p. 165, n. 66, epigr. 39] = BCH 30 [1906: p. 326, n. 66]); (ii) due decreti onorifici per i technitai del 106/5 e del 98/7 (inv. 306, 316 [Colin 1905: p. 116, n. 50] = BCH 30 [1906: p. 275, n. 50]; inv. 100, 476, 1458, 1575, 4574 [Colin 1905: p. 114, n. 49] = BCH 30 [1906: p. 273, n. 49]);

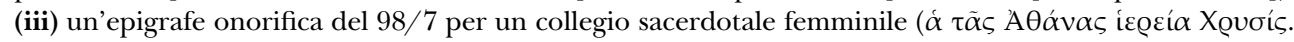
$F D$ III, 2 no. 20 = CIA II 550. Cfr. FD III 2 no. 48, ll. 8-10 sulla necessità per il bene di mogli, figli, amici e alleati di inviare la theoria a Delfi); (iv) un testo sull'arbitrato di Atene nel 140 per la ridefinizione dei confini della hiera chora di Apollo tra Delfi e Phlygonion-Ambryssos (Rousset [2002: pp. 126-127], inscr. 3 = FD III 2, 136, pl. IV 2); (v) una lista di beneficiari ateniesi di enktesis a Delfi nel 167/6 sino al 106/5 (FD III 2, 91; BCH 66/7 p. 130; Syll. 3711K). 


\section{Conclusioni}

Nel presente contributo si è voluto porre delle prime basi per un'analisi del quadro intertestuale dell' $h A p$., che meriterà senza dubbio di essere estesa ad altri testi, qui ignorati o solo velocemente evocati. L'indagine si è fondata principalmente sul reperimento di motivi che, presenti nell' $h A p$., sono stati a vari livelli soggetti a trasposizione in un numero circoscritto di testi prevalentemente su papiro e epigrafe. I casi discussi hanno permesso di rintracciare in ogni singolo testo diversi registri di allusività al modello dell' $h A p$., nelle strutture narrative così come in singoli motivi e formule. Varie tecniche di allusione risultano essere state mobilitate dagli autori per rimodellare i motivi dell' $h A p$. Si può supporre che un pubblico di fruitori abbia dovuto quantomeno posizionarsi rispetto al testo a vari livelli di familiarità, riconoscendone dunque passaggi ed eventualmente il loro succedersi, singoli motivi e schemi metrico-formulari.

L'indagine intertestuale non può senz'altro considerarsi un mezzo sufficiente per stabilire una cronologia relativa dell' $h A p$., restando la produzione innica preesistente ad esso pressoché ignota. D’altronde, "the real difficulty with allusion or intertextuality in early Greek hexameter poetry is not one of theory but of evidence". ${ }^{168}$ Lo scopo del contributo non è stato pertanto voler individuare un contesto primario o secondario di uso e riadattamento di un determinato motivo, bensì fornire un primo quadro della presenza dell' $h A p$. in documenti contemporanei e successivi alla sua ricezione. La ricerca di una cronologia relativa dei singoli motivi ('motivgeschichtliche Forschung') è uno degli aspetti che, conseguenti alla presente riflessione, potranno essere in futuro trattati sì da arricchire e correggere la ricostruzione qui proposta. Il caveat di Currie dovrà in tal senso guidare ogni indagine futura, giacché “(...) a relative chronology of motifs does not equate to a relative chronology of the poems in which they occurr". ${ }^{169}$

Si propone a conclusione del contributo lo stemma di riferimento per la rete intertestuale dell' $h A p$. sinora discussa, approntato a seguito di un'analisi svolta su testi di per sé controversi. ${ }^{170}$ Nello stemma che segue, frutto di una ricostruzione ipotetica che potrà in futuro essere riconsiderata ed emendata alla luce di ulteriori letture critiche, si intende giustificare in via congetturale i rapporti di dipendenza o di plausibile relazione tra l'hAp. $(x)$ e i testi menzionati e, in alcuni casi, commentati $(y)$. Si ricorre pertanto alla linea continua in caso di discendenza o di contaminazione più o meno certe tra l'hAp. e $y$, alla linea tratteggiata per le relazioni dubbie ma intertestualmente plausibili. Emergeranno così nitidamente delle linee in grado di circoscrivere idealmente il raggio della preistoria, della fortuna e soprattutto del rimpiego dell' $h A p$. dal VII secolo sino al III d.C. Allo stesso modo saranno più immediatamente visibili i rapporti tra $x$ stesso e le diverse "famiglie" del mito, ricostruiti secondo il ricorrere variato di sette costanti: (a) la patrilinearità nella definizione del potere di Apollo; (b) la nascita di Apollo a Delo; (c) l'epiteto di Apollo; (d) le tracce di simbologia geografica; (e) la Pythochtonia;

168 Dowden (1996: p. 48) e Currie (2012: p. 208).

169 Cfr. Currie (2012: p. 206).

170 Cfr. il modello di Vergados (2013: pp. 76-124) per l'hHerm. 
(f) l'onniscienza solare di Apollo; (g) la funzione mimetica delle Deliadi. A tali costanti si sono aggiunti, per gli inni epigrafici, (i) le scene corali, (ii) lo v̌́ Parnaso, (iv) l'unione di Apollo con Coronis, (v) i Flegiei. Di seguito, i principali testi discussi e di cui si è cercato di individuare il grado di parentela rispetto all'hAp.: ${ }^{171}$

Alcae. Hymn. Ap. (= Him. Or. 14); Stes. PMGF 222b, 204-210; Hymn. Hom. Herm.; Hymn. Hom. Dion.; Pind. Pa. XII (P.Oxy 1792); Pind. Pa. VI (P. Oxy. 841; PSI 147); Pind. Pa. VIIb (P. Oxy. 2440); Emp. fr. 134 DK; P. Louvre E7734 (+ 7733); SEG 9.72 = LSS 15; Call. Hymn. II; Call. Hymn. IV; Call. Ai. III 2-3; (P. Oxy 2212); Isyll. Pa.; Philod. Pa. Hest. Ap.; Philod. Pa. Dion.; Athen. Hymn. Ap.; Limen. Hymn. Ap.; P. Yale 1187; P. Lit. Goodspeed 2; Hymn. Orph. XXXVI; PMG III (= P. Louvre 2396); PMG VI (= P. Par. Bibli. 574); P. Herc. 1088/433, 13-22; Claud. Ptol. Harm. 3.12; SGO 1.01.19.01; SGO 1.02.02.01.

\section{Intertestualità dell'hAp.: uno stemma}

Preesistenti inni in esametri?

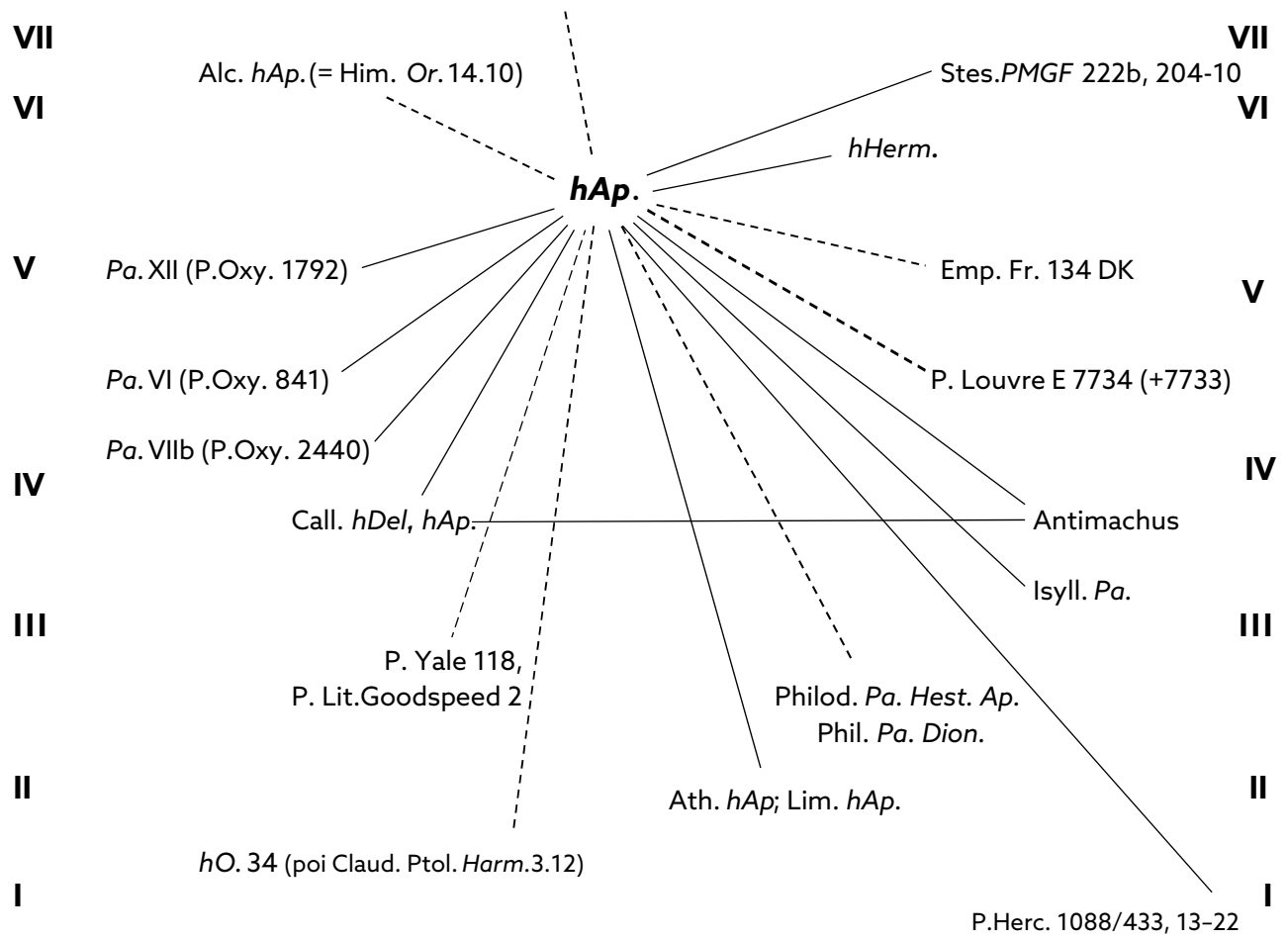

171 Sono stati volutamente esclusi dalla seguente lista e dallo stemma i testi paralleli di volta in volta citati nel corpo del testo o in nota. 


\section{Bibliografia}

Alcock, S., \& Osborne, R. (1994). Placing the Gods: Sanctuaries and Sacred Space in Ancient Greece. Oxford: University Press.

Allen, T. W., Halliday, W. R., \& Sikes, E. E. $\left(1936=1963^{2}\right)$. The Homeric Hymns. Oxford: University Press.

Aloni, A. (1989). L'aedo e i tiranni. Ricerche sull'Inno omerico ad Apollo. Roma: Ed. dell'Ateneo.

Aloni, A. (2004). L'ira di Era: tracce di committenza samia nell'Inno omerico ad Apollo. In E. Cavallini (Ed.), Samo: storia, letteratura, scienza. Atti delle giornate di studio (Ravenna, 14-16 novembre 2002) (pp. 13-29). Pisa - Roma: Istituti editoriali e poligrafici internazionali.

Aloni, A. (2009). The Politics of Composition and Performance of the Homeric Hymn to Apollo. In L. Athanassaki, R. P. Martin, \& J. Miller (Eds.), Apolline Politics and Poetics (pp. 55-63). Athens: European Cultural Centre in Delphi.

Ancher, G. (1978). P. Lille 111 c et P. Lille 76 abc (+ 73). Zeitschrift für Papyrologie und Epigraphik, 30, 27-35.

Ancher, G., Boyaval, B., \& Meillier, C. (1976). Stésichore (?) (P. L. 76abc). Les Cahiers de Recherches de l'Institut de Papyrologie et d'Égyptologie de Lille, 4, 287-351.

Arrighetti, G. (2003). Filodemo biografo dei filosofi e le forme dell'erudizione. Cronache Ercolanesi, 33, 13-30.

Athanassakis, A. N. (1977). The Orphic Hymns. Missoula: Scholars Press for the Society of Biblical Literature.

Ausfeld, K. (1903). De Graecorum precationibus quaestionibus. Jahrbuch für classische Philologie, 28, 502-547.

Barbantani, S. (2005). Goddess of Love and Mistress of the Sea. Notes on a Hellenistic Hymn to Arsinoe-Aphrodite (P. Lit. Goodsp. 2, I-IV). Ancient Society, 35, 135-165.

Barbantani, S. (2005b). L'inno ad Afrodite-Arsinoe in P. Goodspeed 101. In R. Pretagostini, \& E. Dettori (Eds.), La cultura ellenistica. L'opera letteraria e l'esegesi antica. Atti del Convegno COFIN 2001, Università di Roma Tor Vergata, 22-24 settembre 2003 (pp. 135-153). Roma: Edizioni Quasar.

Barbantani, S. (2008). Some Remarks on P. Goodspeed 10 and the Ortography of the "Ptolemaic Hymns". P.Lit.Goodspeed 2. In E. Cingano, \& L. Milano (Eds.), Papers on Ancient Literatures: Greece, Rome and the Evidence of the Near East. Proceedings of the Venice International University 2004-2005 (pp. 1-32). Padova: S.A.R.G.O.N. Editrice e Libreria.

Barker, A. (1989). Greek Musical Writings (Vol. 2). Cambridge: University Press.

Bélis, A. (1992). Les deux hymnes delphiques à Apollon. Étude épigraphique et musicale. Paris: De Boccard.

Bernabé, A. (1987). Poetae Epici Graeci (Vol. I). Leipzig: Teubner.

Bernardini, P. (2009). L'Inno primo di Pindaro e la sua destinazione cultuale. Paideia, 64, 73-89.

Bernardini, P. (2012). Cos e i Meropi nel peana epico Meropis: una possibile ricostruzione. In

G. Cerri, A. T. Cozzoli, \& M. Giuseppetti (Eds.), Tradizioni mitiche locali nell'epica greca. Atti del convegno di studi in onore di A. Martina (pp. 180-189). Roma: Scienze e Lettere.

Bierl, A., Lämmle, R., \& Wesselmann, K. (2007). Literatur und Religion. Wege zu einer mythischrituellen Poetik bei den Griechen (2 Vols.). Berlin - New York: de Gruyter.

Blass, F. (Ed.). (1917). Hyperidis Orationes sex cum ceterarum fragmentis. Lipsiae: Teubner. 
Blumenthal, A. von (1942). Paian. In G. Wissowa et al. (Eds.), Paulys Realenzyklopädie der classischen Altertumswissenschaft (Vol. 18; coll. 2340-2362). Stuttgart: Metzler.

Boisacq, E. (1916). Dictionnaire étymologique de la langue grecque. Heidelberg: Winter.

Bollack, J., Judet de la Combe, P., \& Wismann, H. (1977). La réplique de Jocaste. Papyrus Lille 73 et 76 abc. Supplément au Cahier de Philologie, 2, 1-17.

Bona, G. (1988). Pindaro: I Peani. Cuneo: Saste.

Bonanno, M.G. (1990). L'allusione necessaria. Ricerche intertestuali sulla poesia greca. Roma: Edizioni dell'Ateneo.

Boserup, I. (1971). Zu Philodems De Pietate und Heraklit B80. Zeitschrift für Papyrologie und Epigraphik, 8, 109-115.

Bowie, E. L. (2010). Historical Narrative in Archaic and Early Classical Greek Elegy. In D. Konstan, \& K. A. Raaflaub (Eds.), Epic and History (pp. 145-166). Chichester - Malden, MA: Wiley-Blackwell.

Bravo, B. (2011). Una tavoletta d'osso da Olbia Pontica della seconda metà del 6 secolo a.C. (SEG 36, 694): Apollo di Didyma e la nascita di Olbia polis. Zeitschrift für Papyrologie und Epigraphik, 176, 99-119.

Breglia Pulci Doria, L. (1996). La Meropis epica e la "Meropis" di Teopompo: proposte di lettura. In C. Montepaone (Ed.), L'incidenza dell'antico. Studi in memoria di Ettore Lepore (Vol. 3; pp. 385-398). Napoli: Luciano.

Brelich, A. (1958). Gli eroi greci. Un problema storico-religioso. Roma: Ed. dell'Ateneo.

Bremmer, J. M. (1981). Greek hymns. In H. S. Versnel (Ed.), Faith, hope and worship. Aspects of Religious Mentality in the Ancient World (pp. 193-215). Leiden: Brill.

Brulé, P. (2005). Comment dire le divin? In N. Belayche, P. Brulé, G. Freyburger, Y. Lehmann, L. Pernot, \& F. Prost (Eds.), Nommer les dieux. Théonymes, épithètes, épiclèses dans l'Antiquité (pp. 5-11). Turnhout: Presses Universitaires de Rennes; Brepols.

Bruneau, P. (1970). Recherches sur les cultes de Délos à l'époque hellénistique et à l'époque impériale. Paris: De Boccard.

Buck, C. D. $\left(1955=1988^{2}\right)$. The Greek Dialects. Revised Edition. Boston: Ginn and Company.

Bundy, E. L. (1962). Studia Pindarica. Berkeley - Los Angeles: University of California Press.

Burgess, J. (2006). Neoanalysis, Orality, and Intertextuality: An Examination of Homeric Motif Transference. Oral Tradition, 21(1), 148-189.

Burkert, W. (1975). Apellai und Apollon. Rheinisches Museum für Philologie, 19, 1-21.

Burkert, W. (1977). Griechische Religion der archaischen und klassischen Epoche. Stuttgart: Kohlhammer.

Burkert, W. (1984). Sacrificio - sacrilegio: il 'trickster' fondatore. Studi Storici, 4, 835-845.

Calame, C. (1998). Mûthos, logos et histoire. Usages du passé héroïque dans la rhétorique grecque. L'Homme, 38(147), 127-149.

Calame, C. (2008). Entre récit héroïque et poésie rituelle: le sujet poétique qui chant le mythe. In S. Parizet (Ed.), Mythe et littérature (Coll. Poétiques comparatistes; pp. 123-141). Paris: Lucie Éditions.

Calame, C. (2009). Apollo in Delphi and in Delos: Poetic Performances between Paean and Dithyramb. In L. Athanassaki, R. Martin, \& J. F. Miller (Eds.), Apolline politics and poetics: international symposium, Delphi 4-11 July 2003 (pp. 169-197). Athens: European Cultural Centre in Delphi. 
Calame, C. (2011). The Homeric Hymns as Poetic Offerings. Musical and Ritual Relationships with the Gods. In A. Faulkner (Ed.), The Homeric Hymns: Interpretative Essays (pp. 334-357). Oxford: University Press.

Calame, C. (2015). Qu'est-ce que la mythologie grecque? Paris: Gallimard.

Calame, C., \& Ellinger, P. (2016). Du récit au rituel par la forme esthétique. Poèmes, images et pragmatique rituelle en Grèce ancienne. Paris: Les Belles Lettres.

Cameron, A. (1995). Callimachus and his Critics. Princeton: University Press.

Cantilena, M. (1982). Ricerche sulla dizione epica, 1: Per uno studio della formularità degli Inni Omerici. Roma: Ed. dell'Ateneo.

Carrara, P. (1986). Stazio e i primordia di Tebe. Poetica e polemica nel prologo della Tebaide. Prometheus, 12, 146-158.

Càssola, F. $\left(1975=1991^{2}\right)$. Inni omerici. Milano: Mondadori.

Cerri, G. (1999). Parmenide di Elia, Poema sulla natura. Milano: Rizzoli.

Chailley, J. (1979). La musique grecque antique. Paris: Les Belles Lettres.

Chantraine, P. (1968). Dictionnaire etymologique de la langue grecque: histoire des mots. Paris: Klincksieck.

Chappell, M. (2011). The Homeric Hymn to Apollo. The Question of Unity. In A. Faulkner (Ed.), The Homeric Hymns. Interpretative Essays (pp. 59-81). Oxford: University Press.

Ciampa, S. (2006). I poeti ellenistici nei papiri ercolanesi di Filodemo. Cronache Ercolanesi, 36, 87-102.

Cingano, E. (1995). Pindaro. Le Pitiche (ed. B. Gentili, P. Angeli Bernardini, E. Cingano, \& P. Giannini). Milano: Mondadori.

Clark, M. E. (1986). Neoanalysis: A Bibliographical Review. The Classical World, 79, 379-394.

Colangelo, E. (2016). Unità e struttura dell'Inno omerico ad Apollo. In R. Di Donato (Ed.), Comincio a cantare. Contributi allo studio degli Inni omerici (pp. 2-13). Pisa: ETS.

Colangelo, E. (2017). Dinamiche di purificazione e abilitazione rituale nell'Inno omerico ad Apollo. In M. P. Castiglioni, M. Giuman, \& R. Carboni (Eds.), Hagnos, Miasma e Katharsis. Viaggio tra le categorie del puro e dell'impuro nell'immaginario del mondo antico, Atti del Convegno internazionale di Cagliari, Cittadella dei Musei, 4-6 maggio 2016, Otium (Archeologia e cultura del mondo antico, 2; pp. 1-29).

Colin, G. (1905). Le culte d'Apollon Pythien à Athènes. Paris: Fontemoing.

Colin, G. (1913). L'auteur du deuxième hymn musical de Delphes. Comptes Rendus de l'Academie des Inscriptions et Belles-Lettres, 8, 529-532.

Cook, A. B. (1928). Zeus: A Study in Ancient Religion (2 Vols.). Cambridge: University Press.

Crusius, O. (1894). Die delphischen Hymnen: Untersuchungen über Texte und Melodien. Göttingen: Dieterich.

Currie, B. (2005). Pindar and the Cult of Heroes. Oxford: University Press.

Currie, B. (2011). Perspectives on neoanalysis from the archaic hymns to Demeter. In $\varnothing$. Andersen, \& D. D. T. Haug (Eds.), Relative Chronology in Early Greek Epic Poetry (pp. 184-209). Cambridge: University Press.

D’Alessio, G. B. (1992). Pindaro, Peana VIIb (fr. 52h Sn.-M.). In A. H. S. El-Mosalamy et al. (Eds.), Proceedings of the XIX International Congress of Papyrology (Cairo 2-9 september 1989) (Vol. 1; pp. 353-373). Cairo: Ain Shams University, Center of Papyrological Studies.

D’Alessio, G. B. (1994). First-Person Problems in Pindar. Bulletin of the Institute of Classical Studies, 39, 117-140. 
D’Alessio, G. B. (1995). Una via lontana dal cammino degli uomini (Parm. frr. 1+6 D.K.; Pind. Ol. VI 22-27; Pae. VIIb 10-20). Studi italiani di filologia classica, 88, 143-181.

D’Alessio, G. B. (2000). Tra gli dèi ad Apollo, e tra gli uomini ad Echecrate. P. Louvre E7734+7733 (Pind. fr. dub. 333 S.-M.). In M. Cannatà Fera, \& S. Grandolini (Eds.), Poesia e religione in Grecia. Studi in onore di G. Aurelio Privitera (Vol. 1, pp. 233-262). Napoli: Edizioni Scientifiche Italiane.

D'Alessio, G. B. (2009). Pindar's First Hymn. The Theban 'Theogony' and the Birth of Apollo. In L. Athanassaki, R. Martin, \& J. F. Miller (Eds.), Apolline politics and poetics: international symposium, Delphi 4-11 July 2003 (pp. 129-147). Athens: European Cultural Centre in Delphi.

D’Alessio, G. B., \& Ferrari, F. (1988). Pindaro, Peana 6, 175-183: una ricostruzione. Studi Classici e Orientali, 38, 159-179.

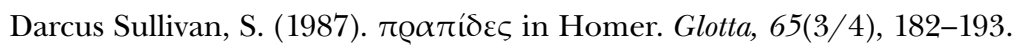

Darcus Sullivan, S. (1991). The Wider Meaning of Psyche in Pindar and Bacchylides. Studi italiani di filologia classica, 84, 163-183.

Darcus Sullivan, S. (1999). Sophocles' Use of Psychological Terminology: Old and New. Carleton (Ottawa): Carleton University Press.

Daux, G. (1936). Delphes au IIe et au Ier siècle: depuis l'abaissement de l'Etolie jusqu'à la paix romaine, 191-31 av. JC. Paris: De Boccard.

Defradas, J. (1954=1972²). Les thèmes de la propagande delphique. Paris: Klincksieck.

Detienne, M. (1977). Dionysos mis à mort. Paris: Gallimard.

Detienne, M. (1981). L'invention de la mythologie. Paris: Gallimard.

Detienne, M. (1988-1989). Conférence, in Compte-rendus de l'École Pratique des Hautes Études. Sections des sciences religieuses, 97, 267-270.

Detienne, M. $\left(1998=2009^{2}\right)$. Apollon le couteau à la main. Une approche expérimentale du polythéisme grec. Paris: Gallimard.

Detienne, M., \& Vernant, J.-P. (1979). La cuisine du sacrifice en pays grec. Paris: Gallimard.

Devine, A. M., \& Stephens, L. D. (1994). The Prosody of Greek Speech. New York - Oxford: University Press.

Di Donato, R. (1969). Problemi di tecnica formulare e poesia orale nell'epica greca arcaica. Annali della Scuola Normale Superiore di Pisa, 38(3/4), 243-294.

Digna, B. (2007). A Day in the Life of a Greek Sanctuary. In D. Ogden (Ed.), The Blackwell Companion to Greek Religion (pp. 163-177). Oxford: Blackwell.

Doutzaris, P. (1934). La rythmique dans la poésie et la musique des Grecs anciens. Revue des Études Grecques, 47, 297-345.

Dowden, K. (1996). Homer's sense of texte. Journal of Hellnic Studies, 116, 188-205.

Drozdek, A. (2007). Greek Philosophers as Theologians: the divine arche. Aldershot (UK) - Burlington (VT): Ashgate.

Duchemin, J. (1955). Pindare poète et prophète. Paris: Les Belles Lettres.

Edelstein, E. J., \& Edelstein, L. (1945). Asclepius: A Collection and Interpretation of the Testimonies. Baltimore: Johns Hopkins Press.

Esteban Santos, A. (1981). La estructura del Himno homérico a Apolo: un indicio importante de la división del poema. Cuadernos de Filología Clásica, 17, 193-214.

Fairbanks, A. (1900). A Study of the Greek Paean. Cornell: University Press.

Fantuzzi, M., \& Tsagalis, Ch. (2015). The Greek Epic Cycle and Its Ancient Reception. Cambridge: Cambridge University Press. 
Faraone, C. (1997). Hymn to Selene - Hecate - Artemis from a Greek Magical Handbook (PMG IV 2744-83). In M. Kiley et al. (Eds.), Prayer from Alexander to Constantine. A Critical Anthology (pp. 195-204). London - New York: Routledge.

Farrell, J. (2014). Looking for Empedocles in Latin Poetry: A Skeptical Approach. Dictynna, 11, $1-14$.

Faulkner, A. (2011). The Homeric Hymns. Interpretative Essays. Oxford: University Press.

Faulkner, A. (2015). The Silence of Zeus: Speech in the Homeric Hymns. In A. Faulkner, \& O. Hodkinson (Eds.), Hymnic Narrative and the Narratology of Greek Hymns (pp. 31-45). Leiden -Boston: Brill.

Faulkner, A., \& Hodkinson, O. (2015). Hymnic Narrative and the Narratology of Greek Hymns. Leiden - Boston: Brill.

Fayant, M. C. (2015). Les Hymnes orphiques. Paris: Les Belles Lettres.

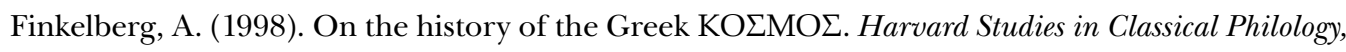
98, 103-136.

Foley, J. M. (1991). Immanent Art: From Structure to Meaning in Traditional Oral Epic. Bloomington, Ind.

Fontenrose, J. E. (1959). Python. A Study of Delphic Myth and its Origins. Berkeley - Los Angeles: University of California Press.

Fontenrose, J. E. (1960). The Cult and Myth of Pyrrhos at Delphi. University of California Publications in Classical Archeology, 4(3), 191-261.

Fontenrose, J. E. (1978). The Delphic Oracle. Its Responses and Operations, with a Catalogue of Responses. Berkeley: University of California Press.

Forderer, M. (1971). Anfang und Ende der abendländischen Lyrik. Untersuchungen zum Homerischen Apollonhymnos und zu Anise Koltz. Amsterdam: Grüner.

Frolíková, A. (1966). Ein Beitrag zur Frage der Zweiteilung des homerischen Apollonhymnos. Listy Filologické, 89, 1-8.

Furley, W. D., \& Bremer, J. M. (2001). Greek Hymns. Selected Cult Songs from the Archaic to the Hellenistic Period (2 Vols). Tübingen: Mohr Siebeck.

Führer, R. (1967). Formproblem - Untersuchungen zu den Reden in der frühgriechischen Lyrik. München: Beck.

Führer, R. (1977). Zur Meropis. Zeitschrift für Papyrologie und Epigraphik, 24, 42.

Gallavotti, C. (1980-1981). Da Stesicoro a Empedocle. Kokalos, 26-27, 413-433.

Gantz, T. (2004). Mythes de la Grèce archaïque. Paris: Belin.

Gentili, B. (1977). I cosiddetti dattilo-epitriti nella poesia orale pre-omerica, nelle iscrizioni arcaiche e nella lirica citarodica e corale da Stesicoro a Pindaro. Quaderni Urbinati di Cultura Classica, 26, 7-37.

Gérard-Rousseau, M. (1968). Les mentions religieuses dans les tablettes mycéniennes. Roma: Ed. dell'Ateneo.

Gignac, F. (1976). A Grammar of the Greek Papyri of the Roman and Byzantine Periods, I: Phonology. Milano: Ed. Cisalpino.

Giordano, M. (2010). Iliade I. L'ira - la peste. Roma: Carocci.

Giordano, M. (2011). Gli dèi nel De Musica. Quaderni Urbinati di Cultura Classica, 99(3), 59-72.

Giuseppetti, M. (2013). L'isola esile. Studi sull'Inno a Delo di Callimaco. Roma: Quasar. 
Graf, F. (1985). Nordische Kulte. Religionsgeschichtliche und epigraphische Untersuchungen zu den Kulten Chios, Erythra, Klazomenai und Phokaia. Roma: Schweizerisches Institut in Rom.

Grassien, C. (2005). Problèmes d'édition dans le corpus papyrologique des hymnes chrétiennes. Archiv für Papyrusforschung, 51, 253-279.

Graziosi, B., \& Haubold, J. (2005). Homer: the Resonance of Epic. Cambridge.

Grenfell, B. P., \& Hunt, A. S. (1922). The Oxyrhyncus Papyri (Vol. 15). London: Egyptian Exploration Society.

Guillon, P. (1963). Le bouclier d'Héraclès et l'histoire de la Grèce centrale dans la période de la première guerre sacrée. Aix-en-Provence: Éd. Ophrys.

Hagel, S. (2000). Modulation in altgriechischer Musik. Antike Melodien im Licht antiker Musiktheorie. Frankfurt: Peter Lang.

Hagel, S., \& Lynch, T. (2015). Music. In M. Bloomer (Ed.), A Companion to Ancient Education (pp. 401-412). Hoboken: Wiley-Blackwell.

Hainsworth, J. B. (1968). The Flexibility of the Homeric Formula. Oxford: Clarendon Press.

Hainsworth, J. B. (1978). Good and Bad Formulae. In B. C. Fenik (Ed.), Homer. Tradition and Invention (pp. 41-50). Leiden: Brill.

Hardie, A. (2000). Pindar's Theban' Cosmogony (The First Hymn). Bulletin of the Institute of Classical Studies, 44, 19-40.

Haspels, C. H. E. (1936). Attic black-figured Lekythoi (Ecole française d'Athènes; Travaux et mémoires, 4). Paris: De Boccard.

Henrichs, A. (1972). Toward a New Edition of Philodemus' Treatise On Piety. Greek, Roman, and Byzantine Studies, 13, 67-98.

Henrichs, A. (1983). Die Kekropidensage in P.Herc. 243. Cronache Ercolanesi, 13, 33-43.

Henrichs, A. (1984). The Sophists and Hellenistic Religion: Prodicus as the Spiritual Father of the Isis Aretalogies. Harvard Studies in Classical Philology, 88, 139-158.

Herda, A. (2008). Apollon Delphinios - Apollon Didymeus: zwei Gesichter eines milesischen Gottes und ihr Bezug zur Kolonisation Milets in archaischer Zeit. In R. Bol et al. (Eds.), Kult(ur) kontakte. Apollon in Milet / Didyma, Histria, Myus, Naukratis und auf Zypern. Akten der Table Ronde in Mainz vom 11.-12. März 2004 (pp. 13-85). Rahden (Westf.): Verlag Marie Leidorf.

Herington, J. (1985). Poetry into Drama: Early Tragedy and the Greek Poetic Tradition. Berkeley - Los Angeles: University of California Press.

Hopman-Govers, M. (2001). Le jeu des épithètes dans les Hymnes orphiques. Kernos, 14, 35-49.

Hunter, R. (2003). Theocritus. Encomium of Ptolemy Philadelphus. Berkeley - Los Angeles - London: University of California Press.

Hutchinson, G. O. H. (2001). Greek Lyric Poetry. A Commentary on Selected Larger Pieces. Oxford: University Press.

Huxley, G. (1975). Cretan Paiawones. Greek, Roman and Byzantine Studies, 15, 119-124.

Jameson, M. H. (1994). Theoxenia. In R. Hägg (Ed.), Ancient Greek cult practice from the epigraphical evidence. Proceedings of the Second International Seminar on ancient Greek cult, organized by the Swedish Institute at Athens, 22-24 November 1991 (ActaAth-8 ${ }^{\circ}$, 13; pp. 35-57). Stockholm: Diffusion Paul Aström.

Janko, R. (1982). Homer, Hesiod and the Hymns: Diachronic Development in Diction. Cambridge: Cambridge University Press. 
Janko, R. (1986). The Shield of Heracles and the legend of Cycnus. Classical Quarterly, 36, 38-59.

Janko, R. (1992). The Iliad: A Commentary. Volume IV: books 13-16. Cambridge: Cambridge University Press.

Jourdain-Annequin, C. (1988-1989). Être un Grec en Sicile: le mythe d'Héraklès. Kokalos, 34-35(1), 143-166.

Kannicht, R., \& Snell, B. (1981). Tragicorum Graecorum Fragmenta (TrGF), Vol. 2: Fragmenta adespota, testimonia volumini I addenda, indices ad volumina 1 et 2. Göttingen: Vandenhoeck \& Ruprecht.

Käppel, L. (1992). Paian. Studien zur Geschichte einer Gattung (Untersuchungen zur antiken Literatur und Geschichte, 37). Berlin - New York: de Gruyter.

Kenyon, F. G. (Ed.). (1907). Greek Papyri in the British Museum. London: Longmans.

Kerényi, K. (1976). Dionysos: Urbild des unzerstörbaren Lebens. München: Langen Müller.

Kingsley, P. (1995). Ancient Philosophy, Mystery and Magic. Empedocles and Pythagorean Tradition. Oxford: Clarendon Press.

Knatz, F. (1891). Empedoclea. In Schedae philologae Hermanno Usener a sodalibus Seminarii regii Bonnensis oblatae (pp. 1-9). Bonn: F. Cohen.

Koenen, L., \& Merkelbach, R. (1976). Apollodoros (ПEPI $\Theta E \Omega N)$, Epicharm und die Meropis. In A. E. Hanson (Ed.), Collectanea Papyrologica. Texts Published in Honor of H. C. Youtrie (Vol. 1; pp. 3-26). Bonn: Habelt.

Körte, A. (1932). Literarische Texte mit Ausschluß der christlichen. Archiv für Papyrusforschung, 10, 19-70.

Krevans, N. (1993). Fighting against Antimachus: the Lyde and the Aetia Reconsidered. In M. A. Harder, R. F. Regtuit, \& G. C. Wakker (Eds.), Callimachus (pp. 149-160). Groningen: Egbert Forsten.

Kullmann, W. (1984). Oral Poetry Theory and Neoanalysis in Homeric Research. Greek, Roman and Byzantine Studies, 25, 307-324.

Kyriazopoulos, A. (1988). Contributions des papyrus à l'étude des épithètes qualifiant Apollon dans Pindare. In B. G. Mandilaras et al. (Eds.), Proceedings of the XVIII International Congress of Papyrology (Vol. 1; pp. 365-368). Athens: Greek Papyrological Society.

Laurenti, R. (1995). Le proème à Apollon d'Empédocle dans les fragments d'Aristote. In A. Jannone et al. (Eds.), L'Aristote perdu (pp. 103-120). Roma - Atene: Comitato di studi sulla società contemporanea.

Lazzeri, M. (1999). Note al Papiro di Lille di Stesicoro. Bollettino dei Classici, ser. 3, 20, 19-35.

Le Guen, B. (2001). Les associations des Technites dionysiaques à l'époque hellénistique (2 Vols.). Paris: De Boccard.

Lefkowitz, M. R. (1991). First-Person Fictions. Pindar's Poetic 'I'. Oxford: Clarendon Press.

Lloyd-Jones, H. (1984). The Meropis (SH 903a). In M. Gigante (Ed.), Atti del XVII Congresso Internazionale di Papirologia (Vol. 1; pp. 141-150). Napoli: Centro Internazionale per lo studio dei papyri Ercolanesi.

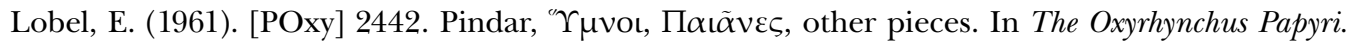
Part XXVI (pp. 31-78). London: Egypt Exploration Society.

Maas, M., \& MacIntosh Snyder, J. (1989). Stringed Instruments of Ancient Greece. New Haven - London: Yale University Press.

MacGillivray, J. A., \& Driessen, J. \& Sackett, L. H. (2000). The Palaikastro Kouros: a Minoan chryselephantine statuette and its Aegean Bronze Age context. London: British School at Athens. 
Maresch, K. (1991). Kölner Papyri (Vol. VII). Opladen: Westdeutscher Verlag.

Martin, R. (2005). Epic as genre. In J. M. Foley (Ed.), A Companion to Ancient Epic (pp. 9-19). Oxford: Blackwell.

Martin, R. (2013). Review a Montanari, F., Rengakos, A., \& Tsagalis C. C., 2012. Bryn Mawr Classical Review 2009.09.21 (retrieved 17.10.2017 from http://bmcr.brynmawr.edu/2013/2013-09-21.html).

Massa, F. (2006-2007). Dioniso e Apollo dal teatro attico alla cultura imperiale: tratti salienti di un complesso quadro documentario. Mythos, 1, 77-92.

Matthews, V. J. (1996). Antimachus of Colophon. Leiden - New York - Köln: Brill.

Méautis, G. (1962). Pindare le Dorien. Neuchatel: Albin Michel.

Meliadò, C. (2004). P. Chic. 1061 = P. Goodspeed 2. Proposte di lettura e interpretazione. Zeitschrift für Papyrologie und Epigraphik, 150, 49-58.

Meliadò, C. (2007). Da Cos a Delo: nuovi scenari mitologici in P. Lit. Goodspeed 2. In J. Frösén et al. (Eds.), Proceedings of the 24th International Congress of Papyrology (Helsinki 1-7 August 2004) (Vol. 2; pp. 729-733). Helsinki: Societas Scientiarum Fennica.

Meliadò, C. (2008). E cantando danzerò. Messina: Orione.

Micheli, M. (1995). La statua di Apollo Delios, opera di Tektaios e Angelion. Prospettiva, 78, 2-21.

Mikalson, J. (1998). Religion in Hellenistic Athens. Berkeley: University of California Press.

Mineur, W. H. (1984). Callimachus. Hymn to Delos. Leiden: Brill.

Moens, W. P. (1930). De twee delphische Hymnen met muzieknoten. Purmerend: J. Muusses.

Monbrun, P. (2007). Les voix d'Apollon. L'arc, la lyre et les oracles. Rennes: Presses Universitaires.

Montanari, F., Rengakos, A., \& Tsagalis C. C. (2012). Homeric Contexts. Neoanalysis and the Interpretation of Oral Poetry (Trends in Classics, Supplementary Volumes, 12). Berlin - Boston: de Gruyter.

Morand, A.-F. (2005). Oppositions et jeux phoniques. Le sens et le son dans les Hymnes orphiques. In A. Kolde et al. (Eds.), «Koruphaiôi andri». Mélanges offerts à André Hurst (pp. 223-233). Genève: Droz.

Nachtergael, G. (1977). Les Galates en Grèce et les Sôtéria de Delphes: Recherches d'histoire et d'épigraphie hellénistiques (Académie Belgique, Mémoires de la Classe des Lettres, 63/1). Bruxelles: Academia Belgica.

Nagy, G. (1979). The Best of the Achaeans. Baltimore: Johns Hopkins University Press.

Nagy, G. (1981). An Evolutionary Model for the Text Fixation of Homeric Epos. In J. M. Foley (Ed.), Oral Traditional Literature: A Festschrift for Albert Bates Lord (pp. 390-393). Ohio: Slavica Publishers.

Nagy, G. (2011). The Earliest Phases in the Reception of the Homeric Hymns. In A. Faulkner (Ed.), The Homeric Hymns. Interpretative Essays (pp. 280-333). Oxford: University Press.

Nisetich, F. (2001). The Poems of Callimachus. With introduction, notes, and glossary. Oxford: University Press.

Olson, S. D. (2012). The "Homeric Hymn to Aphrodite" and Related Texts: Text, Translation and Commentary. Berlin: de Gruyter.

Page, D. L. (1955). Sappho and Alcaeus. An introduction to the study of ancient Lesbian poetry. Oxford: Clarendon Press.

Page, D. L. (1967²=1962). Poetae Melici Graeci. Oxford: Clarendon Press.

Palmer, J. (2009). Parmenides and Presocratic Philosophy. Oxford: University Press.

Pardini, A. (1991). La ripartizone in libri dell'opera di Alceo. Rivista di filologia e di istruzione classica, 120, 257-284. 
Parke, H. W., \& Wormell, D. E. W. (1956). The Delphic Oracle (2 Vols.). Oxford: Blackwell.

Parker, R. (1996). Athenian Religion: A History. Oxford: Clarendon Press.

Parker, R. (2003). The Problem of the Greek Cult Epithet. Opuscula Atheniensia, 28, 173-183.

Parker, R. (2005). Greek Polytheism at Athens. Oxford: University Press.

Parker, R. (2011). On Greek Religion. Ithaca, NY - Londres: Cornell University Press.

Parry, M. (1928). L'épithète traditionnelle dans Homère. Essai sur un problème de style homérique. Paris: Les Belles Lettres.

Parsons, P. J. (1977). The Lille Stesichorus. Zeitschrift für Papyrologie und Epigraphik, 26, 7-36.

Pavese, C. O. (1993). Il coro nel sesto Peana di Pindaro. In R. Pretagostini (Ed.), Tradizione e innovazione nella cultura greca da Omero all'età ellenistica. Scritti in onore di Bruno Gentili (pp. 469-479). Roma: Gruppo Editoriale Internazionale.

PEG = Bernabé, A. (2004). Poetae epici Graeci. Testimonia et Fragmenta. Pars II. Orphicorum et Orphicis similium testimonia et fragmenta. Fasciculus I. Monachii - Lipsiae: In Aedibus K. G. Saur.

Perlman, P. J. (1995). Invocatio and Imprecatio: the Hymn to the Greatest Kouros from Palaikastro and the Oath in Ancient Crete. Journal of Hellenic Studies, 115, 161-167.

Petrovic, I. (2012). Callimachus' Hymn to Apollo and Greek Metrical Sacred Regulations. In M. A. Harder, R. F. Regtuit, \& G. C. Wakker (Eds.), Hellenistica Groningana. Gods and Religion in Hellenistic Poetry. Proceedings of the Ninth Groningen Workshop on Hellenistic Poetry (pp. 281-306). Leuven: Peeters.

Pfeiffer, R. (1949-1953). Callimachus (Vol. 2). Oxford: University Press.

Picot, J.-C. (2000). L'Empédocle magique de P. Kingsley. Révue de Philosophie Ancienne, 18(1), 25-86.

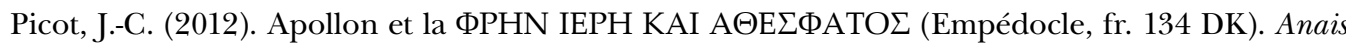
de filosofia clássica, 6(11), 1-31.

Pingiatoglu, S. (1981). Eileithyia. Würzburg: Königshausen \& Neumann.

Pironti, G., \& Pirenne Delforge, V. (2016). L’Héra de Zeus. Ennemie intime, épouse définitive. Paris: Les Belles Lettres.

Powell, J. U. (1918). Fragments of Greek Poetry from Papyri in the Library of the University of Chicago. The Journal of Philology, 34, 106-128.

Powell, J. U. (1925). Collectanea Alexandrina. Reliquiae Minores Poetarum Gravorum Aetatis Ptolemaicae, 323-146 A.C. Epicorum, Elegiacorum, Lyricorum, Ethicorum. Cum Epimetris et Indice Nominum. Oxford: Clarendon Press.

Pöhlmann, E., \& West, M. (2001). Documents of Ancient Greek Music. Oxford: Clarendon Press.

Preisendanz, K., Heitsch, E., \& Henrichs, A. (1973-1974²). Papyri Graecae Magicae. Die griechischen Zauberpapyri. Stuttgart: Teubner.

Primavesi, O. (2007). Empédocle: divinité physique et mythe allégorique. Philosophie antique, 7, 51-89. Primavesi, O. (2011). Empedokles-Texte und Übersetzungen. In J. Mansfeld, \& O. Primavesi (Eds.), Die Vorsokratiker (pp. 421-563). Stuttgart: Reclam.

Radt, S. L. (1958). Pindars zweiter und sechster Paian. Amsterdam: Hakkert.

Reed, D. J. (1996). Antimachus on Adonis? Hermes, 124, 381-383.

Reinach, T. (1893). La Musique des hymnes de Delphes. Bulletin de correspondance hellénique, 17, 584-610.

Reinach, T. (1909-1913). Hymnes avec notes musicales. In M. G. Colin (Ed.), Fouilles de Delphes (Vol. III, 2; pp. 147-169). Paris: De Boccard. 
Richardson, N. (1985). Pindar and later literary criticism in Antiquity. Papers of the Liverpool Latin Seminar, 5, 383-401.

Richardson, N. (2010). Three Homeric Hymns: to Apollo, Hermes and Aphrodite: Hymns 3, 4 and 5. Cambridge: University Press.

Roscher, W. H. (1884-1937). Ausführliches Lexikon der griechischen und römischen Mythologie (6 Vols.). Leipzig - Berlin: Olms; Georg.

Rousset, D. (2002). Le territoire de Delphes et la terre d'Apollon. Paris: De Boccard.

Ruck, C. A. P. (1976). Duality and the Madness of Herakles. Arethusa, 9, 53-75.

Rudhardt, J. (1991). Quelques réflexions sur les Hymnes orphiques. In Ph. Borgeaud (Ed.), Orphisme et Orphée (pp. 263-283). Genève: Droz.

Rutherford, I. C. (1988). Pindar on the birth of Apollo. Classical Quarterly, 38, 65-75.

Rutherford, I. C. (1997). For the Aeginetans to Aiakos a Prosodion: An Unnoticed Title at Pindar, Paean 6, 123, and its Significance for the Poem. Zeitschrift für Papyrologie und Epigraphik, 118, 1-21.

Rutherford, I. C. (2001). Pindar's Paians: A Reading of the Fragments with a Survey of the Genre. Oxford: University Press.

Rutherford, I. C. (2009). The Koan-Delian ritual complex. Apollo and Theoria in a sacred law from Kos. In L. Athanassaki, R. Martin, \& J. F. Miller (Eds.), Apolline politics and poetics: international symposium, Delphi 4-11 July 2003. Hellenic Ministry of Culture: European Cultural Centre at Delphi (pp. 655-687). Athens: European Cultural Centre in Delphi.

Sbardella, L. (2000). Filita. Frammenti dell'opera poetica. Roma: Quasar.

Sbardella, L. (2012). Cucitori di canti. Studi sulla tradizione epico-rapsodica greca e i suoi itinerari nel VI secolo a.C. Roma: Quasar.

Schachter, L. (1981). Cults of Boiotia (BICS Supplement, 38; 2 Vols.). London: Institute of Classical Studies.

Scheid, J., \& Svenbro, J. (1996). The Craft of Zeus. Myths of Weaving and Fabric. Cambridge, Mass.: Harvard Unviersity Press.

Schmidt, V. (1975). Zu Pindar. Glotta, 53, 36-43.

Schober, A. (1923). Philodemi de pietate. Pars prior. Diss. Königsberg.

Schröder, S. (1999). Zwei Überlegungen zu den Liedern vom Athenerschatzhaus in Delphi. Zeitschrift für Papyrologie und Epigraphik, 128, 65-75.

Schroeder, O. (1923). Pindari carmina cum fragmentis selectis. Leipzig: Teubner.

Schubert, P. (1996). Les papyrus de Genève. Textes littéraires et documentaires, 3: nos. 118-146. Genève: Bibliothèque publique et universitaire.

Schwyzer, E. (1939). Griechische Grammatik auf der Grandlage von Karl Brugmanns griechischer Grammatik (Vol. I; Handbuch der Altertumswissenschaft, 2.1). München: Beck.

Schwyzer, E., \& Debrunner, A. $\left(1950=1966^{3}\right)$. Griechischer Grammatik Zweiter Band: Syntax und syntaktische Stilistik. München: C. H. Beck.

Scott, M. (2014). Delphi: A History of the Center of the Ancient World. Princeton: University Press.

Seaford, R. (2005). Mystic Light in Aeschylus' Bassarai. Classical Quarterly, 55(2), 602-606.

Serrao, G. (1979). La struttura della Lide di Antimaco e la critica callimachea. Quaderni Urbinati di Cultura Classica, 32, 91-98.

Sherwin-White, S. M. (1978). Ancient Cos. An Historical Study from the Dorian Settlement to the Imperial Period. Göttingen: Vandenhoeck and Ruprecht. 
Slater, W. J. (1969). Lexicon to Pindar. Berlin: De Gruyter.

Snell, B. (1975 $)$. Pindars Hymnus auf Zeus. In B. Snell (Ed.), Die Entdeckung des Geistes. Studien zur Entstehung des europäischen Denkens bei den Griechen (reprinted; pp. 82-94). Göttingen: Vandenhoeck and Ruprecht.

Snell, B., \& Maehler, H. (1971). Pindari Carmina cum fragmentis. Leipzig: Teubner.

Sokolowski, F. (1969). Lois sacrées des cités grecques. Paris: De Boccard.

Solmsen, F. (1980). Empedocles' Hymn to Apollo. Phronesis, 25, 219-227.

Solomon, J. (2000). Ptolemy 'Harmonics': Translation and Commentary. Boston - Leiden - Köln: Brill. Sourvinou-Inwood, C. (1979). The myth of the first temples at Delphi. Classical Quarterly, 29, 231-251. Stefos, A. (1975). Apollon dans Pindare. Diss. Athènes.

Stratiki, K. A. (2013). Le 'Dionysoi' de Patras: le mythe et le culte de Dionysos dans la Periégèse de Pausanias. In A. Bernabé, M. Herrero, A. J. Jiménez, \& R. Martín-Hernández (Eds.), Redefining Dionysos (pp. 401-414). Berlin: De Gruyter.

Strauss Clay, J. $\left(1989=2006^{2}\right)$. The Politics of Olympus: Form and Meaning in the Major Homeric Hymns. Princeton: University Press.

Suárez de la Torre, E. (1993). Pindaro y la religion griega. Cuadernos de Filología Clásica (G), 3, 67-97. Suárez de la Torre, E. (2002). Yambógrafos griegos. Madrid: Editorial Gredos.

Suárez de la Torre, E. (2007). Neoptolemos at Delphi. Kernos, 10, 153-176.

Suárez de la Torre, E. (2009). La divinazione nei Papiri Magici Greci. In M. Monaca (Ed.), Problemi di storia religiosa del mondo tardo-antico. Tra mantica e magia (pp. 13-52). Cosenza: Giordano.

Tarán, L. (1981). Speusippus of Athens. A Critical Study with a Collection of Related Texts and Commentary. Leiden: Brill.

Teffeteller, A. (2001). The Chariot Rite at Onchestos: Homeric Hymn to Apollo 229-238. Journal of Hellenic Studies, 121, 159-166.

Tsitsibakou-Vasalos, E. (1985). Stesichorus and his Poetry. Diss. Chicago.

Van der Berg, R. M. (2001). Proclus' Hymns. Essays, Translations, Commentary. Leiden - Boston Köln: Brill.

Vergados, A. (2013). The Homeric Hymn to Hermes. Introduction, Text and Commentary. Berlin - Boston: De Grutyer.

Vernant, J.-P. (1962). Les Origines de la pensée grecque. Paris: PUF.

Vernant, J.-P. (1974). Mythe et société dans la Grèce ancienne. Paris: Maspero.

Veyne, P. (2000). Inviter les dieux, sacrifier, banqueter. Quelques nuances de la religiosité gréco-romaine. Annales, Histoire, Sciences Sociales, 55, 3-42.

Virgilio, B. (1993). Gli Attalidi di Pergamo. Fama, Eredità, Memoria (Studi ellenistici, 5; Biblioteca di studi antichi, 70). Pisa - Roma: Giardini.

Vitek, T. (2010). Le 'Sphairos' d'Empédocle et son substrat mythologique. Elenchos, 3(1), 43-44.

Vitelli, G. (1913). Frammenti di Peani di Pindaro (Editio princeps di PSI 147). In Papiri greci e latini (Vol. 2; pp. 73-79). Firenze: Ariani.

Wackernagel, J. (1943). Graeca. Philologus, 95, 177-192.

Wade-Gery, H. T. (1936). Kynaithos. In C. Bailey et al. (Eds.), Greek Poetry and Life: Essays Presented to Gilbert Murray on his Seventieth Birthday (pp. 56-78). Oxford: Clarendon Press.

Wallensten, J. (2008). Personal protection and tailor-made deities: the use of individual epithets. Kernos, 21, 81-95. 
Weber, G. (1993). Dichtung und höfische Gesellschaft. Die Rezeption von Zeitgeschichte am Hof der ersten drei Ptolemäer. Stuttgart: Franz Steiner Verlag.

Weil, H. (1893). Nouveaux fragments d'hymnes accompagnés de notes de musique. Bulletin de Correspondance Hellénique, 17, 569-583.

West, M. L. (1975). Cynaethus' Hymn to Apollon. The Classical Quarterly, 25(2), 161-170.

West, M. L. (1990). Studies in Aeschylus. Stuttgart: Teubner.

West, M. L. (1992). Analecta Musica. Zeitschrift für Papyrologie und Epigraphik, 92, 1-54.

West, M. L. (1992b). Ancient Greek Music. Oxford: Clarendon Press.

West, M. L. (1995). The Date of Iliad. MH, 52(4), 203-219.

Wilamowitz-Moellendorff, U. von (1908). Pindars siebentes nemeisches Gedicht. Berlin: SPAW.

Williams, F. (1978). Callimachus' Hymn to Apollo: A Commentary. Oxford: Clarendon Press.

Wyss, B. (1935). Antimachi Colophonii reliquiae. Berlin: Weidmann.

Zaborowski, R. (2008). Sur les sentiments chez les Présocratiques: contribution psychologique à la philosphie des sentiments. Varsovie: Stakroos.

Zuntz, G. (2005). Griechische philosophische Hymnen (ed. H. Cancik, \& L. Käppel; Studien und Texte zu Antike und Christentum, 35). Tübingen: Mohr Siebeck.

Eleonora Colangelo / eleonora.colangelo@univ-paris-diderot.fr

Centre AnHiMA (Anthropologie et Histoire des Mondes Antiques)

2, rue Vivienne, 75002 Paris, France

Paris Diderot University, Faculté de Géographie, Histoire, Economie et Sociétés

5 rue Thomas Mann, 75013 Paris, France

University of Pisa, Departimento di Filologia Letteratura Linguistica

Palazzo Matteucci, Piazza Torricelli 2, 56126 Pisa, Italy 